\title{
BRENDA: A DYNAMIC SIMULATOR FOR A SODIUM-COOLED FAST REACTOR POWER PLANT
}

\author{
D. L. Hetrick and G. W. Sowers \\ University of Arizona
}

\author{
Prepared for \\ U. S. Nuclear Regulatory Commission
}




\section{DISCLAIMER}

This report was prepared as an account of work sponsored by an agency of the United States Government. Neither the United States Government nor any agency Thereof, nor any of their employees, makes any warranty, express or implied, or assumes any legal liability or responsibility for the accuracy, completeness, or usefulness of any information, apparatus, product, or process disclosed, or represents that its use would not infringe privately owned rights. Reference herein to any specific commercial product, process, or service by trade name, trademark, manufacturer, or otherwise does not necessarily constitute or imply its endorsement, recommendation, or favoring by the United States Government or any agency thereof. The views and opinions of authors expressed herein do not necessarily state or reflect those of the United States Government or any agency thereof. 


\section{DISCLAIMER}

Portions of this document may be illegible in electronic image products. Images are produced from the best available original document. 


\section{NOTICE}

This report was prepared as an account of work sponsored by the United States Government. Neither the United States nor the United States Nuclear Regulatory Commission, nor any of their employees, nor any of their contractors, subcontractors, or their employees, makes any warranty, express or implied, nor assumes any legal liability or responsibility for the accuracy, completeness or usefulness of any information, apparatus, product or process disclosed, nor represents that its use would not infringe privately owned rights.

Available from

National Technical Information Service Springfield, Virginia 22161

Price: Printed Copy $\$ 6.00$; Microfiche $\$ 3.00$

The price of this document for requesters outside of the North American Continent can be obtained from the National Technical Information Service. 


\section{BRENDA: A DYNAMIC SIMULATOR FOR A SODIUM-COOLED FAST REACTOR POWER PLANT}

D. L. Hetrick and G. W. Sowers

Manuscript Completed: June 1978

Date Published: July 1978

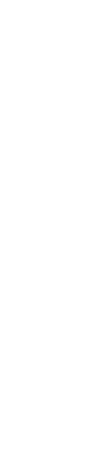


ABSTRACT

This report is a users' manual for one version of BRENDA (Breeder Reactor Nuclear Dynamic Analysis), which is a digital program for simulating the dynamic behavior of a sodium-cooled fast reactor power plant. This version, which contains 57 differential equations, represents a simplified model of the Clinch River Breeder Reactor Project (CRBRP).

BRENDA is an input deck for DARE P (Differential Analyzer Replacement, Portable), which is a continuous-system simulation language developed at the University of Arizona. DARE P is available from the University at nominal cost, and is operational on several types of computers.

This report contains brief descriptions of DARE $P$ and BRENDA, instructions for using BRENDA in conjunction with DARE $P$, and some sample output. A list of variable names and a listing for BRENDA are included as appendices.

This work was performed at the University of Arizona with support from the U. S. Nuclear Regulatory Commission under Contract No. $\operatorname{AT}(49-24)-0250$ 
TABLE OF CONTENTS

Page

INTRODUCTION . . . . . . . . . . . . . . . . . . 1

DARE P............................. . . . . . . 3

BRENDA . . . . . . . . . . . . . . . . . . . . 7

HOW TO RUN PLANT TRANSIENTS. . . . . . . . . . . . . . 12

ISOLATING PORTIONS OF THE PLANT. . . . . . . . . . . . . . . 15

SAMPLE OUTPUT. . . . . . . . . . . . . . . . . . . . . . 18

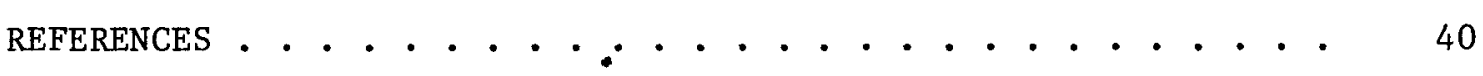

APPENDICES

A. Glossary of Variable Names Used in BRENDA . . . . . . . 41

B. BRENDA, a DARE P Input File. . . . . . . . . . . . 54

C. Some Comments on Computer Graphics . . . . . . . . . . 81

POSTSCRIPT . . . . . . . . . . . . . . . . . . 82 


\section{INTRODUCTION}

A digital simulator of a liquid metal fast breeder reactor (LMFBR) power plant has been developed at the University of Arizona under the sponsorship of the Division of Reactor Safety Research of the United States Nuclear Regulatory Commission. Versions of the model that have been tested contain up to 72 differential equations representing the reactor, the heat exchangers, the steam generator, the turbo-generator system, and various controllers. Special transport delay operators have been developed that employ variable time delays which are computed from varying flow rates in pipes.

The model has been used to study dynamic behavior and operational safety of a prototype demonstration plant, the Clinch River Breeder Reactor Plant design (Jacobi, 1974). Sensitivity to model variations and to values of parameters has been extensively investigated. Examples of transients include reactivity insertions, pressure, temperature, flow, and electric load variations, and reactor scram followed by pump coastdown. Transients of the latter type are studied with the aid of modules (not included in this version) that represent natural convection and the auxiliary heat removal system of the plant.

Details of the model are given in the dissertation by Shinaishin (1976) and in the paper by Hetrick, Shinaishin, and Wait (1978). The version described here has a number of limitations; e.g., 
a single heat transport loop (a composite of three loops), and a very elementary model of the turbine-feedwater loop.

BRENDA is an input deck for the DARE P (Differential Analyzer Replacement, Portable) continuous-system simulation language developed at the University of Arizona by Professors Granino Korn and John Wait (Korn and Wait, 1978). DARE $P$ is operational on several types of computers including CDC, IBM, and UNIVAC systems. We have used both a CDC-6400 and a CDC CYBER-175, the latter since December 1977.

The DARE P system contains a number of integration rules as options, including variable-step implicit methods that can accommodate a high degree of stiffness. Most of our results have been obtained using a variable-step Runge-Kutta method, with frequent check cases using the EPISODE implicit method (Byrne and Hindmarsh, 1975). The system can be dimensioned to accommodate 200 differential equations. Output options, controlled by simple one-line commands, include numerical listings, time plots, and state-plane plots.

The scope of this report is limited to brief descriptions of DARE $P$ and BRENDA, instructions for using one version of BRENDA in conjunction with DARE $P$, a listing of BRENDA, and some sample output. Inquiries about DARE $P$ may be directed to Dr. John Wait, Department of Electrical Engineering, University of Arizona. This version of BRENDA will be submitted to the Argonne Code Center; meanwhile, inquiries may be directed to Dr. David Hetrick, Department of Nuclear Engineering, University of Arizona. 
DARE $P$

DARE P (Differential Analyzer Replacement, Portable) is an equation-oriented continuous-system simulation language developed at the University of Arizona with support from the National Science Foundation (Korn and Wait, 1978). It can be dimensioned to solve an initial-value problem consisting of 200 simultaneous first-order nonlinear differential equations (state equations).

A typical input deck for DARE $P$ consists of the following:

1. A method block, which for the integration schemes built into DARE $P$ consists of one card of the form \$Mm (starting in co1. 2); $m=1$ selects a variable-step Runge-Kutta-Merson method, $\mathrm{m}=3$ selects Gear's method (Gear, 1971), $\mathrm{m}=11$ selects EPISODE (Byrne and Hindmarsh, 1975), etc. Other methods are also included, or the user may incorporate his own method. The default method is \$MI.

2. A derivative block, introduced by the card $\$ D 1$, and containing the differential equations, any algebraic equations, values of fixed parameters, procedure blocks for logical operations, . comments indicated by asterisks, and STORE commands for identifying the desired output variables. Formulas and statements are standard FORTRAN, except that, for example, the differential equation for the state variable $X$ is written as

$$
\mathrm{X} .=\mathrm{F}
$$


where $F$ is a FORTRAN expression. The translator converts this equation into whatever representation of a differential equation is appropriate for each integration method.

3. Function blocks, introduced by the card $\$ F$, which play the role of subroutines that may be called in the derivative block.

4. Tables, signified by cards of the form $\$ T m$ where $m$ is an integer. Tables may be one-dimensional or two-dimensional. Interpolated values are called by statements in the derivative block.

5. A logic block (\$L) for controlling simulator runs and storing desired solutions. Its absence (default) signifies a single run.

6. An END card (cols. 1-3).

7. An initial-condition block containing run-control parameters, initial values of state variables (if non-zero), and any parameter values that were not stated in the derivative block. The only card that must appear is the definition of the total simulation time TMAX. Other cards override various default values, such as NPOINT (number of listed output time points; default 101), EMAX (maximum relative error permitted in variable-step methods), etc.

8. An END card.

9. Output commands such as

LIST $\mathrm{X} 1, \mathrm{X} 2$

PLOT X3 
which generate listings and line-printer plots. The plots are automatically scaled by DARE P. The option of generating CALCOMP plots is included.

10. An END card.

Details of these and other features are discussed in the book by Korn and Wait (1978).

DARE P involves four overlays or memory loads; in our local CYBER 175 installation, a DARE $\mathrm{P}$ job is controlled by a procedural file which is called by a single control card. In other systems, the overlays must be sequenced by the local job control language. The source program for DARE $P$ is in standard ANSI FORTRAN IV.

In operation, DARE $P$ translates and sorts the equations in the derivative block. The problem will not run if an implicit algebraic loop is encountered at this stage. For proper operation of the sorting process, each procedure block contains a provision for stating in its initial line which variable is defined by, and which variables are needed by, the statements contained in that block.

After sorting, DARE $P$ calls the translated form of the derivative block at each time step, updates the state variables, and advances the time step. In a variable-step method, the step size may increase, decrease, or remain fixed depending on an appropriate error criterion. In addition, a special computation is made at each time point for which printed output is required.

Whenever the error criterion calls for a decreasing time step, the latest results are discarded and recomputed at one half of the 
time interval. The user must be careful that any switches that might be activated by discarded values of variables are reset before recomputation. Otherwise, "spurious excursions" such as unwanted activation of controllers may be introduced.

A simulator for a particular physical system (such as BRENDA) is comprised of an input deck for DARE P. Such an input deck can be quite large and unwieldy (many hundreds of cards). It is most conveniently maintained on a disk file and edited interactively. Alternatively, on CDC systems, it could be stored as a permanent file and modified by UPDATE corrections. The sample listing provided as an appendix to this report was printed from a DEC-10 disk file in which each line is a card image.

The DARE $P$ program itself is maintained as a permanent file in our CYBER-175, and BRENDA, preceded by an appropriate job card and control card, is sent from the DEC-10 over a computer link to the CYBER. Output may be printed, or returned as a data file on the DEC-10.

DARE $P$ may be obtained on tape at nominal cost from Dr. John Wait, Department of Electrical Engineering, University of Arizona. 
BRENDA

BRENDA (Breeder Reactor Nuclear Dynamic Analysis) is a lumpedparameter dynamic simulator for studying the approximate response of a sodium-cooled fast reactor power plant to various hypothetical perturbations. The version described here is a model for the CRBRP (Clinch River Breeder Reactor Plant) design (Jacobi, 1974) in which the three-1oop heat-transport system is represented as a single composite 1oop. See Fig. 1 .

The purpose of BRENDA is to permit inexpensive dynamic simulations for (1) scoping studies, (2) identification of key dynamic variables, (3) identification of cases for more detailed study, (4) sensitivity studies, and (5) ballpark verification. Typical transients that may be studied include reactivity insertions, flow perturbations, and electric load variations. Although many nonlinear phenomena are included in the model, the presence of many simplifying assumptions precludes the use of the model for predicting extreme off-normal behavior.

Details of the model are described in the dissertation by Shinaishin (1976) and in the paper by Hetrick, Shinaishin, and Wait (1978). This version contains some major changes from the original version, as discussed in the following.

The original version of the transport delay operator PDELAY, which is designed to permit the use of variable time delays calculated from variable flow rates in pipes, contained an option which included 


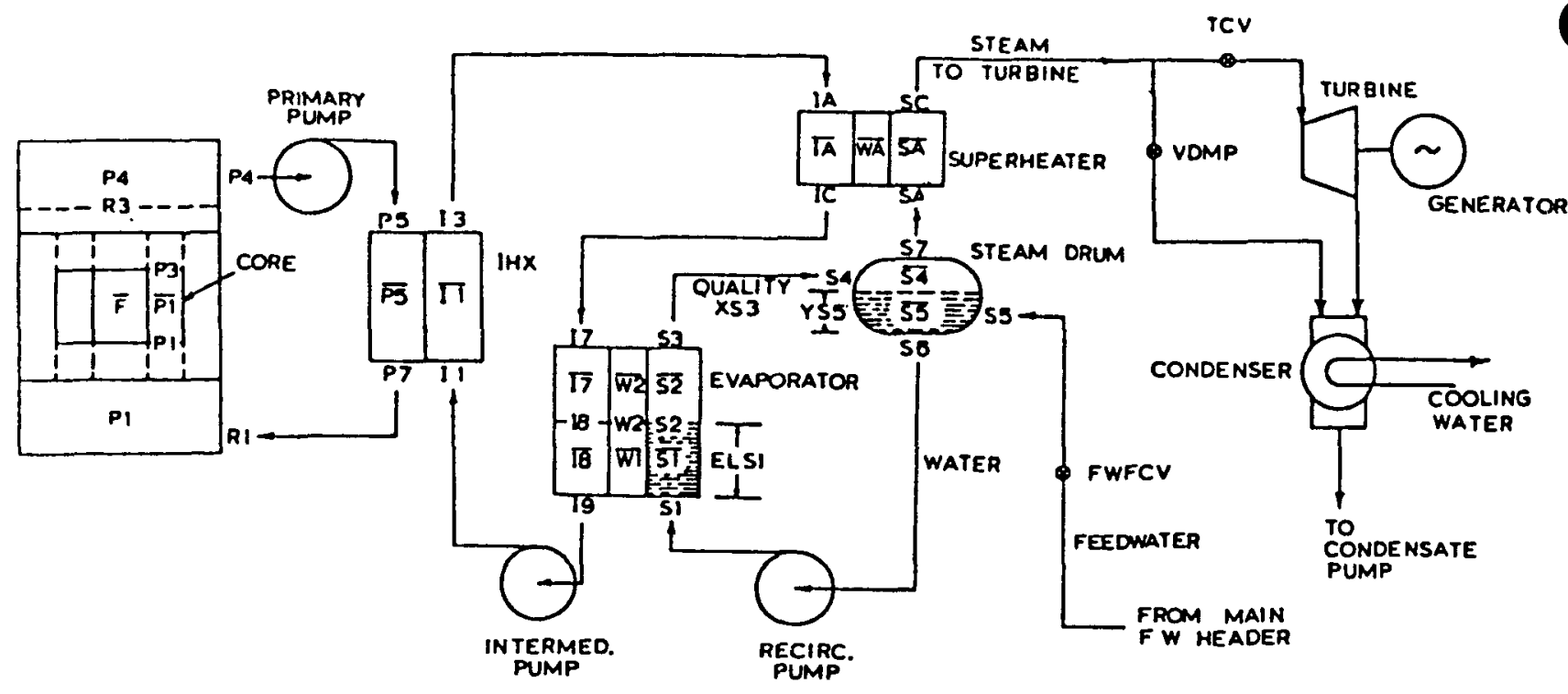

Temperature

$\overline{\mathrm{T}}_{\mathrm{AF}}$

$T_{\text {IC }}\left(855^{\circ} \%\right)$

$\mathrm{T}_{\mathrm{I} 3}\left(936^{\circ} \mathrm{F}\right)$

T IB

$\mathrm{T}_{\text {I9 }}\left(651^{\circ} \mathrm{F}\right)$

$\mathrm{T}_{\mathrm{Pl}}\left(730^{\circ} \mathrm{F}\right)$

$T_{P 3}$

$\mathrm{T}_{\mathrm{P4}}\left(995^{\circ} \mathrm{F}\right)$

$\mathrm{T}_{\mathrm{P} 7}\left(730^{\circ} \mathrm{F}\right)$

$\mathrm{T}_{\text {R3 }}$

$\mathrm{T}_{\mathrm{SC}}\left(905^{\circ} \mathrm{F}\right)$

$\overline{\mathrm{T}}_{\text {WA }}$

$\bar{T}_{W 1}$

$\bar{T}_{W 2}$
Flow

Primary (11518 1bm/sec)

Intermed (10650 $1 \mathrm{bm} / \mathrm{sec})$

RIser (1856 1bm/sec)

Downcomer (1856 1bm/sec)

S.H. -Turbine (928 1bm/ sec)

Feedwater (928 $1 \mathrm{bm} / \mathrm{sec}$ )

Pressure

$\overline{\mathrm{P}}_{\mathrm{S4}}(1765 \mathrm{ps1})$

$\overline{\mathrm{P}}_{\mathrm{S} 2}(1830 \mathrm{psi})$

$\underline{\text { Pump Speed }}$

Primary (1170 RPM)

Intermed.(1006 RPM)
Mas8

${ }^{\text {S4 }}$

$\mathrm{M}_{\mathrm{S} 5}$

Water HeIght

$\mathrm{I}_{\mathrm{S1}}$

Water Enthalpy

$\mathrm{H}_{\mathrm{S6}}\left(\mathrm{Q} 544^{\circ} \mathrm{F}\right)$

Turbine Varlables

Power Angle

Turbine Speed (60 RPS)

Turbine Power(380 MW)

Delayed Neutrons

S1x Groups

Fig. 1. Schematic Dlagram of the CRBRP. 
an attempt to model mixing in a pipe while the flow rate was increasing. The mixing option has been deleted.

The original model of the steam drum has been modified to include the possibility of condensation following separation of the two-phase evaporator outlet flow into saturated steam and water at the steam drum inlet. The saturated water mixes with subcooled recirculating water in the drum, not at a fictitious junction below the drum, so the water mixture in the drum is subcooled. The alternative to condensation during a pressure transient is therefore not evaporation but superheating. A number of test runs showed that condensation could be important but that superheating was negligible. Accordingly, the steam in the drum is assumed to remain saturated, and the model contains logic which uses all computed positive values of the condensation rate WC but which substitutes zero for any negative values of WC (Hetrick, Shinaishin, and Wait, 1978).

A major deficiency of the original model was the representation of the turbine-condenser-feedwater loop by a simple energy balance containing a constant plant efficiency. The most important consequence of this simplification is that any information, such as a pressure or temperature perturbation, is propagated too rapidly around the turbinecondenser-feedwater 1oop.

The other extreme, which would be reasonable during the early stages of a transient (especially for a transient initiated by an event in the primary loop), would be to treat the feedwater enthalpy HS5 as constant. An intermediate strategy would be to regard HS5 
as the turbine exit enthalpy delayed by some effective transport delay. As discussed in the next section, these three options are available in this version of BRENDA, and the user should be aware of the limitations of all three.

The original model neglected the thermal inertia of the fuel clad, assuming instead a quasistatic radial fuel-pin temperature distribution. The present version includes a differential equation for the average clad temperature TACL. Other quasistatic approximations remain, however. The reactor fuel is treated as a single lumped region, and the spatial peak temperatures are calculated from the space-average temperature TAF by assuming static temperature distributions.

Automatic controllers, modelled from simple frequency-domain descriptions together with appropriate dead bands and limiters, regulate the following:

1. Turbine control valve (TCV)

2. Steam dump bypass valve (VDMP)

3. Steam drum water level (YS5)

4. Intermediate and primary pumps

5. Reactor control assemblies

The latter (assemblies of neutron absorbers) respond to operator action or to automatic controllers that sense the following:

1. Turbine inlet steam temperature 
2. Reactor coolant outlet temperature

3. Reactor power

Independent safety systems that shut down the reactor, dump steam, and change pump speeds in response to abnormal signals from a variety of sensors are not included in this model.

The original set of controllers (Shinaishin, 1976) has been modified by altering gains and time constants and by replacing a number of fast-acting systems by instantaneous responses. The latter change, which greatly reduced the stiffness of the overall system, resulted in significant reduction of computer costs.

The original simulator also provided for stepwise motion of reactor control rods, which was derived from a continuous reactivity computation. Simulation of the stepping motion proved to be both costly and unnecessary, and it has been eliminated in this version of BRENDA.

The next two sections contain instructions for using BRENDA in conjunction with DARE P. This is followed by sample output for the response of the plant to a $10-c e n t$ reactivity step. Appendix A contains a dictionary of variables, Appendix $B$ is a 1isting of BRENDA, and Appendix C contains a discussion of computer graphics. 
HOW TO RUN PLANT TRANSIENTS

Provisions are made within the program for initializing common transients. These transients are:

Reactivity insertions

Opening the steam dump valve

Opening or closing the turbine control valve

Change in the electric load

The transients are initialized by specifying values in the initial conditions block of the DARE $P$ input file (BRENDA). These values are used in procedure blocks found at the beginning of the BRENDA 1isting in Appendix B.

In general, each procedure block controls one type of transient through the use of three control variables. The first variable begins with the letters EN (ENROC, ENDMP, ENV9, ENLD) and can assume the values 0,1 , or 2 where

$$
\begin{aligned}
& 0=\text { No transient } \\
& 1=\text { Step change } \\
& 2=\text { Ramp change }
\end{aligned}
$$

Thus, for a step in reactivity $E N R O C=1.0$. The second control variable begins with the letters DLT and represents the size of the change in the driven variable. DLTROC $=10.0$ is a 10 -cent change in reactivity for a step change, or a 10-cent/sec ramp rate for a ramp change. The third control variable begins with an $S$ and is used only for ramp changes $(\operatorname{ENXXX}=2,0)$. It is the duration of the ramp in seconds; 
SROC $=5.0$ would signify a ramp terminated at 5.0 seconds. A summary of the control variables follows.

\section{Contro1 Variable Summary}

Reactivity Transients

ENROC = transient type selector

DLTROC = reactivity change (cents or cents/sec)

SROC = total ramp duration (sec)

Dump Valve Transients

ENDMP = transient type selector

DLTDMP = change in normalized valve stroke $(\%$ or $\% / \mathrm{sec})$

SSVDMP $=$ total ramp duration

Turbine Control Valve Transients

ENV9 = transient type selector

DLTV9 = change in normalized valve stroke (\% or $\% / \mathrm{sec})$

SSV9 = total ramp duration

Electric Load Transients

ENLD = transient type selector

DLTLD = change in demand (\% or $\% / \mathrm{sec})$

SLD = total ramp duration

Examples:

$10 \%$ step decrease in electric demand:

$$
\text { ENLD }=1.0 \quad \text { DLTLD }=-10.0
$$

$3 \%$ ramp decrease in electric demand for ten seconds:

$$
\text { ENLD }=2.0 \quad \text { DLTLD }=3.0 \quad \text { SLD }=10.0
$$

10 cent step reactivity insertion (the transient which is produced by the listing as it appears in Appendix B):

$$
\text { ENROC }=1.0 \quad \text { DLTROC }=10.0
$$

Opening of one dump valve in four seconds:

$$
\text { ENDMP }=2.0 \quad \text { DLTDMP }=0.25 \quad \text { SSVDMP }=4.0
$$


Note that the source code for BRENDA as provided is initialized for the 10 cent step reactivity insertion. To run a different transient, delete ENROC $=1.0$ and insert the value 1.0 or 2.0 for the desired EN variable (plus values for DLT and S variables as appropriate). Note that the value of a parameter that is not specified will be taken to be zero.

One of the user input choices is the method used to calculate the feedwater enthalpy. The control variable used to specify the desired option is ENHS5. The default value of ENHS5 is zero and it may also assume the values one or two. The three choices are:

ENHS5 $=0.0$ (default)

Constant feedwater enthalpy.

ENHS $5=1.0$

Time-delayed propagation of the condenser output enthalpy, calculated by assuming a constant plant efficiency. The delay time TAUHS5 must be specified by the user.

ENHS5 $=2.0$

Instantaneous propagation of the condenser output enthalpy.

If ENROC $=1.0$ is deleted from the input file, the resulting output will be a steady state. A change in an initial value of a variable or in the values of certain parameters will upset this balance. Thus the response to a step change in any variable may be obtained by simply assigning a new initial value to that variable. However, new values for parameters may require new steady-state values for certain variables, compensating changes in other parameters, or both. 
ISOLATING PORTIONS OF THE PLANT

It is possible, with minor modification, to isolate sections or components of the CRBRP simulator. This is accomplished by breaking the logical connections between the components, and replacing the logical inputs to components by desired external inputs. As an example, consider a heat exchanger.

The logical connections in the model would conform to the physical connections of the physical system, i.e., the pipes. In BRENDA the pipes are modelled as pure transport delays, and the inlet temperature to a region will be defined with a statement of the form:

$$
\mathrm{T}_{\text {in }}=\operatorname{PDELAY}\left(\mathrm{T}_{\text {out }}, \mathrm{TAU}, \mathrm{m}, \mathrm{TI}_{\text {in }}\right)
$$

In this statement,

$T_{\text {in }}=$ the inlet temperature for the component.

$\mathrm{T}_{\text {out }}=$ the exit temperature from the component upstream.

$\mathrm{TAU}=$ the travel time for the fluid in the pipe.

m = an index number, different for each pipe.

$\mathrm{TI}_{\text {in }}=$ the initial inlet temperature, from fluid initially in the
pipe. Thus, to break this "connection" the above statement should be commented out and another statement inserted, of the form

$$
\mathrm{T}_{\text {in }}=\mathrm{T}_{\text {in }}(\mathrm{t}) \text {, }
$$

where $\mathrm{T}_{\text {in }}(t)$ is a user desired function (analytic or tabular).

The other logical "connection" between components is the flow rate from one component, through a pipe, and through the next component. 
Flow rates are defined in BRENDA for the various loops in the CRBRP, and the statements defining the flow through a component to be isolated should be located and replaced with user desired functions.

These two types of logical connections are the most common, but others also exist. For example, the reactor is coupled to the turbine control valve through the reactivity controller. Care should be taken to break these types of connections as required.

In an effort to assist the user in the identification of inputs to components, they are generally placed at the beginning of each component section in BRENDA. They are also identified with comment cards in the program.

To provide an example, consider the intermediate heat exchanger (IHX). Referring to Fig. 1, the two inlets to the IHX are the primary sodium inlet (P5) and the intermediate sodium inlet (II). The IHX section of BRENDA begins on line 5970. The primary inlet temperature is defined at line 6060 and the intermediate inlet temperature is defined at line 6020. The primary and intermediate flow rates are defined at lines 8960 and 9780 respectively.

The initial values for TP5 and TIl are listed in the initial conditions section, and initial values of WP and WI can be calculated from initial values of WPS and WIS using

$$
\begin{aligned}
& W P=3600.0 * \text { WPS } \\
& W I=3600.0 * \text { WIS. }
\end{aligned}
$$

These four variables define the inputs to the IHX. The user is free 
to remove 1 ines $6020,6060,8960$ and 9780 and replace them with other functions as desired. To study the effect of the primary flow rate on the secondary exit temperature, TP5, TI1 and WI would be set to their initial values for all time and WP would be set to a given function, such as a ramp in time.

The most convenient way to accomplish this change is through the use of procedure blocks. Procedure blocks are used to initialize transients, and examples may be found at the beginning of BRENDA, lines 220-570. The use of procedure blocks is explained in the DARE P USERS MANUAL, which, together with the DARE $P$ program, is available from Dr. John Wait, Department of Electrical Engineering, University of Arizona. 
SAMPLE OUTPUT

The sixteen pages of output listing that follow were generated by the sixteen LIST commands that appear on the last page of Appendix $B$. Line 14320 of Appendix B contains the statement $\operatorname{TMAX}=50.0$, and 1ine 14330 contains NPOINT $=51$, whence the output variables are printed for every second during the simulation.

The sample transient is the response of the CRBRP to a 10-cent reactivity step, with the option of constant feedwater enthalpy HS5. ENROC $=1.0$ and DLTROC $=10.0$ (1ine 15200 of Appendix $\mathrm{B}$ ), whereas ENHS5 has its default value of zero (see the procedure block, lines 51305170). In this version of BRENDA, reactivity steps or ramps are modelled as motion of the eccentric control rod (reactivity ROEXCD), while the reactivity controller operates through the main control assembly (position $\mathrm{X}$ and reactivity $\mathrm{RHOCD}$ ) .

Following the listings, selected output variables are plotted in Figs. 2-5, using a Tektronix Model 4010-1 graphics terminal and a copy maker. Each figure is a composite of two screen images. Figure 5 illustrates two of the many graphics options (four separate flow-rate graphs, and one graph with two sodium temperatures exhibiting the 34-sec delay time TAUTIA). The units used in Fig. 2-5 are: turbine power in megawatts, pressure in psia, temperatures in ${ }^{\circ} \mathrm{F}$, flow rates in $1 \mathrm{bm} / \mathrm{hr}$, heights in $\mathrm{ft}$, reactor power normalized to 975 megawatts (thermal), and reactivity in dollars. 
Figure 4 reflects one disadvantage of using automatic plotting in the presence of a discontinuity: the reactivity curve should descend smoothly from 0.1 and not show an apparent peak at $1 \mathrm{sec}$. Also, the true reactor power peaks at 1.11 well before $1 \mathrm{sec}$. This could be remedied by minor editing of the data files before plotting. 
TIME

1. $00000 \mathrm{~B}+00$

2. $00000 \mathrm{E}+00$

3. $00000 \mathrm{E}+00$

4. $00000 \mathrm{E}+00$

5. $00000 \mathrm{E}+00$

$6.00000 \mathrm{E}+00$

7. $00000 \mathrm{E}+00$

8. $00000 \mathrm{E}+00$

$9.00000 \mathrm{E}+00$

$1.00000 \mathrm{E}+01$

1. $10000 E+01$

$1.20000 \mathrm{E}+01$

1. $30000 \mathrm{E}+01$

1. $40000 \mathrm{E}+01$

1. $50000 \mathrm{E}+01$

$1.60000 \mathrm{E}+01$

1. $70000 \mathrm{E}+01$

$1.80000 \mathrm{E}+01$

$1.90000 \mathrm{E}+01$

2. $00000 \mathrm{E}+01$

2. $10000 \mathrm{E}+01$

$2.20000 E+01$

2. $30000 \mathrm{~B}+01$

$2.40000 E+01$

$2.50000 \mathrm{E}+01$

2. $60000 E+01$

$2.70000 \mathrm{E}+01$

2. $80000 \mathrm{E}+01$

$2.90000 \mathrm{E}+01$

3. $00000 \mathrm{E}+01$

3. $10000 \mathrm{E}+01$

3. 20000E+01

3. $30000 \mathrm{E}+01$

$3.40000 \mathrm{E}+01$

$3.50000 \mathrm{~B}+01$

$3.60000 \mathrm{E}+01$

$3.70000 \mathrm{E}+01$

$3.80000 \mathrm{E}+01$

$3.90000 \mathrm{E}+01$

4. $00000 \mathrm{E}+01$

4. $10000 E+01$

4. $20000 \mathrm{E}+01$

4. $30000 \mathrm{~B}+01$

$4.40000 \mathrm{E}+01$

$4.50000 \mathrm{E}+01$

$4.60000 \mathrm{E}+01$

4. $70000 E+01$

$4.80000 \mathrm{E}+01$

4. $90000 \mathrm{E}+01$

$5.00000 E+01$
PET AG

$2.55703 \mathrm{E}+01$

2. $55703 E+01$

2. $55702 \mathrm{E}+01$

2. $55700 \mathrm{E}+01$

$2.55722 \mathrm{E}+01$

2. $55816 \mathrm{~B}+01$

$2.55994 \mathrm{E}+01$

$2.56225 E+01$

2. $56463 E+01$

2. $56670 \mathrm{E}+01$

2. $56836 \mathrm{E}+01$

$2.56964 \mathrm{E}+01$

2. $57062 E+01$

$2.57134 \mathrm{E}+01$

$2.57182 E+01$

$2.57205 \mathrm{E}+01$

2. $57204 E+01$

$2.57184 \mathrm{E}+01$

$2.57150 E+01$

$2.57107 \mathrm{E}+01$

$2.57058 E+01$

$2.57005 E+01$

2. $56949 E+01$

$2.56889 \mathrm{E}+01$

2. $56827 \mathrm{E}+01$

2. $56763 E+01$

2. $56699 \mathrm{E}+01$

2. $56634 E+01$

$2.56570 \mathrm{E}+01$

2. $56506 \mathrm{E}+01$

2. $56443 \mathrm{E}+01$

$2.56380 \mathrm{E}+01$

$2.56317 \mathrm{E}+01$

$2.56255 E+01$

$2.56193 \mathrm{E}+01$

2. $56133 E+01$

2. $56075 E+01$

2. $56019 \mathrm{E}+01$

$2.55967 \mathrm{E}+01$

2. $55905 \mathrm{E}+01$

2. $55822 \mathrm{E}+01$

$2.55730 E+01$

2. $55648 \mathrm{E}+\mathrm{C} 1$

$2.55583 E+01$

. $55534 \mathrm{E}+01$

2. $55495 E+01$

2. $55460 \mathrm{E}+01$

2. $55424 \mathrm{E}+01$

2. $55385 \mathrm{E}+01$

2. $55342 E+01$

2. $552980+n 1$
OMGG

3. $76990 \mathrm{E}+02$

3. $76990 \mathrm{E}+02$

3. $76990 \mathrm{E}+02$

3. $76990 E+02$

3. $76990 \mathrm{E}+02$

3. $76990 \mathrm{E}+02$

3. $76990 \mathrm{E}+02$

3. $76990 \mathrm{E}+02$

3. $76990 \mathrm{E}+02$

$3.76990 \mathrm{E}+02$

3. $76990 \mathrm{E}+02$

$3.76990 \mathrm{E}+02$

3. $76990 \mathrm{E}+02$

$3.76990 \mathrm{E}+02$

3. $76990 \mathrm{E}+02$

$3.76990 E+02$

3. $76990 \mathrm{E}+02$

3. $76990 E+02$

3. $76990 \mathrm{E}+02$

3. $76990 \mathrm{E}+02$

3. $76990 \mathrm{E}+02$

3. $76990 \mathrm{E}+02$

3. $76990 \mathrm{E}+02$

3. $76990 \mathrm{E}+02$

3. $76990 \mathrm{E}+02$

3. $76990 E+02$

3. $76990 \mathrm{E}+02$

3. $76990 \mathrm{E}+02$

3. $76990 \mathrm{E}+02$

$3.76990 \mathrm{E}+02$

3. $76990 \mathrm{E}+02$

3. $76990 \mathrm{E}+02$

3. $76990 \mathrm{E}+02$

3. $76990 \mathrm{E}+02$

3. $76990 \mathrm{E}+02$

$3.76990 \mathrm{E}+02$

3. $76990 E+02$

$3.76990 \mathrm{E}+02$

3. $76990 \mathrm{E}+02$

$3.76990 \mathrm{E}+02$

3. $76990 \mathrm{E}+02$

3. $76990 \mathrm{E}+02$

3. $76990 \mathrm{E}+02$

3. $76990 \mathrm{E}+02$

3. $76990 \mathrm{E}+02$

$76990 \mathrm{E}+02$

3. $76990 \mathrm{E}+02$

3. $76990 E+02$

3. $76990 \mathrm{E}+02$

3. $76990 \mathrm{E}+02$

$3.76990 \mathrm{E}+02$
PGFET A

3. $80000 \mathrm{E}+02$

$3.80000 \mathrm{E}+02$

3. $79998 \mathrm{E}+02$

$3.79995 \mathrm{E}+02$

$3.80027 \mathrm{E}+02$

$80157 \mathrm{E}+02$

3. $80403 \mathrm{E}+02$

$3.80723 E+02$

$3.81052 E+02$

$3.81340 E+02$

$569 E+02$

$.81746 \mathrm{E}+02$

$3.81882 \mathrm{E}+02$

3. $81982 \mathrm{~B}+02$

$3.82049 E+02$

$3.82081 \mathrm{E}+02$

3. $82079 \mathrm{E}+02$

$3.82051 \mathrm{E}+02$

$3.82004 \mathrm{E}+02$

$3.81944 \mathrm{E}+02$

$3.81877 \mathrm{E}+02$

(2)

$3.81725 E+0$

$3.81557 \mathrm{E}+02$

$3.81469 \mathrm{E}+02$

$3.81379 \mathrm{R}+02$

$3.81290 \mathrm{E}+02$

$3.81200 \mathrm{E}+02$

$3.81112 \mathrm{E}+02$

$3.81024 \mathrm{E}+02$

$3.80938 E+02$

$3.80765 \mathrm{~B}+02$

$3.80679 \mathrm{E}+02$

$3.80596 \mathrm{E}+02$

$3.80515 \mathrm{E}+02$

$3.80438 \mathrm{E}+02$

$3.80365 E+02$

$3.80279 \mathrm{E}+02$

3. $80164 \mathrm{E}+02$

$3.80037 \mathrm{E}+02$

3. $79923 \mathrm{E}+02$

3. $79833 E+02$

$79711 E+02$

$3.79662 \mathrm{~B}+02$

3. $79613 \mathrm{~B}+02$

3. $79559 \mathrm{E}+02$

$3.79500 E+02$

$3.79438 \mathrm{E}+02$
$.60000 \mathrm{E}+00$

$7.60000 E+00$

$7.60000 \mathrm{E}+00$

$7.60000 \mathrm{E}+00$

$7.60000 \mathrm{~B}+00$

$7.60000 \mathrm{E}+00$

$7.60001 \mathrm{E}+00$

$7.60001 \mathrm{E}+00$

$7.60001 \mathrm{E}+00$

$7.60001 \mathrm{~B}+00$

$7.60000 \mathrm{E}+00$

$7.60000 \mathrm{E}+00$

$7.60000 \mathrm{E}+00$

$7.60000 \mathrm{~B}+00$

$7.60000 \mathrm{E}+00$

$7.60000 \mathrm{E}+00$

$7.60000 \mathrm{E}+00$

$7.60000 \mathrm{E}+00$

$7.60000 \mathrm{E}+00$

$7.60000 \mathrm{E}+00$

$7.60000 \mathrm{E}+00$

$7.60000 \mathrm{E}+00$

$760000=00$

$7.60000 \mathrm{E}+00$

$7.60000 \mathrm{E}+00$

$7.60000 \mathrm{E}+00$

$.60000 \mathrm{E}+00$

$60000 E+00$

$7.60000 \mathrm{E}+00$

$7.60000 \mathrm{E}+00$

$7.60000 \mathrm{E}+00$

$7.60000 \mathrm{E}+00$

$.60000 E+00$

$60000 \mathrm{E}+00$

$7.60000 \mathrm{E}+00$

$7.60000 \mathrm{E}+00$

$7.60000 \mathrm{E}+00$

$7.60000 \mathrm{E}+00$

7. $60000 \mathrm{E}+00$

$7.60000 \mathrm{E}+00$

$7.60000 \mathrm{E}+00$

$7.60000 \mathrm{E}+00$

$7.60000 \mathrm{E}+00$

$7.60000 \mathrm{E}+00$

$7.60000 \mathrm{E}+00$

$7.60000 \mathrm{E}+00$

$7.60000 \mathrm{E}+00$

$7.60000 \mathrm{E}+00$

$7.60000 \mathrm{E}+00$

$7.60000 \mathrm{E}+00$

$7.60000 \mathrm{E}+00$

0 .

$-2.99714 \mathrm{E}-05$

$-1.90792 \mathrm{E}-04$

$2.45692 \mathrm{E}-04$

$.94251 \mathrm{E}-03$

$98466 \mathrm{~B}-0$

$1.21949 \mathrm{E}-02$

. $39925 \mathrm{E}-02$

. $30829 \mathrm{E}-02$

$1.07986 \mathrm{E}-02$

$39749 \mathrm{E}-03$

$6.43958 \mathrm{E}-03$

$.88814 \mathrm{E}-03$

$3.50163 \mathrm{E}-03$

$2.05516 \mathrm{E}-03$

6. $10384 \mathrm{E}-04$

$-6.71326 \mathrm{E}-04$

$-1.63230 \mathrm{E}-03$

$-2.26917 \mathrm{E}-03$

$-2.66944 \mathrm{E}-03$

$.94207 \mathrm{E}-03$

$-3.16176 \mathrm{E}-03$

$-3.35410 \mathrm{E}-03$

$0.51500 \mathrm{~B}-03$

$-3.63434 \mathrm{E}-03$

$-3.70763 \mathrm{E}-0$

$3.73872 \mathrm{E}-03$ 
0. $2.00000 \mathrm{E}+00$ $3.00000 \mathrm{E}+00$ $4.00000 \mathrm{E}+00$ 5. $00000 \mathrm{E}+00$ $6.00000 \mathrm{E}+00$ 7. $00000 \mathrm{~B}+00$ $8.00000 \mathrm{~B}+00$ 9. $00000 \mathrm{E}+00$ $1.00000 E+01$ 1. $10000 \mathrm{E}+01$ 1. $20000 \mathrm{E}+01$ 1. $30000 \mathrm{E}+01$ $1.40000 \mathrm{E}+01$ $1.50000 \mathrm{E}+01$ $1.60000 \mathrm{E}+01$ $1.70000 \mathrm{E}+01$ $1.80000 \mathrm{E}+01$ 1. $90000 E+01$ $2.00000 \mathrm{E}+01$ 2. $10000 \mathrm{~B}+01$ $2.20000 \mathrm{E}+01$ $2.30000 \mathrm{E}+01$ $2.40000 \mathrm{E}+01$ $2.500008+01$ $2.60000 \mathrm{E}+01$ $2.70000 \mathrm{E}+01$ $2.80000 \mathrm{~B}+01$ 2. $90000 \mathrm{E}+01$ $3.00000 \mathrm{E}+01$ 3. $10000 E+01$ $3.20000 \mathrm{E}+01$ 3. $30000 \mathrm{E}+01$ $3.40000 \mathrm{E}+01$ $3.50000 \mathrm{E}+01$ $3.60000 \mathrm{E}+01$ $3.70000 \mathrm{~B}+01$ $3.80000 \mathrm{~B}+01$ $3.90000 \mathrm{E}+01$ 4. $00000 \mathrm{E}+01$ 4. 10000E+01 $4.20000 \mathrm{~B}+01$ 4. $30000 E+01$ $4.40000 \mathrm{~B}+01$ 4. $50000 \mathrm{E}+01$ $4.60000 \mathrm{E}+01$ 4. $70000 E+01$ 4. $80000 \mathrm{E}+01$ 4. $90000 Z+01$ 5. $00000 \mathrm{E}+01$
159 3. $34000 E+06$ 3. $34000 E+06$ $3.33996 \mathrm{E}+06$ 3. 34000E+06 3. $34049 \mathrm{E}+06$ 3. $34171 \mathrm{E}+06$ 3. $34365 \mathrm{E}+06$ 3. $34603 E+06$ 3. $34842 \mathrm{~B}+06$ 3. $35059 \mathrm{E}+06$ 3. $35246 \mathrm{E}+06$ 3. $35408 \mathrm{E}+06$ 3. $35545 \mathrm{E}+06$ 3. $35657 \mathrm{E}+06$ 3. $35741 \mathrm{E}+06$ 3. $35796 \mathrm{E}+06$ 3. $35823 \mathrm{E}+06$ 3. $35827 \mathrm{E}+06$ $3.35812 \mathrm{E}+06$ 3. $35783 E+06$ 3. $35743 \mathrm{E}+06$ 3. $35692 \mathrm{E}+06$ 3. $35634 \mathrm{E}+06$ 3. $35568 \mathrm{~B}+06$ 3. $35498 \mathrm{E}+06$ 3. $35424 \mathrm{E}+06$ $3.35347 \mathrm{E}+06$ 3. $35268 \mathrm{E}+06$ 3. $35189 E+06$ 3. $35110 \mathrm{E}+06$ 3. $35030 \mathrm{E}+06$ 3. $34950 E+06$ 3. $34870 \mathrm{E}+06$ 3. $34790 \mathrm{E}+06$ 3. $34710 \mathrm{~B}+06$ 3. $34633 \mathrm{~B}+06$ 3. $34557 \mathrm{E}+06$ 3. $34486 E+06$ 3. $34418 \mathrm{E}+06$ 3. $34356 \mathrm{E}+06$ 3. $34301 \mathrm{E}+06$ 3. $34254 \mathrm{E}+06$ 3. $34211 \mathrm{E}+06$ 3. $34170 B+06$ 3. $34131 \mathrm{E}+06$ 3. $34093 \mathrm{E}+06$ $3.34051 \mathrm{E}+06$ $3.34002 \mathrm{E}+06$ 3. $33945 \mathrm{E}+06$ 3. $33881 E+06$ 3. $33812 \mathrm{E}+06$
PS 9

$.46470 E+03$

1. $46470 E+03$

1. $46468 B+03$

$1.46470 E+03$

1. $46494 \mathrm{E}+03$

$.465598+03$

$1.466678+03$

$1.468058+03$

1. $46948 \mathrm{E}+03$

$1.47082 \mathrm{E}+03$

1. $47200 \mathrm{E}+03$

$1.47302 \mathrm{E}+03$

$1.47391 \mathrm{E}+03$

$1.47466 \mathrm{~B}+03$

1. $47526 \mathrm{~B}+03$

$1.47569 \mathrm{E}+03$

1. $47597 \mathrm{E}+03$

$1.47611 \mathrm{E}+03$

1. $47615 E+03$

$1.47610 E+03$

1. $47598 \mathrm{~B}+03$

$1.47581 \mathrm{E}+03$

1. $47559 \mathrm{E}+03$

$1.47532 \mathrm{E}+03$

$1.47503 \mathrm{E}+03$

$1.47470 \mathrm{E}+03$

1. $47436 \mathrm{E}+03$

$1.47400 E+03$

1. $47364 \mathrm{~B}+03$

$1.47326 \mathrm{E}+03$

$1.47288 E+03$

$1.472498+03$

1. $47210 \mathrm{E}+03$

$1.47170 \mathrm{E}+03$

1. $47130 \mathrm{E}+03$

$1.47090 \mathrm{E}+03$

$1.47051 \mathrm{E}+03$

$1.47013 E+03$

1. $46977 \mathrm{E}+03$

1. $46943 \mathrm{~B}+03$

$1.46910 E+03$

$1.46878 \mathrm{~B}+03$

1. $46847 \mathrm{E}+03$

$1.46818 E+03$

1. $46792 \mathrm{E}+03$

$1.46766 E+03$

1. $46739 \mathrm{E}+03$

$1.46708 E+03$

1. $46673 \mathrm{E}+03$

$1.46634 \mathrm{E}+03$

$1.46593 \mathrm{E}+03$
TS9

$.00000 \mathrm{E}+02$

$.00000 \mathrm{E}+02$

. $00000 \mathrm{~B}+02$

9. $00000 \mathrm{E}+02$

$9.00037 \mathrm{~B}+02$

$9.00224 \mathrm{E}+02$

$.00224 E+02$

$9.00679 \mathrm{E}+02$

$9.00772 \mathrm{E}+02$

$9.00746 \mathrm{E}+02$

$9.00624 \mathrm{E}+02$

$9.00443 \mathrm{~B}+02$

$9.00235 E+02$

$9.00019 \mathrm{E}+02$

E. $99804 \mathrm{~B}+02$

B. $99592 \mathrm{E}+02$

8. $99391 E+02$

$8.99211 E+02$

$8.99061 E+02$

$8.98944 E+02$

8. 98856E+02

8. $98794 \mathrm{E}+02$

8. $98754 \mathrm{E}+02$

8. $98729 \mathrm{E}+02$

8. $98719 \mathrm{E}+02$

8. $98720 E+02$

8. $98730 \mathrm{E}+02$

8. $98749 \mathrm{E}+02$

8. $98774 \mathrm{E}+02$

E. $98805 E+02$

$\varepsilon-98840 \mathrm{E}+02$

8. $98878 E+02$

8. $98918 E+02$

8. $98961 E+02$

8. $99005 E+02$

$8.99050 \mathrm{E}+02$

$8.99097 \mathrm{E}+02$

8. $99146 E+02$

8. $99179 \mathrm{E}+02$

8. $99082 E+02$

E. $98844 E+02$

$8.98585 E+02$

6. $98392 \mathrm{E}+02$

8. $98266 \mathrm{E}+02$

8. $98243 \mathrm{E}+02$

8.98236E+02

$8.98251 \mathrm{E}+02$

8. $98283 E+02$

$8.98331 \mathrm{E}+02$

8. $98397 E+02$

8. $98483 \mathrm{E}+02$
Hs9

$43058 \mathrm{E}+03$

1. $43058 E+03$

1. $43058 E+03$

$1.43058 \mathrm{E}+03$

$1.430598+03$

$1.43068 \mathrm{E}+03$

$1.43079 \mathrm{~B}+03$

$1.43086 \mathrm{~B}+03$

$1.43086 \mathrm{E}+03$

1. $43079 \mathrm{E}+03$

$1.43067 E+03$

$1.43052 \mathrm{E}+03$

1.430

$1.43036 \mathrm{E}+03$

1. $43020 \mathrm{E}+03$

1. $43004 \mathrm{E}+03$

1. $42988 \mathrm{E}+03$

$1.429748+03$

1. $42962 \mathrm{E}+03$

1. $42952 E+03$

$1.42944 \mathrm{E}+03$

$1.42939 \mathrm{E}+03$

1. $42936 \mathrm{E}+03$

1. $42934 \mathrm{E}+03$

1. $42934 \mathrm{E}+03$

$1.42934 \mathrm{E}+03$

1. $42935 \mathrm{E}+03$

$1.42937 \mathrm{E}+03$

1. $42940 \mathrm{E}+03$

$1.42943 \mathrm{E}+03$

$1.42947 E+03$

$1.42950 \mathrm{E}+03$

1. $42954 \mathrm{E}+03$

$1.42958 E+03$

$1.42963 E+03$

$1.42967 \mathrm{E}+03$

$1.42972 \mathrm{E}+03$

$1.42976 \mathrm{E}+03$

$1.42981 \mathrm{E}+03$

$1.42984 \mathrm{E}+03$

1. $42980 \mathrm{E}+03$

$1.42965 E+03$

1. $42950 \mathrm{E}+03$

$1.42938 E+03$

1. $42933 E+03$

$1.42931 \mathrm{E}+03$

1. $42932 \mathrm{E}+03$

1. $42934 E+03$

$1.42937 E+03$

$1.42941 \mathrm{E}+03$

1. $42947 \mathrm{E}+03$

1. $42954 E+03$ 
1. $00000 \mathrm{E}+0$

$2.00000 \mathrm{E}+00$

3. $00000 E+00$

4. $00000 \mathrm{E}+00$

$5.00000 E+00$

$6.00000 \mathrm{E}+00$

$7.00000 \mathrm{E}+00$

$8.00000 \mathrm{E}+00$

$9.00000 \mathrm{E}+00$

$1.00000 E+01$

1. $10000 \mathrm{E}+01$

1. $20000 \mathrm{E}+01$

$1.30000 E+01$

$1.40000 \mathrm{E}+01$

$1.50000 \mathrm{E}+01$

$1.60000 \mathrm{~B}+01$

$1.70000 E+01$

$1.80000 E+01$

$1.90000 \mathrm{~B}+01$

$2.00000 \mathrm{~B}+01$

$2.10000 \mathrm{E}+01$

$2.20000 \mathrm{E}+01$

$2.30000 E+01$

$2.40000 E+01$

$2.50000 E+01$

$2.60000 \mathrm{E}+01$

$2.70000 \mathrm{~B}+01$

$2.80000 E+01$

$2.90000 E+01$

$3.00000 E+01$

3. $10000 \mathrm{E}+01$

3. $20000 \mathrm{E}+01$

$3.30000 E+01$

$3.40000 \mathrm{E}+01$

$3.50000 E+01$

$3.60000 E+01$

3. $70000 E+01$

$3.80000 \mathrm{E}+01$

$3.90000 \mathrm{E}+01$

4. $00000 \mathrm{E}+01$

4. $10000 E+01$

$4.20000 E+01$

4. $30000 E+01$

$4.40000 E+01$

$4.50000 E+01$

$4.60000 E+01$

$4.70000 E+01$

$4.80000 E+01$

$4.90000 \mathrm{E}+01$

$5.00000 E+01$
USC

$34000 E+06$

3. $34000 E+06$

3. $33996 \mathrm{E}+06$

3. $34000 \mathrm{E}+06$

$34049 E+06$

3. $34171 \mathrm{E}+06$

$34365 E+06$

3. $34603 \mathrm{E}+06$

3. $34842 E+06$

3. $35059 E+06$

. $35246 \mathrm{E}+06$

3. $35408 \mathrm{E}+06$

3. $35545 E+06$

$35657 \mathrm{E}+06$

3. $35741 \mathrm{E}+06$

$3.35796 \mathrm{E}+06$

3. $35823 E+06$

3. $35827 \mathrm{E}+06$

$35812 \mathrm{E}+06$

3. $35783 \mathrm{E}+06$

3. $35743 \mathrm{E}+06$

3. $35692 \mathrm{E}+06$

3. $35634 \mathrm{E}+06$

3. $35568 \mathrm{E}+06$

$35498 E+06$

3. $35424 \mathrm{E}+06$

3. $35347 \mathrm{E}+06$

3. $35268 E+06$

3. $35189 \mathrm{E}+06$

3. $35110 \mathrm{E}+06$

$35030 E+06$

3. $34950 \mathrm{E}+06$

3. $34870 \mathrm{E}+06$

3. $34790 \mathrm{E}+06$

3. $34710 \mathrm{E}+06$

3. $34633 E+06$

3. $34557 E+06$

$34486 \mathrm{E}+06$

3. $34418 \mathrm{E}+06$

3. $34356 \mathrm{E}+06$

3. $34301 \mathrm{E}+06$

3. $34254 \mathrm{E}+06$

$34211 E+06$

$34170 \mathrm{E}+06$

. $341318+06$

3.3409

3. $34051 E+06$

3. $34002 \mathrm{E}+06$

3. $33945 E+06$

. $33881 \mathrm{E}+06$

$3.33912 E+06$
TASA

TAHA

$7.601248+02$

$7.60217 \mathrm{E}+02$

$7.60345 \mathrm{E}+02$

7. $60460 \mathrm{E}+02$

$7.60540 \mathrm{E}+02$

$7.60582 \mathrm{E}+02$

$.60590 \mathrm{E}+02$

$7.60571 \mathrm{E}+02$

7. $60533 \mathrm{E}+02$

7. $60483 \mathrm{E}+02$

$60426 \mathrm{E}+02$

7. $60363 E+0$

$7.60299 E+02$

7. $60238 E+02$

$7.60184 \mathrm{E}+02$

$7.60138 E+02$

7. $60100 \mathrm{E}+02$

7. $60070 E+02$

$7.60045 \mathrm{E}+02$

$7.60026 E+02$

7. $60011 \mathrm{E}+02$

7. $59999 \mathrm{E}+02$

$7.59989 \mathrm{E}+02$

7. $59983 \mathrm{E}+02$

$7.59978 \mathrm{E}+02$

7. $59975 E+02$

$7.59973 \mathrm{E}+02$

7. $59972 \mathrm{E}+02$

$7.59973 \mathrm{E}+02$

$7.59974 E+02$

7. $59975 \mathrm{E}+02$

7. $59977 \mathrm{E}+02$

$7.59980 \mathrm{E}+02$

$7.59983 \mathrm{E}+02$

$7.59978 \mathrm{E}+02$

$7.59907 E+02$

$7.59767 \mathrm{E}+02$

7. $59618 \mathrm{E}+02$

7. $59505 E+02$

7. $59436 \mathrm{E}+02$

$7.59399 \mathrm{E}+02$

$7.59381 \mathrm{E}+02$

$7.59375 \mathrm{E}+02$

7. $59378 \mathrm{E}+02$

$7.59389 \mathrm{E}+02$

7. $59409 \mathrm{E}+02$

7. $59437 \mathrm{E}+02$

$7.59470 \mathrm{E}+02$

7. $59501 \mathrm{E}+02$

$7.59530 \mathrm{E}+02$
$5 \dot{0}+02$

$.72300 \mathrm{E}+02$

8. $72456 \mathrm{E}+02$

8.72645E+02

8. $72804 E+02$

$8.72908 \mathrm{E}+02$

. 72964E+02

$72986 \mathrm{E}+02$

8. $72983 \mathrm{E}+02$

$8.72961 E+02$

$8.72923 E+02$

$8.72870 \mathrm{E}+02$

8.72806E+02

$8.72736 \mathrm{E}+02$

8. $72669 \mathrm{E}+02$

8. $72608 \mathrm{E}+02$

8. $72555 \mathrm{E}+02$

8.72510E+02

8. $72470 E+02$

$8.72436 E+02$

8.72406B+02

8. $72379 \mathrm{E}+02$

$8.72355 \mathrm{E}+02$

$8.72333 \mathrm{E}+02$

$8.72314 \mathrm{E}+02$

$8.72296 \mathrm{E}+02$

$8.72281 \mathrm{E}+02$

$8.72266 E+02$

$8.72253 \mathrm{E}+02$

$8.72241 \mathrm{E}+02$

8. $72230 \mathrm{E}+02$

$8.72220 \mathrm{E}+02$

$8.72211 E+02$

$8.72202 E+02$

$8.72195 E+02$

$8.72168 \mathrm{E}+02$

8. $72026 E+02$

$8.71794 E+02$

$8.71565 \mathrm{E}+02$

$8.71396 E+02$

$8.71294 \mathrm{E}+02$

$8.71237 \mathrm{E}+02$

8. 71207E+02

$8.71193 \mathrm{E}+02$

$8.71193 \mathrm{E}+02$

8.71206E+02

$8.71232 E+02$

$8.71271 \mathrm{E}+02$

8. $71313 \mathrm{E}+02$

$8.71353 \mathrm{E}+02$

$8.71366 \mathrm{E}+02$
TAIA

$95500 E+02$

8. $95544 E+02$

8. $95692 \mathrm{E}+02$

8. $95867 \mathrm{E}+02$

$8.96011 E+02$

8. $96105 E+02$

$8.96156 \mathrm{E}+02$

$8.96179 \mathrm{E}+02$

8. $96182 E+02$

8. $96168 \mathrm{E}+02$

$8.96139 E+02$

8. $96097 E+02$

$8.95984 E+02$

$8.95926 \mathrm{E}+02$

8. $95874 \mathrm{E}+02$

$8.95828 E+02$

8. $95788 \mathrm{E}+02$

8. $95753 E+02$

8. $95722 E+02$

8. $95693 \mathrm{E}+02$

$8.95668 E+02$

$8.95644 \mathrm{E}+02$

8. $95623 \mathrm{E}+02$

$8.95603 E+02$

$8.95585 E+02$

8. $95568 \mathrm{E}+02$

8. $95553 E+02$

8. $95538 E+02$

$8.95525 \mathrm{E}+02$

$8.95512 E+02$

8. $955008+02$

(2)

$8.95478 \mathrm{~B}+02$

$8.95469 E+02$

8. $95395 \mathrm{E}+02$

$8.95146 \mathrm{E}+02$

8. $94864 \mathrm{E}+02$

$8.94651 E+02$

8. $94516 E+02$

$8.94447 E+02$

$8.94410 \mathrm{E}+02$

$8.94391 \mathrm{E}+02$

8. $94385 E+02$

$8.94393 E+02$

$8.944168+02$

8. $94453 \mathrm{E}+02$

8. $94499 E+02$

$8.94543 E+02$

$94500 \mathrm{E}+02$

$8.94608 E+02$ 


\section{TIME}

$1.00000 \mathrm{E}+00$

2. $00000 E+00$

$3.00000 \mathrm{E}+00$

4. $00000 \mathrm{E}+00$

$5.00000 \mathrm{E}+00$

$6.00000 \mathrm{E}+00$

7. $00000 \mathrm{E}+00$

$8.00000 E+00$

$9.00000 \mathrm{E}+00$

1. $00000 \mathrm{E}+01$

1. $10000 \mathrm{E}+0$

$1.20000 E+01$

1. $30000 \mathrm{E}+01$

$1.40000 \mathrm{E}+01$

1. $50000 \mathrm{E}+01$

1. $60000 E+01$

$1.70000 \mathrm{E}+01$

$1.80000 \mathrm{E}+01$

1. $90000 \mathrm{E}+01$

$2.00000 E+01$

2. $10000 \mathrm{E}+01$

2. $20000 \mathrm{E}+01$

$2.30000 E+01$

$2.40000 E+01$

2. $50000 \mathrm{E}+01$

$2.60000 \mathrm{E}+01$

$2.70000 \mathrm{E}+01$

$2.80000 \mathrm{E}+01$

$2.90000 \mathrm{E}+01$

3. $00000 E+01$

3. $10000 \mathrm{E}+01$

3. $20000 \mathrm{E}+01$

3. $30000 \mathrm{E}+01$

$3.40000 \mathrm{E}+01$

$3.50000 E+01$

$3.60000 E+01$

3. $70000 \mathrm{E}+01$

3. $B 0000 E+01$

3. $90000 \mathrm{E}+01$

4. $00000 \mathrm{E}+01$

$4.10000 \mathrm{E}+01$

4. $20000 \mathrm{E}+01$

4. $30000 \mathrm{E}+01$

$4.40000 \mathrm{E}+01$

4. $50000 \mathrm{E}+01$

4. $60000 \mathrm{E}+01$

4. $70000 \mathrm{E}+01$

$4.80000 \mathrm{E}+01$

$4.90000 \mathrm{E}+0$

$5.00000 E+01$
PSA

$1.73970 \mathrm{E}+03$

$1.73970 \mathrm{E}+03$

$1.73967 \mathrm{E}+03$

1. $73970 \mathrm{E}+03$

1. $714003 \mathrm{E}+03$

$1.74088 \mathrm{E}+03$

1. $74228 \mathrm{E}+03$

$1.74406 \mathrm{E}+03$

$1.74589 \mathrm{E}+03$

1. $74757 \mathrm{E}+03$

$1.74906 \mathrm{E}+03$

$1.75035 E+03$

$1.75147 \mathrm{E}+03$

$1.75240 \mathrm{E}+03$

$1.75314 \mathrm{E}+03$

$1.75366 \mathrm{E}+03$

1. $75398 \mathrm{E}+03$

$1.75413 E+03$

1. $75414 \mathrm{E}+03$

$1.75404 \mathrm{E}+03$

$1.75386 \mathrm{E}+03$

$1.75360 \mathrm{E}+03$

1. $75328 \mathrm{E}+03$

1. $75291 \mathrm{E}+03$

$1.75249 \mathrm{E}+03$

$1.75205 P+03$

1. $75158 \mathrm{E}+03$

$1.75109 \mathrm{E}+0.3$

$1.75059 \mathrm{E}+03$

$1.75009 \mathrm{E}+03$

$1.74957 \mathrm{E}+03$

$1.74906 \mathrm{E}+03$

$1.74853 \mathrm{~B}+03$

$1.748008+03$

$1.74746 \mathrm{E}+03$

1. $74694 E+03$

$1.74642 \mathrm{E}+03$

1. $74593 E+03$

$1.74546 \mathrm{E}+03$

$1.74501 \mathrm{E}+03$

$1.74459 E+03$

$1.74419 \mathrm{E}+03$

1. $74382 \mathrm{E}+03$

1. $74346 \mathrm{E}+03$

$1.74313 E+03$

$1.74281 \mathrm{E}+03$

$1.74247 \mathrm{E}+03$

$1.74208 \mathrm{E}+03$

$1.74164 \mathrm{E}+0.3$

$1.74114 \mathrm{E}+03$

1. $74061 \mathrm{E}+03$
PSC

$1.53970 \mathrm{E}+03$

$1.53970 \mathrm{E}+03$

1. $53968 \mathrm{E}+03$

$1.53970 \mathrm{E}+03$

1. $53997 \mathrm{E}+03$

$1.54067 E+03$

1. $54184 E+03$

$1.54333 E+03$

1. $54487 \mathrm{E}+03$

$1.54630 \mathrm{~B}+03$

1. $54756 \mathrm{E}+03$

1. $54866 \mathrm{~B}+03$

$1.54961 \mathrm{E}+03$

1. $55041 \mathrm{E}+03$

1. $55104 \mathrm{~B}+03$

$1.55150 \mathrm{E}+03$

1. $55179 \mathrm{E}+03$

$1.55194 E+03$

1. $55196 E+03$

1. $55190 \mathrm{E}+03$

$1.55177 \mathrm{E}+03$

$1.55157 E+03$

1. $55132 \mathrm{E}+03$

$1.55103 E+03$

$1.55070 E+03$

1. $55034 \mathrm{E}+03$

$1.54996 \mathrm{t}+03$

1. $54957 \mathrm{E}+03$

1. $54917 E+03$

1. $54876 \mathrm{E}+03$

1. $54834 E+03$

$1.54792 \mathrm{E}+03$

$1.54749 \mathrm{E}+03$

1. $54705 \mathrm{E}+03$

1. $54661 \mathrm{E}+03$

1. $54618 \mathrm{E}+03$

$1.54576 E+03$

1. $54496 \mathrm{E}+03$

1. $54459 \mathrm{E}+03$

1. $54423 E+03$

1. $54389 E+03$

$1.54356 \mathrm{E}+03$

1. $54326 F+03$

1. $54297 \mathrm{E}+03$

1. $54270 \mathrm{E}+03$

1. $542418+03$

1. $54208 E+03$

$1.541715+03$

1. $54129 E+03$

1. $54084 \mathrm{~F}+03$
TSA

$6.15209 \mathrm{E}+02$ $6.15209 E+02$ $6.15207 \mathrm{~B}+02$ $6.15209 E+02$ 6. 15235E+02 $6.15303 E+02$ 6. $15414 \mathrm{E}+02$ $6.15554 \mathrm{E}+02$ 6.15699 $6.15833 \mathrm{E}+02$ 6. $15951 \mathrm{E}+02$ $6.16053 E+02$ 6. $16141 \mathrm{E}+02$ 6. $16215 \mathrm{E}+02$ $6.16273 E+02$ $6.16315 \mathrm{E}+02$ $6.16340 \mathrm{E}+02$ $6.16352 E+02$ 6. $16353 \mathrm{E}+02$ $6.16345 E+02$ $6.16330 \mathrm{E}+02$ $6.16310 \mathrm{E}+02$ 6. 16285E+02 6. 16255E+02 $6.16223 \mathrm{E}+02$ $6.16187 \mathrm{E}+02$ 6. $16150 \mathrm{E}+02$ $6.16111 E+02$ 6. $16072 \mathrm{E}+02$ 6. 16032E+02 $6.15991 \mathrm{E}+02$ 6. $15950 \mathrm{E}+02$ $6.15908 \mathrm{E}+02$ 6. $15866 \mathrm{E}+02$ 6. $15824 \mathrm{E}+02$ 6. $15782 \mathrm{E}+02$ $6.15742 E+02$ $6.15703 E+02$ 6. $15665 E+02$ $6.15630 \mathrm{E}+02$ 6. 15597E+02 $6.15565 E+02$ $6.15535 E+02$ $6.15507 \mathrm{E}+02$ 6. $15481 \mathrm{E}+02$ $6.15455 \mathrm{E}+02$ $6.15429 E+02$ 6. 15398E+02 6. 15363E+02 $6.15324 \mathrm{E}+02$ 6. $15282 \mathrm{E}+02$
T SC

$9.05000 E+02$ .05038E+0 $9.05227 \mathrm{E}+0$ $9.05480 \mathrm{E}+02$ $9.05684 \mathrm{E}+02$ $9.05777 \mathrm{E}+02$ $9.05749 E+02$ $9.05626 \mathrm{E}+02$ $9.05443 \mathrm{E}+02$ $9.05232 \mathrm{E}+02$ $9.05015 E+02$ $9.04798 E+02$ $9.04586 \mathrm{E}+02$ $9.04383 E+02$ $9.04203 E+02$ $9.04053 E+02$ $9.03935 \mathrm{E}+02$ $9.03848 \mathrm{E}+02$ $9.03786 \mathrm{E}+02$ $9.03746 \mathrm{E}+02$ $9.03722 \mathrm{E}+02$ $9.03712 \mathrm{E}+02$ $9.03713 E+02$ $9.03724 E+02$ $9.03743 E+02$ $9.03768 E+02$ $9.03799 \mathrm{E}+02$ $9.03834 \mathrm{E}+02$ $9.03873 E+02$ $9.03914 E+02$ $9.03956 \mathrm{E}+02$ $9.04000 \mathrm{E}+02$ $9.04046 \mathrm{E}+02$ $9.04093 \mathrm{E}+02$ $9.04143 E+02$ $9.04174 E+02$ $9.04073 E+02$ $9.03831 \mathrm{E}+02$ $9.03572 \mathrm{E}+02$ $9.03380 \mathrm{E}+02$ $9.03275 E+02$ $9.03233 \mathrm{E}+02$ $9.03227 \mathrm{E}+02$ $9.03242 \mathrm{E}+0$ $9.03274 \mathrm{E}+02$ $9.03323 \mathrm{E}+02$ $9.03390 \mathrm{E}+02$ $9.03477 \mathrm{E}+02$ $9.03577 \mathrm{E}+02$ $9.03679 \mathrm{E}+02$ $9.03777 \mathrm{E}+02$

HSC

$1.43071 \mathrm{E}+03$ $1.43074 \mathrm{E}+03$ 1.43085 $1.43101 E+03$ $1.43112 \mathrm{E}+03$ $1.43115 E+03$ $1.43109 E+03$ $1.43096 \mathrm{E}+03$ $1.43078 \mathrm{E}+03$ $1.43060 \mathrm{E}+03$ $1.43042 E+03$ $1.43024 E+03$ $1.43008 \mathrm{E}+03$ $1.42992 E+03$ 1. $42979 \mathrm{E}+03$ $142968 \mathrm{E}+03$ $1.42959 \mathrm{E}+03$ 1. $42953 \mathrm{E}+03$

$1.42950 E+03$

$1.42947 E+03$ $1.42946 \mathrm{E}+03$

$1.42946 \mathrm{E}+\mathrm{U3}$

$1.42947 E+03$ $1.42949 \mathrm{E}+03$ 
$1.00000 E+00$ $2.00000 \mathrm{~B}+00$ $00 E+00$ $3.00000 E+00$ a. $00000 \mathrm{E}+00$ $5.00000 \mathrm{E}+00$ $6.00000 E+00$ 7. $00000 E+00$ $8.00000 \mathrm{E}+00$ $9.00000 \mathrm{E}+00$ $1.00000 E+01$ $1.10000 \mathrm{E}+01$ $1.20000 E+01$ $1.30000 \mathrm{E}+01$ $1.40000 \mathrm{E}+01$ 1. $50000 \mathrm{E}+01$ $1.60000 \mathrm{E}+01$ $1.70000 E+01$ $1.80000 \mathrm{E}+01$ 1. $90000 E+01$ $2.000008+01$ 2. $10000 \mathrm{E}+01$ 2.20000E+01 $2.30000 E+01$ $2.40000 \mathrm{E}+01$ $2.50000 E+01$ $2.60000 E+01$ 2.70000E+01 $2.80000 \mathrm{E}+01$ $2.90000 E+01$ $3.00000 \mathrm{E}+01$ 3. 10000E+01 $3.20000 E+01$ 3. $30000 \mathrm{E}+01$ $3.40000 \mathrm{E}+01$ $3.50000 \mathrm{E}+01$ $3.60000 \mathrm{E}+01$ 3. $70000 \mathrm{E}+01$ $3.80000 \mathrm{E}+01$ 3. $90000 E+01$ 4. $00000 \mathrm{E}+01$ 4. $10000 E+01$ $4.20000 E+01$ 4. $30000 \mathrm{E}+01$ 4. $40000 E+01$ 4. $50000 \mathrm{E}+01$ 4. $60000 \mathrm{E}+01$ $4.70000 E+01$ $4.80000 \mathrm{E}+01$ 4. $90000 E+01$ $5.00000 E+01$
ELS 1 2. $13016 \mathrm{E}+0$ 2. $12985 E+01$ 2.12710E+0 $2.12155 \mathrm{E}+01$ . $11565 \mathrm{E}+0$ 2. $11182 E+0$ 2. $11099 \mathrm{E}+01$ 2. $11268 \mathrm{E}+01$ 2. $11565 E+0$ $2.118908+0$ $12202 \mathrm{E}+0$ 2. $12497 \mathrm{E}+0$ 2. $12784 E+0$ 2. $13061 \mathrm{~B}+01$ 2. $13311 \mathrm{E}+0$ 2. $13514 \mathrm{E}+0$ 2. $13661 \mathrm{E}+01$ $2.13759 \mathrm{E}+0$ 2. $13819 \mathrm{E}+01$ 2. $13856 \mathrm{~B}+01$ 2. $13879 \mathrm{E}+01$ $2.13894 \mathrm{~B}+01$ 2. $13902 \mathrm{E}+0$ $2.13907 \mathrm{E}+0$ 2. $13908 \mathrm{E}+0$ 2. 13907E+01 2. $13905 E+0$ 2. $13906 \mathrm{~B}+01$ $13916 \mathrm{E}+01$ $.13934 \mathrm{E}+0$ 2. $13957 \mathrm{E}+0$ 2. $13978 \mathrm{E}+01$ 2. $13990 \mathrm{E}+01$ 2. $13991 \mathrm{E}+01$ 2. $13981 \mathrm{E}+01$ . $13960 \mathrm{E}+0$ 2. $13931 \mathrm{~B}+01$ 2. $13896 \mathrm{~B}+0$ 2. $13859 \mathrm{E}+01$ 2. $13820 \mathrm{E}+0$ 2.13780E + 01 . $13739 E+01$ 2. $13699 \mathrm{z}+0$ 2. $13659 \mathrm{E}+01$ 2. $13624 \mathrm{E}+0$ 2. $13594 \mathrm{E}+0$ 2. $13564 E+0$ 2. $13529 \mathrm{E}+01$ 2. $13483 \mathrm{E}+0$ 2. $13428 \mathrm{E}+01$ 2. $13365 E+01$
ELS2

2. $46984 \mathrm{E}+01$

$015 E+0$

. $47290 \mathrm{E}+0$

$.47845 E+01$

$2.48435 E+01$

$2.48818 \mathrm{E}+0$

2. $48901 \mathrm{E}+01$

$2.48732 E+01$

$2.48435 E+0$

$2.48110 \mathrm{E}+01$

$2.47798 \mathrm{E}+01$

$2.47503 \mathrm{E}+01$

2. $47216 E+01$

$2.46939 \mathrm{E}+01$

2. $46689 E+01$

$2.46486 \mathrm{~B}+01$

2. $46339 \mathrm{E}+0$

$2.46241 \mathrm{E}+01$

$2.46181 \mathrm{E}+0$

$2.46144 E+01$

$2.46121 E+0$

2. $46106 \mathrm{~B}+01$

2. $46098 \mathrm{E}+0$

$2.46093 \mathrm{E}+01$

$2.46092 E+01$

$2.46093 \mathrm{E}+0$

$2.46095 E+0$

$2.46094 \mathrm{E}+01$

$2.46084 \mathrm{E}+0$

$2.46066 \mathrm{~B}+01$

$2.46043 E+01$

$2.46022 \mathrm{E}+01$

$2.46010 \mathrm{E}+0$

$2.46009 E+01$

$2.46019 \mathrm{E}+01$

$2.46040 \mathrm{E}+01$

2. $46069 E+01$

$2.46104 E+01$

$2.46141 E+01$

$2.46180 \mathrm{E}+01$

2. $462208+01$

2. $46261 \mathrm{E}+0$

$2.46301 \mathrm{E}+0$

$2.46341 E+0$

2. $46376 \mathrm{E}+0$

$2.46406 E+01$

2. $46436 \mathrm{E}+0$

$2.46471 \mathrm{E}+01$

2. $46517 \mathrm{E}+01$

$2.46572 \mathrm{E}+0$

$2.46635 E+01$
Xs 3

5. $00000 \mathrm{E}-01$

.00383E-0

5. $01905 \mathrm{E}-01$

5.04234 E-0

$5.06558 \mathrm{E}-01$

$5.08151 E-01$

5. $08720 \mathrm{E}-0$

.08477B-01

5.07964E-01

$5.07438 \mathrm{~B}-01$

$5.06914 \mathrm{E}-01$

5. 06313E-0

5.05589E-0

5. $04775 \mathrm{~B}-01$

$5.03963 \mathrm{~B}-01$

$5.03233 \mathrm{E}-0$

$5.02620 \mathrm{E}-01$

$5.02110 \mathrm{E}-01$

$5.01669 \mathrm{E}-01$

$5.01266 \mathrm{~B}-01$

$5.00891 \mathrm{E}-01$

$5.00546 \mathrm{E}-01$

$5.00233 \mathrm{~B}-01$

4. $99951 E-01$

4. $99700 \mathrm{E}-01$

$4.99475 \mathrm{~B}-01$

4. $99270 \mathrm{~B}-01$

$4.99078 \mathrm{~B}-01$

4. $98886 \mathrm{E}-0$

$4.98689 \mathrm{E}-01$

4. $98491 \mathrm{E}-01$

$4.98304 E-01$

$4.98143 \mathrm{E}-01$

$4.98015 E-01$

4. $97924 \mathrm{E}-01$

$4.97867 E-01$

$4.97836 \mathrm{E}-01$

4. $97821 \mathrm{E}-01$

$4.97817 \mathrm{E}-01$

4. $978178-01$

$4.97821 \mathrm{E}-0$

$4.97829 \mathrm{E}-0$

$4.97837 \mathrm{E}-01$

$4.97781 \mathrm{E}-01$

4. $97601 \mathrm{E}-01$

$4.97305 E-01$

4. $96953 \mathrm{E}-01$

4. $96624 \mathrm{z}-01$

4. $96370 \mathrm{E}-01$

4. $96209 \mathrm{E}-01$

4. $96136 \mathrm{E}-01$
ALF 3

8. 97215E-01

8. $97356 \mathrm{~B}-01$

8.97912E-01

$8.98757 \mathrm{E}-01$

8.99569E-01

$9.00070 \mathrm{E}-01$

$9.00153 \mathrm{E}-01$

$8.99909 \mathrm{E}-01$

8. $99560 \mathrm{E}-01$

8. $99216 \mathrm{E}-01$

8. $98890 \mathrm{~B}-01$

$8.98553 \mathrm{E}-01$

8. $98186 \mathrm{E}-01$

8. 97802 E-01

$8.97436 \mathrm{E}-01$

$8.97118 \mathrm{E}-01$

$8.96860 \mathrm{E}-01$

8.96657E-01

8. $96492 E-01$

8.96352E-01

$0.96230 \mathrm{~B}-01$

8. $96126 \mathrm{E}-01$

8.96039E-01

8. $95969 \mathrm{E}-01$

8.95914E-01

8. 95873E-01

8. $95841 \mathrm{E}-01$

Q. $95815 E-01$

$8.95791 \mathrm{E}-01$

8. $95766 \mathrm{~B}-01$

$8.95740 \mathrm{E}-01$

8.95720E-01

$8.95712 \mathrm{E}-01$

$8.95728 \mathrm{E}-01$

$8.95757 \mathrm{E}-01$

8.95793E-01

8. $95834 \mathrm{E}-01$

8. $95919 E-01$

$8.95960 \mathrm{E}-01$

8. $96038 \mathrm{E}-01$

8. $96051 \mathrm{E}-01$

$8.96015 \mathrm{E}-01$

$8.95748 \mathrm{E}-01$

8.95694E-01

8. $95680 \mathrm{E}-01$

$8.95701 \mathrm{p}-01$

HS 3

8. $98778 \mathrm{E}+02$

8. $98968 E+02$

$8.99722 E+02$

$.00876 E+02$

$9.02035 \mathrm{E}+02$

$9.02844 \mathrm{E}+02$

$9.03161 \mathrm{E}+02$

$9.03086 E+02$

$9.02880 E+02$

$9.02664 E+02$

$9.02445 E+02$

$9.02183 E+02$

$9.01855 \mathrm{E}+02$

$9.01479 E+02$

$.01098 E+02$

$9.00753 \mathrm{E}+02$

$9.00460 \mathrm{E}+02$

$9.00214 \mathrm{E}+02$

8. $99997 \mathrm{E}+02$

8. $99796 \mathrm{E}+02$

$8.99606 \mathrm{E}+02$

8. $99429 \mathrm{E}+02$

$8.99266 \mathrm{E}+02$

$8.99117 \mathrm{E}+02$

$8.98982 \mathrm{E}+02$ 
TIME

0.

$1.000005+00$

$2.00000 \mathrm{~F}+00$

$3.00000 \mathrm{E}+00$

$4.00000 \mathrm{E}+00$

$5.00000 \mathrm{E}+00$

$6.00000 E+00$

$7.00000 \mathrm{E}+00$

$8.00000 \mathrm{E}+00$

$9.00000 E+00$

$1.00000 E+01$

$1.10000 \mathrm{E}+01$

$1.20000 \Gamma+01$

$1.30000 E+01$

$1.40000 E+01$

$1.50000 \mathrm{E}+01$

$1.60000 \mathrm{E}+01$

$1.70000 \mathrm{E}+01$

$1.80000 \mathrm{E}+01$

1. $90000 E+01$

$2.00000 E+01$

2. $10000 E+01$

$2.20000 \mathrm{E}+01$

$2.30000 \mathrm{E}+01$

$2.40000 \mathrm{E}+01$

$2.50000 \mathrm{E}+01$

$2.60000 \mathrm{E}+01$

$2.70000 \mathrm{E}+01$

$2.80000 \mathrm{E}+01$

$2.90000 E+01$

$3.00000 \mathrm{E}+01$

$3.10000 E+01$

$3.20000 E+01$

3. $30000 E+01$

$3.40000 E+01$

$3.50000 \mathrm{E}+01$

$3.60000 \mathrm{E}+01$

$3.70000 E+01$

$3.80000 E+01$

3. $90000 E+01$

$4.00000 E+01$

4. $10000 \mathrm{E}+01$

$4.20000 E+01$

4. $30000 E+01$

$4.40000 E+01$

$4.50000 E+01$

4. $60000 \mathrm{E}+01$

4. $70000 \mathrm{E}+01$

$4.80000 \mathrm{~F}+01$

$4.90000 E+01$

$5.00000 E+01$
TI 8

7. $13367 \mathrm{E}+02$ 7. $13496 \mathrm{E}+02$ 7. $13875 E+02$ 7. $14228 \mathrm{E}+02$ $7.14439 E+02$ 7. $14537 \mathrm{E}+02$ 7. $14606 E+02$ 7. $14690 \mathrm{E}+02$ 7. $14800 \mathrm{E}+02$ 7. $14932 \mathrm{E}+02$ 7. $15071 \mathrm{E}+02$ 7. $15199 \mathrm{E}+02$ 7. $15301 \mathrm{E}+02$ 7. $15383 \mathrm{E}+02$ 7. $15452 E+02$ 7. $15510 E+02$ $7.15550 \mathrm{E}+02$ 7. $15568 \mathrm{E}+02$ 7. $15563 \mathrm{E}+02$ 7. $15536 E+02$ 7. 15490E + 02 7. $15430 \mathrm{E}+02$ $7.15358 \mathrm{E}+02$ 7. $15279 \mathrm{E}+02$ 7. $15196 E+02$ 7. $15109 \mathrm{E}+02$ 7. $15022 E+02$ 7. $14935 \mathrm{E}+02$ 7. $14851 \mathrm{E}+02$ $7.14771 \mathrm{E}+02$ 7. $14695 \mathrm{E}+02$ 7. $14619 E+02$ 7. $14542 \mathrm{E}+02$ 7. $14465 \mathrm{E}+02$ 7. $14386 \mathrm{E}+02$ $7.14307 \mathrm{E}+02$ 7. $14227 \mathrm{E}+02$ 7. $14146 E+02$ 7. $14066 E+02$ 7. $13987 \mathrm{E}+02$ 7. $13910 \mathrm{E}+02$ 7. $13834 \mathrm{E}+02$ 7. $13752 \mathrm{E}+02$ 7. $13693 E+02$ 7. $13624 \mathrm{E}+02$ $7.13548 \mathrm{E}+02$ $7.13463 \mathrm{E}+02$ 7. $13368 \mathrm{E}+02$ 7. $13266 \mathrm{E}+02$ 7. $13161 \mathrm{E}+02$ 7. $13057 F+02$
TI 9

$6.51000 \mathrm{E}+02$ $6.51065 \mathrm{~B}+02$ $6.51282 \mathrm{E}+02$ $6.51567 E+02$ $6.51867 \mathrm{E}+02$ $6.52152 E+02$ 6. $52406 \mathrm{E}+02$ $6.52618 \mathrm{~B}+02$ 6. $52776 \mathrm{E}+02$ $6.52879 E+02$ $6.52935 \mathrm{E}+02$ $6.52954 \mathrm{E}+02$ $6.52945 E+02$ $6.52916 \mathrm{E}+02$ $6.52877 \mathrm{E}+02$ $6.52833 E+02$ 6. $52788 \mathrm{E}+02$ $6.52742 E+02$ $6.52698 E+02$ $6.52653 \mathrm{E}+02$ $6.52606 \mathrm{E}+02$ $6.52558 \mathrm{E}+02$ $6.52505 E+02$ $6.52448 \mathrm{E}+02$ $6.52387 \mathrm{E}+02$ $6.52323 E+02$ $6.52257 \mathrm{E}+02$ $6.52188 \mathrm{E}+02$ $6.52118 \mathrm{E}+02$ $6.52046 \mathrm{E}+02$ $6.51972 \mathrm{E}+02$ $6.51897 \mathrm{E}+02$ $6.51819 \mathrm{E}+02$ $6.51742 \mathrm{E}+02$ $6.51667 \mathrm{E}+02$ $6.51594 \mathrm{E}+02$ $6.51525 E+02$ $6.51459 \mathrm{E}+02$ $6.51396 \mathrm{E}+02$ $6.51337 \mathrm{E}+02$ $6.51282 E+02$ $6.51229 \mathrm{E}+02$ $6.51180 \mathrm{E}+02$ $6.51134 \mathrm{E}+02$ $6.51091 \mathrm{E}+02$ $6.51050 E+02$ $6.51011 \mathrm{E}+02$ $6.50969 \mathrm{E}+02$ $6.50925 \mathrm{E}+02$ $6.50877 \mathrm{E}+02$

6. $50828 \mathrm{E}+0$
TAH 1

.58926E+02 7.38630E+02 $6.58975 \mathrm{E}+02$ $59160 \mathrm{E}+02$ $6.59160 \mathrm{E}+0$ . $59397 \mathrm{E}+02$ $6.59621 \mathrm{E}+02$ $6.59813 \mathrm{E}+02$ $6.59983 \mathrm{E}+02$ $6.60136 \mathrm{E}+02$ $6.60268 \mathrm{~B}+02$ $6.60377 \mathrm{E}+02$ $.60466 \mathrm{E}+02$ $6.60533 \mathrm{E}+02$ $6.60579 \mathrm{E}+02$ $6.60607 \mathrm{E}+02$ $6.60622 \mathrm{E}+02$ $6.606<6 \mathrm{E}+02$ $6.60615 E+02$ $6.60595 \mathrm{E}+02$ $6.60567 \mathrm{E}+02$ $6.60532 \mathrm{E}+02$ $6.60488 E+02$ $6.60439 \mathrm{E}+02$ $6.60384 \mathrm{E}+02$ $6.60324 \mathrm{E}+02$ $6.60262 E+02$ $6.60197 E+02$ $6.60131 \mathrm{E}+02$ . $60064 E+02$ $6.59997 \mathrm{E}+02$ $6.59930 \mathrm{E}+02$ $6.59862 \mathrm{E}+02$ $6.59794 \mathrm{E}+02$ $6.59726 E+02$ $6.59660 E+02$ $6.59594 E+02$ $6.59531 \mathrm{E}+02$ $6.59470 \mathrm{E}+02$ $6.59411 \mathrm{E}+02$ $6.59355 E+02$ $6.59301 \mathrm{E}+02$ $6.59249 \mathrm{E}+02$ $6.59201 \mathrm{E}+02$ $6.59155 \mathrm{E}+02$ $6.59111 \mathrm{E}+02$ $6.59068 E+02$ $6.59022 \mathrm{E}+02$ $6.58971 \mathrm{E}+02$ $6.58915 \mathrm{E}+02$ $6.58856 \mathrm{E}+02$ $6.58794 \mathrm{E}+02$
HS 1

$5.42398 \mathrm{E}+02$ $5.42398 \mathrm{E}+02$ $5.42398 \mathrm{E}+02$ $5.42398 E+02$ $5.42398 \mathrm{E}+02$ $5.42398 \mathrm{E}+02$ $5.42398 \mathrm{E}+02$ $5.42398 \mathrm{E}+02$ $5.42398 E+02$ $5.42398 E+02$ $5.42398 \mathrm{E}+02$ $5.42398 E+02$ $5.42398 \mathrm{E}+02$ $5.42398 \mathrm{E}+02$ $5.42398 \mathrm{E}+02$ $5.42398 \mathrm{E}+02$ $5.42398 \mathrm{E}+02$ $5.42398 k+02$ $5.42398 \mathrm{E}+02$ $5.42398 B+02$ $5.42398 \mathrm{E}+02$ $5.42398 \mathrm{E}+02$ $5.42398 E+02$ $5.42398 E+02$ $5.42398 \mathrm{E}+02$ $5.42397 \mathrm{E}+02$ $5.42393 \mathrm{E}+02$ $5.42378 \mathrm{E}+02$ $5.42350 E+02$ $5.42313 E+02$ $5.42276 E+02$ $5.42249 E+02$ $5.42233 \mathrm{E}+02$ $5.42229 E+02$ $5.42234 \mathrm{E}+02$ $5.42247 \mathrm{E}+02$ $5.42268 E+02$ $5.42296 E+02$ $5.42330 \mathrm{E}+02$ $5.42368 E+02$ $5.42410 \mathrm{E}+02$ $5.42452 E+02$ $5.42495 \mathrm{E}+02$ $5.42538 \mathrm{E}+02$ $5.42580 \mathrm{E}+02$ $5.42621 E+02$ $5.42660 \mathrm{E}+02$ $5.42697 E+02$ $5.42732 \mathrm{E}+02$ $5.42764 E+02$ $5.42794 \mathrm{~F}+02$

TSAT

$6.22415 \mathrm{E}+02$ $6.22415 E+02$ $6.22417 \mathrm{E}+0$ 6. $22423 E+02$ $6.22450 \mathrm{E}+02$ $6.22516 \mathrm{E}+02$ $6.22624 E+02$ $6.22762 E+02$ $6.22907 \mathrm{E}+02$ $6.23042 E+02$ $6.23161 \mathrm{E}+02$ $6.23264 \mathrm{E}+02$ $6.23353 \mathrm{E}+02$ $6.23427 E+02$ $6.23486 \mathrm{E}+02$ $6.23528 E+02$ $6.23554 \mathrm{E}+02$ $6.23566 \mathrm{E}+02$ $6.23567 \mathrm{E}+02$ $6.23559 \mathrm{E}+02$ $6.23545 E+02$ $6.23524 \mathrm{E}+02$ . $23498 E+02$ $6.23469 \mathrm{E}+02$ 


\begin{tabular}{|c|c|c|c|c|}
\hline $\begin{array}{l}\text { TIME } \\
0 . \\
1.00000 E+00 \\
2.00000 E+00 \\
3.00000 E+00 \\
4.00000 E+00 \\
5.00000 E+00 \\
6.00000 E+00 \\
7.00000 E+00 \\
8.00000 E+00 \\
9.00000 E+00 \\
1.00000 E+01 \\
1.10000 E+01 \\
1.20000 E+01 \\
1.30000 E+01 \\
1.40000 E+01 \\
1.50000 E+01 \\
1.60000 E+01 \\
1.70000 E+01 \\
1.80000 E+01 \\
1.90000 E+01 \\
2.00000 E+01 \\
2.10000 E+01 \\
2.20000 E+01 \\
2.30000 E+01 \\
2.40000 E+01 \\
2.50000 E+01 \\
2.60000 E+01 \\
2.70000 E+01 \\
2.80000 E+01 \\
2.90000 E+01 \\
3.00000 E+01 \\
3.10000 E+01 \\
3.20000 E+01 \\
3.30000 E+01 \\
3.40000 E+01 \\
3.50000 E+01 \\
3.60000 E+01 \\
3.70000 E+01 \\
3.80000 E+01 \\
3.90000 E+01 \\
4.00000 E+01 \\
4.10000 E+01 \\
4.20000 E+01 \\
4.30000 E+01 \\
4.40000 E+01 \\
4.50000 E+01 \\
4.60000 E+01 \\
4.70000 E+01 \\
4.80000 F+01 \\
4.90000 E+01 \\
5.00000 E+01\end{array}$ & $\begin{array}{c}\text { HS } \\
6.70821 E+06 \\
6.70753 E+06 \\
6.70375 E+06 \\
6.69913 E+06 \\
6.69717 E+06 \\
6.69908 E+06 \\
6.70378 E+06 \\
6.70903 E+06 \\
6.71280 E+06 \\
6.71527 E+06 \\
6.71724 E+06 \\
6.71916 E+06 \\
6.72112 E+06 \\
6.72290 E+06 \\
6.72422 E+06 \\
6.72497 E+06 \\
6.72527 E+06 \\
6.72529 E+06 \\
6.72520 E+06 \\
6.72508 E+06 \\
6.72492 E+06 \\
6.72469 E+06 \\
6.72439 E+06 \\
6.72404 E+06 \\
6.72364 E+06 \\
6.72322 E+06 \\
6.72280 E+06 \\
6.72239 E+06 \\
6.72203 E+06 \\
6.72171 E+06 \\
6.72139 E+06 \\
6.72102 E+06 \\
6.72057 E+06 \\
6.72006 E+06 \\
6.71950 E+06 \\
6.71893 E+06 \\
6.71837 E+06 \\
6.71783 E+06 \\
6.71734 E+06 \\
6.71687 E+06 \\
6.71643 E+06 \\
6.71598 E+06 \\
6.71555 E+06 \\
6.71527 E+06 \\
6.71522 E+06 \\
6.71527 E+06 \\
6.71518 E+06 \\
6.71482 E+06 \\
6.71419 E+06 \\
6.71340 E+06 \\
6.71255 E+06\end{array}$ & $\begin{array}{c}153 \\
6.70821 E+06 \\
6.70781 E+06 \\
6.70579 E+06 \\
6.69992 E+06 \\
6.69105 E+06 \\
6.68259 E+06 \\
6.67783 E+06 \\
6.67816 E+06 \\
6.68232 E+06 \\
6.68782 E+06 \\
5.69319 E+06 \\
6.69822 E+06 \\
6.70312 E+06 \\
6.70802 E+06 \\
6.71282 E+06 \\
6.71719 E+06 \\
6.72082 E+06 \\
6.72362 E+06 \\
6.72569 E+06 \\
6.72723 E+06 \\
6.72840 E+06 \\
6.72929 E+06 \\
6.72994 E+06 \\
6.73037 E+06 \\
6.73060 E+06 \\
6.73065 E+06 \\
6.73056 E+06 \\
6.73037 E+06 \\
6.73011 E+06 \\
6.72986 E+06 \\
6.72963 E+06 \\
6.72940 E+06 \\
6.72911 E+06 \\
6.72871 E+06 \\
6.72817 E+06 \\
6.72749 E+06 \\
6.72670 E+06 \\
6.72584 E+06 \\
6.72495 E+06 \\
6.72407 E+06 \\
6.72323 E+06 \\
6.72243 E+06 \\
6.72168 E+06 \\
6.72103 E+06 \\
6.72064 E+06 \\
6.72068 E+06 \\
6.72110 E+06 \\
6.72163 E+06 \\
6.72199 E+06 \\
6.72198 E+06 \\
6.72156 E+06\end{array}$ & 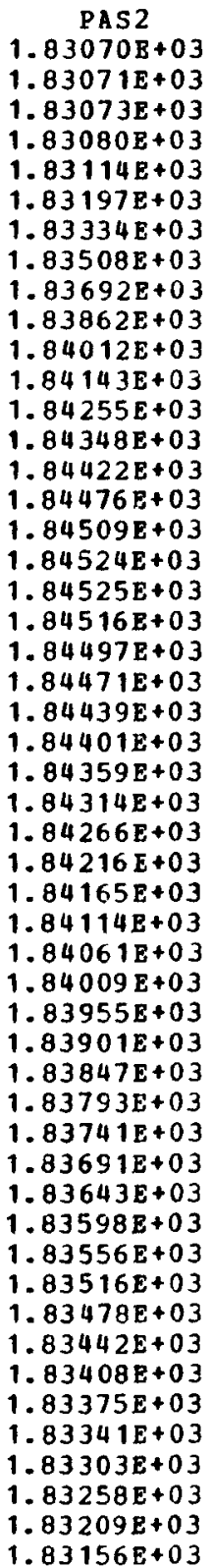 & $\begin{array}{l}\mathrm{ALFA2} \\
6.14591 \mathrm{E}-01 \\
6.14720 \mathrm{E}-01 \\
6.15233 \mathrm{E}-01 \\
6.16011 \mathrm{E}-01 \\
6.16752 \mathrm{E}-01 \\
6.17192 \mathrm{E}-01 \\
6.17231 \mathrm{E}-01 \\
6.16958 \mathrm{E}-01 \\
6.16584 \mathrm{E}-01 \\
6.16219 \mathrm{E}-01 \\
6.15876 \mathrm{E}-01 \\
6.15529 \mathrm{E}-01 \\
6.15159 \mathrm{E}-01 \\
6.14779 \mathrm{E}-01 \\
6.14420 \mathrm{E}-01 \\
6.14113 \mathrm{E}-01 \\
6.13866 \mathrm{E}-01 \\
6.13675 \mathrm{E}-01 \\
6.13523 \mathrm{E}-01 \\
6.13396 \mathrm{E}-01 \\
6.13289 \mathrm{E}-01 \\
6.13200 \mathrm{E}-01 \\
6.13129 \mathrm{E}-01 \\
6.13076 \mathrm{E}-01 \\
6.13037 \mathrm{E}-01 \\
6.13011 \mathrm{E}-01 \\
6.12995 \mathrm{E}-01 \\
6.12985 \mathrm{E}-01 \\
6.12977 \mathrm{E}-01 \\
6.12968 \mathrm{E}-01 \\
6.12959 \mathrm{E}-01 \\
6.12955 \mathrm{E}-01 \\
6.12960 \mathrm{E}-01 \\
6.12977 \mathrm{E}-01 \\
6.13007 \mathrm{E}-01 \\
6.13048 \mathrm{E}-01 \\
6.13096 \mathrm{E}-01 \\
6.13148 \mathrm{E}-01 \\
6.13200 \mathrm{E}-01 \\
6.13251 \mathrm{E}-01 \\
6.13300 \mathrm{E}-01 \\
6.13348 \mathrm{E}-01 \\
6.13394 \mathrm{E}-01 \\
6.13415 \mathrm{E}-01 \\
6.13392 \mathrm{E}-01 \\
6.13326 \mathrm{E}-01 \\
6.13244 \mathrm{E}-01 \\
6.13174 \mathrm{E}-01 \\
6.13137 \mathrm{E}-01 \\
6.13137 \mathrm{E}-01 \\
6.13171 \mathrm{E}-01\end{array}$ \\
\hline
\end{tabular}




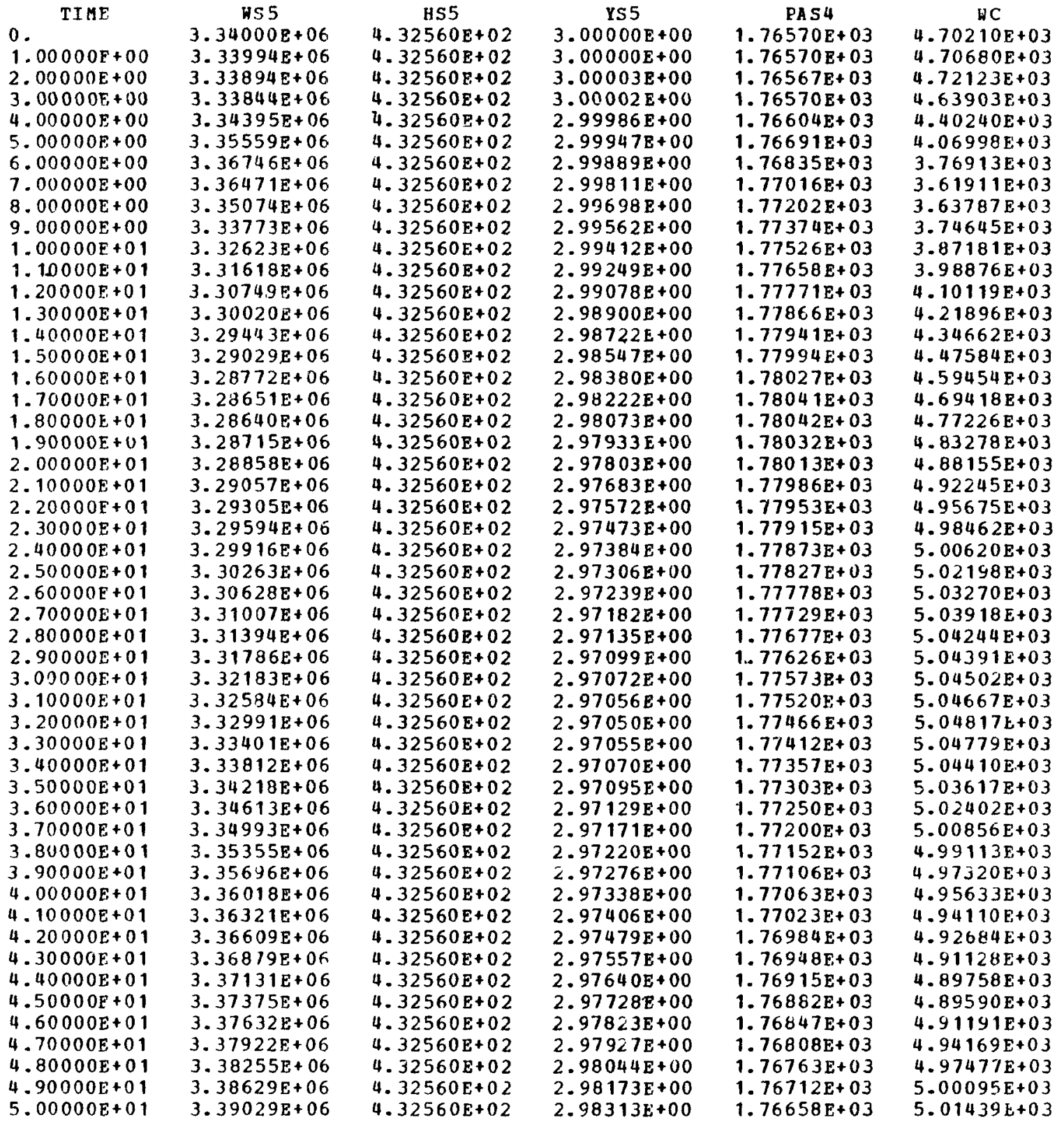

EM S4

2. $17993 E+03$ $2.17993 \mathrm{E}+0$ 2. $17987 \mathrm{E}+03$ 2. $17992 E+03$ 2. $18056 E+03$ 2. $18222 \mathrm{E}+03$ 2. $18492 \mathrm{E}+03$ 2. $18835 \mathrm{E}+0$ 2. $19205 E+03$ 2. $19562 \mathrm{E}+03$ 2. $19891 \mathrm{E}+03$ $2.20193 \mathrm{E}+03$ . $20469 \mathrm{E}+0$ $2.20715 \mathrm{E}+0$ $2.20927 \mathrm{E}+03$ 2. $21101 \mathrm{E}+03$ $2.21235 \mathrm{E}+03$ $2.21335 E+03$ $2.21406 E+03$ $2.21453 \mathrm{E}+03$ 2. $21481 \mathrm{E}+0$ $2.21491 \mathrm{E}+03$ $2.21486 E+03$ $2.21466 \pm+03$ $2.21391 E+0$ 2. $21339 E+03$ 2. $21280 \mathrm{E}+03$ 2. $21213 \mathrm{E}+03$ $2.21141 \mathrm{E}+03$ 2. $21063 E+03$ $2.20979 \mathrm{E}+0$ 2. $20889 \mathrm{E}+03$ $2.20793 \mathrm{E}+03$ $2.20692 \mathrm{E}+03$ $2.20588 \mathrm{E}+03$ $2.20482 E+03$ 2. $20376 \mathrm{E}+03$ $2.20270 E+03$ 2. $20167 \mathrm{E}+0 \mathrm{~J}$ $2.20065 E+03$ 2. $19964 \mathrm{E}+03$ 2. $19864 E+03$ 2. $19767 \mathrm{E}+03$ 2. $19671 E+03$ $2.19574 E+03$ 2. $19471 E+0$ 2. $19356 \mathrm{E}+03$ 2. 19225E+0 2. $19079 E+03$ 2. $18922 \mathrm{E}+03$
EMS 5

2. $34510 E+04$ 2. $34510 E+04$ $2.34511 \mathrm{E}+04$ 2. $34511 E+04$ $2.34503 E+04$ 2. $34484 \mathrm{E}+04$ $2.34455 E+04$ 2. $34416 \mathrm{E}+04$ 2. $34360 \mathrm{E}+04$ 2. $34292 \mathrm{E}+04$ 2. $34217 \mathrm{E}+04$ $2.34136 E+04$ 2. $34051 E+04$ $2.33963 E+04$ 2. $33874 \mathrm{E}+04$ $2.33787 \mathrm{E}+04$ 2. $33704 E+04$ 2. $33625 E+04$ 2. $33551 E+04$ 2. $33481 E+04$ $2.33417 \mathrm{E}+04$ 2. $33357 \mathrm{E}+04$ 2. $33302 \mathrm{E}+04$ 2. $33252 E+04$ 2. $33208 E+04$ 2. $33169 E+04$ 2. $33136 E+04$ 2. $33108 E+04$ 2. $33084 \mathrm{E}+04$ 2. $33066 E+04$ 2. $33053 E+04$ 2. $33045 E+04$ 2. $33042 \mathrm{E}+04$ 2. $33045 E+04$ 2. $33052 \mathrm{E}+04$ 2. $33064 \mathrm{E}+04$ $2.33081 E+04$ 2. $33102 E+04$ 2. $33126 E+04$ 2. $33154 \mathrm{E}+04$ 2. $33185 E+04$ 2. $33219 E+04$ 2. $33255 E+04$ 2. $33294 E+04$ $2.33335 \mathrm{E}+04$ $2.33379 E+04$ 2. $33426 E+04$ 2. $33479 \mathrm{E}+04$ $2.33537 \mathrm{E}+04$ $2.33601 \mathrm{E}+0 \mathrm{II}$ 2. $33671 \mathrm{E}+04$ 
LIST,TP7,TI3,TP5,TI1,TAP5, TAI1

TIME

$1.00000 \mathrm{E}+00$

2. $00000 \mathrm{E}+00$

$3.00000 E+00$

$4.00000 E+00$

$5.00000 \mathrm{E}+00$

$6.00000 \mathrm{E}+00$

$7.00000 \mathrm{E}+00$

$8.00000 \mathrm{E}+00$

$9.00000 \mathrm{x}+00$

$1.00000 E+01$

$1.10000 \mathrm{E}+01$

$1.20000 E+01$

1. $30000 E+01$

1. $40000 E+01$

1. $50000 \mathrm{E}+01$

1. $60000 \mathrm{E}+01$

$1.70000 \mathrm{E}+01$

$1.80000 E+01$

$1.90000 E+01$

$2.000008+01$

2. $10000 \mathrm{E}+01$

2. $20000 E+01$

$2.30000 E+01$

$2.40000 E+01$

$2.50000 E+01$

$2.60000 \mathrm{E}+01$

$2.70000 \mathrm{E}+01$

2. $80000 E+01$

2. $90000 \mathrm{E}+01$

$3.00000 E+01$

$3.10000 E+01$

$3.20000 \mathrm{E}+01$

$3.30000 \mathrm{E}+01$

$3.40000 E+01$

3. $50000 \mathrm{E}+01$

$3.60000 \mathrm{E}+01$

$3.70000 \mathrm{E}+01$

$3.80000 E+01$

3. $90000 \mathrm{E}+01$

$4.00000 \mathrm{E}+01$

4. 10000E+01

4. $20000 E+01$

4. $30000 E+01$

$4.40000 E+01$

$4.50000 \mathrm{E}+01$

$4.60000 E+01$

4. $70000 E+01$

4. $80000 \mathrm{E}+01$

4. $90000 E+01$

$5.00000 \mathrm{E}+01$
TP 7

TI 3

$.30000 \mathrm{E}+02$

7. $30861 \mathrm{E}+02$

7. $31029 E+02$

7. $30948 \mathrm{E}+02$

7. $30803 \mathrm{E}+02$

7. $30672 E+02$,

7. $30562 \mathrm{E}+02$

7. $30465 E+02$

7. $30369 \mathrm{E}+02$

7. $30267 E+02$

. $30158 \mathrm{E}+02$

$7.30045 \mathrm{E}+02$

7. $29956 \mathrm{E}+02$

$7.29898 \mathrm{E}+02$

$29860 E+02$

$7.29830 \mathrm{E}+02$

7. $29803 \mathrm{E}+02$

$7.29778 \mathrm{E}+02$

$7.29757 \mathrm{E}+02$

$7.29740 E+02$

$7.29728 \mathrm{E}+02$

$29730 \mathrm{E}+02$

7. $29771 \mathrm{E}+02$

$7.29857 \mathrm{E}+02$

$7.29978 \mathrm{E}+02$

7. $30122 \mathrm{E}+02$

7. $30280 \mathrm{E}+02$

7. $30442 \mathrm{E}+02$

7. $30598 \mathrm{E}+02$

7. $30740 \mathrm{E}+02$

7. $30865 \mathrm{E}+02$

7. $30970 \mathrm{E}+02$

$7.31055 E+02$

$31120 E+02$

7. $31166 \mathrm{E}+02$

7. $31195 \mathrm{E}+02$

7. $31210 E+02$

7. $31212 E+02$

7. $31203 E+02$

. $31183 E+02$

$7.31154 E+02$

7. $31116 \mathrm{E}+02$

7. $31069 \mathrm{E}+02$

7. $31014 \mathrm{E}+02$

7. $30950 E+02$

7. $30878 \mathrm{E}+02$

7. $30713 \mathrm{E}+02$

7. $30621 \mathrm{E}+02$

7. $30524 E+02$
9. $36000 \mathrm{E}+02$

$9.35804 E+02$

$9.35276 \mathrm{E}+02$

. $34811 E+02$

. $34568 \mathrm{E}+02$

. $34532 \mathrm{~F}+02$

9. $34589 \mathrm{E}+02$

9. $34663 \mathrm{E}+02$

$9.34731 \mathrm{E}+02$

. $34797 B+02$

9. $34870 \mathrm{E}+02$

. $34953 E+02$

$9.35048 E+02$

9. $35136 \mathrm{E}+02$

$9.35198 \mathrm{E}+02$

$35234 \mathrm{E}+02$

. $35259 E+02$

. $35289 \mathrm{E}+02$

9. $35328 \mathrm{E}+02$

9. $35377 \mathrm{E}+02$

. $35433 E+02$

$35494 E+02$

$35554 \mathrm{E}+02$

9. $35595 E+02$

$9.35627 \mathrm{E}+02$

9. $35662 \mathrm{E}+02$

$9.35708 E+02$

9. $35767 \mathrm{E}+02$

9. $35839 \mathrm{E}+02$

9. $35923 \mathrm{E}+02$

9. $36016 E+02$

. $36114 \mathrm{E}+02$

. $36213 E+02$

. $36308 E+02$

$9.36394 \mathrm{E}+02$

$36469 E+02$

. 3653

. $36590 \mathrm{E}+02$

. $36634 \mathrm{E}+02$

$9.36667 \mathrm{E}+02$

9. $36691 \mathrm{E}+02$

9. $36705 E+02$

9. $36711 \mathrm{E}+02$

. $36709 E+02$

$.36701 E+02$

$9.36685 \mathrm{E}+02$

9. $36663 \mathrm{E}+02$

9. $36636 \mathrm{E}+02$

9. $36603 E+02$

$9.36565 E+02$

9. $36522 \mathrm{E}+02$
IP5

$9.94731 \mathrm{E}+02$

$9.947318+02$

$9.94731 \mathrm{E}+02$

$9.947318+02$

$9.94731 \mathrm{E}+02$

$9.94731 \mathrm{E}+02$

$9.94731 \mathrm{E}+02$

$9.94731 \mathrm{E}+02$

$9.94731 \mathrm{E}+02$

$9.94731 \mathrm{E}+02$

$9.947318+02$

$9.94731 \mathrm{E}+02$

$9.94731 \mathrm{E}+02$

$9.94731 \mathrm{E}+02$

$9.94731 \mathrm{E}+02$

$9.94730 \mathrm{E}+02$

$9.94750 B+02$

$9.94791 E+02$

$9.94840 \mathrm{E}+02$

$9.94893 \mathrm{E}+02$

$9.94948 E+02$

$9.95003 E+02$

$95056 \mathrm{E}+02$

$9.95106 \mathrm{E}+02$

$9.95152 \mathrm{E}+02$

$9.95192 \mathrm{E}+02$

$9.95226 E+02$

$9.95253 \mathrm{E}+02$

$9.95273 E+02$

$9.95288 E+02$

$9.95299 E+02$

$9.95305 E+02$

$9.95308 \mathrm{E}+02$

$9.95308 \mathrm{E}+02$

$9.95305 \mathrm{E}+02$

$9.95299 \mathrm{E}+02$

$9.95291 \mathrm{E}+02$

$9.95280 \mathrm{E}+02$

$9.95269 E+02$

$9.95255 \mathrm{E}+02$

$9.95240 \mathrm{E}+02$

9. $95206 \mathrm{E}+02$

$9.95188 \mathrm{E}+02$

$9.95169 E+02$

$9.95150 E+02$

$9.95129 \mathrm{E}+02$

$9.95109 E+02$

$9.95067 E+02$

$9.95046 \mathrm{E}+02$
TI1

$6.51000 \mathrm{E}+02$

$6.51000 \mathrm{E}+02$

$6.51000 \mathrm{E}+02$

$6.51000 E+02$

$6.51000 \mathrm{E}+02$

$6.51000 \mathrm{E}+02$

$6.51000 E+02$

$6.51000 \mathrm{E}+02$

$6.51000 \mathrm{E}+02$

$6.51000 \mathrm{E}+02$

$6.51000 E+02$

$6.51000 \mathrm{E}+02$

$6.51000 \mathrm{E}+02$

$6.51000 E+02$

$6.51000 \mathrm{E}+02$

$6.51000 \mathrm{E}+02$

$6.51000 \mathrm{E}+02$

$6.51000 \mathrm{E}+02$

$6.51000 \mathrm{E}+02$

$6.51000 E+02$

$6.51000 E+02$

$6.51003 E+02$

$6.51094 \mathrm{E}+02$

$6.51319 \mathrm{E}+02$

$6.51601 \mathrm{E}+02$

$6.51893 \mathrm{E}+02$

$6.52168 E+02$

$6.52415 \mathrm{E}+02$

$6.52621 \mathrm{E}+02$

$6.52775 \mathrm{E}+02$

$6.52877 \mathrm{E}+02$

$6.52933 \mathrm{E}+02$

$6.52953 \mathrm{E}+02$

$6.52946 \mathrm{E}+02$

$6.52918 E+02$

$6.52880 \mathrm{E}+02$

$6.52837 \mathrm{E}+02$

$6.52792 E+02$

$6.52747 \mathrm{E}+02$

$6.52703 E+02$

$6.52658 \mathrm{E}+02$

$6.52613 \mathrm{E}+02$

6. $52565 \mathrm{E}+02$

$6.52513 \mathrm{E}+02$

$6.52457 E+02$

$6.52398 \mathrm{E}+02$

$6.52335 E+02$

$6.52201 \mathrm{E}+02$

6. $52061 \mathrm{E}+02$

TAP 5

$8.62366 E+02$

8. $62530 \mathrm{E}+02$

$62796 \mathrm{E}+02$

$628000+02$

8. $62839 E+02$

. $62767 \mathrm{E}+02$

$8.62702 \mathrm{E}+02$

$8.62647 \mathrm{E}+02$

$.62598 E+02$

$62550 \mathrm{E}+02$

$62445 E+02$

$8.62388 \mathrm{E}+02$

$8.62344 E+02$

$.62315 E+02$

$62295 \mathrm{E}+02$

8. $62290 \mathrm{E}+02$

82297E+02

8. $62309 \mathrm{E}+02$

8. $62325 F+02$

$8.62344 E+02$

$62365 \mathrm{~F}+02$

8. $62393 \mathrm{E}+$

. $62438 \mathrm{E}+02$

$.62504 E+02$

8. $62585 E+02$ 
TIMF,

1. $00000 \mathrm{E}+00$

2. $00000 E+00$

3. $00000 \mathrm{E}+00$

$3.00000 \mathrm{E}+00$

$4.00000 \mathrm{E}+00$

$5.00000 \mathrm{E}+00$
$6.00000 \mathrm{~F}+00$

$7.00000 \mathrm{E}+00$

8. $00000 \mathrm{E}+00$

$9.00000 \mathrm{E}+00$

1. $00000 \mathrm{E}+01$

1. $10000 \mathrm{E}+01$

$1.20000 F+01$

1. $30000 \mathrm{E}+01$

$1.40000 \mathrm{E}+01$

$1.50000 \mathrm{E}+01$

$1.60000 E+01$

$1.70000 \mathrm{E}+01$

$1.80000 \mathrm{E}+01$

1. $90000 E+01$

$2.00000 \mathrm{E}+01$

2. $10000 \mathrm{E}+01$

$2.20000 E+01$

$2.30000 \mathrm{E}+01$

$2.40000 \mathrm{E}+01$

$2.50000 \mathrm{E}+01$

$2.60000 E+01$

$2.70000 \mathrm{E}+01$

$2.80000 \mathrm{E}+01$

$2.90000 \mathrm{E}+01$

$3.00000 \mathrm{E}+01$

3. $10000 \mathrm{E}+01$

$3.20000 E+01$

$3.30000 \mathrm{E}+01$

$3.40000 \mathrm{E}+01$

$3.50000 E+01$

$3.60000 \mathrm{E}+01$

3. $70000 \mathrm{E}+01$

$3.80000 \mathrm{E}+01$

$3.9000 O E+01$

4. $00000 \mathrm{E}+01$

4. $10000 E+01$

4. $20000 E+01$

4. $30000 \mathrm{E}+01$

$4.40000 E+01$

$4.50000 \mathrm{E}+01$

$4.60000 E+01$

$4.70000 \mathrm{E}+01$

4. $80000 E+01$

4. $90000 E+0$

$5.00000 E+01$
NP
4. $14460 \mathrm{E}+07$

4. $23798 \mathrm{E}+07$

4. $26252 \mathrm{E}+07$

4. $25020 E+07$

4. $23869 E+07$

4. $23023 \mathrm{E}+07$

$4.22302 \mathrm{E}+07$

4. $21603 \mathrm{E}+07$

4. $20868 E+07$

4. $200498+07$

4. $19121 \mathrm{E}+07$

4. $18099 \mathrm{E}+07$

4. $17196 \mathrm{E}+07$

4. $16740 E+07$

4. $16480 E+07$

4. $16286 E+07$

4. $16111 E+07$

4. $15945 E+07$

4. $15779 E+07$

4. $15615 E+07$

4. $15458 \varepsilon+07$

4. $15311 \mathrm{E}+07$

4. $15173 \mathrm{E}+07$

4. $15043 \mathrm{E}+07$

4. $14918 \mathrm{E}+07$

4. $14800 \mathrm{E}+07$

4. $14687 \mathrm{E}+07$

4. $14580 E+07$

4. $14478 E+07$

4. $14376 \mathrm{E}+07$

4. 14280E+ 07

4. $14187 \mathrm{E}+07$

4. $14096 \mathrm{E}+07$

4. $14006 E+07$

4. $13910 \mathrm{E}+07$

4. $13807 E+07$

4. $13684 \mathrm{E}+07$

4. 13560E+07

4. $13411 \mathrm{E}+07$

4. $13079 \mathrm{E}+07$

4. $12900 \mathrm{E}+07$

4. $12714 \mathrm{E}+07$

4. $12523 E+07$

4. 12327E+07

4. $12124 \mathrm{E}+07$

4. $11918 \mathrm{E}+07$

4. $11712 \mathrm{E}+07$

4. $11507 \mathrm{E}+07$

4. $11302 E+07$

4. $11098 E+07$
HI

3. $83400 \mathrm{E}+07$

$3.88615 E+07$

$.93473 E+07$

3. $95033 \mathrm{E}+07$

3. $94481 E+07$

3. $93292 E+07$

$3.92312 E+07$

3. $91572 E+07$

$3.90913 E+07$

$3.90216 \mathrm{E}+07$

$3.89426 \mathrm{E}+07$

3. $88534 \mathrm{E}+07$

3. $87627 \mathrm{E}+07$

$3.87017 \mathrm{E}+07$

$3.86685 \mathrm{E}+07$

$3.86490 \mathrm{E}+07$

3. $86333 \mathrm{E}+07$

3. $86175 \mathrm{E}+07$

$3.86003 E+07$

3. $85818 \mathrm{E}+07$

3. $85626 E+07$

$3.854338+07$

3. $85242 \mathrm{E}+07$

3.85054E+07

$3.84868 \mathrm{E}+07$

3. $84686 E+07$

$3.84508 E+07$

$3.84332 \mathrm{E}+0 \mathrm{~T}$

3. $84161 \mathrm{E}+07$

3. $83993 \mathrm{E}+07$

3. $83828 \mathrm{E}+07$

$3.83667 \mathrm{E}+07$

. $83512 E+07$

$3.83385 \mathrm{E}+07$

3. $83269 \mathrm{E}+07$

$3.83153 \mathrm{E}+07$

$3.83033 \mathrm{E}+07$

3. $82915 \mathrm{E}+07$

$3.82798 \mathrm{E}+07$

3. $82685 E+07$

$3.82576 \mathrm{E}+07$

3. $82473 \mathrm{E}+07$

$3.82377 \mathrm{E}+07$

$3.82288 \mathrm{E}+07$

$3.82206 \mathrm{E}+07$

$3.82130 E+07$

3. $82057 \mathrm{E}+07$

3. $81990 \mathrm{E}+07$

3. $81927 \mathrm{E}+07$

$3.81870 \mathrm{E}+07$

3. $81818 \mathrm{E}+07$ $x$

1. $20000 \mathrm{E}+01$

1. $18500 E+01$

1. $17000 \mathrm{E}+01$

1. $15500 \mathrm{E}+01$

$14000 \mathrm{E}+01$

$1.12500 \mathrm{E}+01$

$1.11000 E+01$

1. $09500 \mathrm{E}+01$

$.08000 \mathrm{E}+0$

$1.05000 \mathrm{E}+01$

1. $03500 \mathrm{E}+01$

$1.02913 \mathrm{E}+01$

$1.02650 \mathrm{E}+01$

$.02528 \mathrm{E}+01$

$1.02458 E+01$

1. $02458 \mathrm{E}+01$

1. $02458 \mathrm{E}+01$

$1.02458 \mathrm{E}+01$

$1.02458 E+01$

$.02458 \mathrm{E}+01$

$1.02458 \mathrm{E}+01$

$1.02458 \mathrm{E}+01$

$1.02458 \mathrm{E}+01$

$1.02458 \mathrm{E}+01$

$1.02458 \mathrm{E}+01$

$.02458 \mathrm{E}+01$

$1.02458 \mathrm{E}+01$

$1.02458 \mathrm{E}+01$

$1.02458 \mathrm{E}+01$

$1.02458 \mathrm{E}+01$

$1.02458 \mathrm{E}+01$

$.02474 \mathrm{E}+01$

$1.02501 \mathrm{E}+01$

$1.02549 E+01$

$1.02571 \mathrm{E}+01$

$1.02603 \mathrm{E}+01$

$1.02646 E+01$

$.02694 \mathrm{E}+01$

$1.02732 \mathrm{E}+01$

$1.02774 \mathrm{E}+01$

$1.02823 \mathrm{E}+01$

$1.02876 \mathrm{E}+01$

1. $02930 E+01$

$.02962 \mathrm{E}+01$

$1.03007 \mathrm{E}+01$

$1.03058 \mathrm{E}+01$

$1.03093 E+01$

$1.03130 \mathrm{E}+01$

$1.03165 \mathrm{E}+01$

$1.03198 \mathrm{E}+01$
RHOD

9.66686E-09

$4.60804 \mathrm{E}-02$

2. $31921 \mathrm{E}-02$

1. $28016 \mathrm{E}-02$

$7.04656 \mathrm{E}-03$

$3.04528 \mathrm{E}-03$

$-2.21536 \mathrm{E}-04$

$3.10828 \mathrm{E}-03$

$-5.76897 \mathrm{E}-03$

$-8.29444 \mathrm{E}-03$

$-1.06727 \mathrm{E}-02$

$-1.28957 \mathrm{E}-02$

$-1.06112 \mathrm{E}-02$

$.64165 \mathrm{E}-03$

$7.21430 \mathrm{E}-03$

$-6.31606 \mathrm{E}-03$

$-5.47243 \mathrm{E}-03$

$-4.92140 \mathrm{E}-03$

$-4.50816 \mathrm{E}-03$

$-4.16795 E-03$

$-3.61292 \mathrm{E}-03$

$-3.38738 \mathrm{E}-03$

$-3.18852 \mathrm{E}-0$

$-3.00642 \mathrm{E}-03$

$2.84383 \mathrm{E}-03$

$2.69675 \mathrm{E}-03$

$-2.56249 \mathrm{E}-03$

$-2.44076 \mathrm{E}-03$

$-2.32900 \mathrm{E}-03$

$-2.23036 \mathrm{E}-03$

$2.14686 \mathrm{E}-03$

$-2.00657 E-03$

$-1.86243 \mathrm{E}-03$

$-1.63162 \mathrm{E}-03$

$-1.56308 \mathrm{E}-0$

. $50654 \mathrm{E}-03$

$-1.39583 \mathrm{E}-03$

$-1.36625 E-03$

$-1.30800 \mathrm{E}-0$

$-1.23628 \mathrm{E}-03$

- 1. $14278 \mathrm{E}-03$

$1.05659 \mathrm{E}-03$

$-84327 \mathrm{E}-04$

$80 \mathrm{E}-04$

$-8.23877 \mathrm{E}-04$

EN

$1.00000 E+00$

$1.07770 \mathrm{E}+00$

$1.05670 \mathrm{E}+00$

$1.04532 E+00$

$1.03793 E+00$

$.03196 \mathrm{E}+00$

$1.02640 \mathrm{E}+00$

$1.02089 \mathrm{E}+00$

$1.01532 \mathrm{E}+00$

$1.00959 \mathrm{E}+00$

$1.00377 \mathrm{E}+00$

9.97909 E-0

9.97165E-01

$9.97237 \mathrm{E}-0$

$9.97302 \mathrm{E}-01$

$9.97173 E-0$

$9.97193 \mathrm{E}-01$

$9.97080 \mathrm{E}-01$

$9.96928 \mathrm{E}-01$

$9.96779 \mathrm{E}-01$

9.96650E-0 1

9. $96540 \mathrm{E}-01$

$96441 \mathrm{E}-01$

9. $96354 \mathrm{E}-01$

9.96281E-01

$9.96215 E-01$

$9.96157 \mathrm{E}-01$ 


\section{IAPT}

TIME

1. $00000 \mathrm{E}+00$

2. $00000 \mathrm{E}+00$

3. $00000 \mathrm{E}+00$
3.

$3.00000 \mathrm{E}+00$
4. $00000 \mathrm{E}+00$

5. $00000 \mathrm{E}+00$

6. $00000 E+00$

7. $00000 \mathrm{E}+00$

8. $00000 \mathrm{E}+00$

$9.00000 \mathrm{E}+00$

1. $00000 \mathrm{E}+01$

1. $10000 E+01$

1. $20000 \mathrm{P}_{1}+01$

1. $30000 \mathrm{E}+01$

$1.40000 E+01$

$1.60000 \mathrm{E}+01$

$1.60000 \mathrm{E}+01$
$1.70000 \mathrm{E}+01$

$1.90000 E+01$

$1.90000 \mathrm{E}+01$

$2.00000 \mathrm{E}+01$

2. 1000OE + 01

$2.20000 \mathrm{E}+01$

2. $30000 \mathrm{E}+01$

$2.30000 \mathrm{E}+01$
$2.40000 \mathrm{E}+01$

$2.50000 \mathrm{E}+01$

$2.60000 \dot{\mathrm{E}}+01$

2.70000E+01

$2.80000 E+01$

2. $90000 \mathrm{E}+01$

3. $00000 \mathrm{E}+01$

$3.10000 E+01$

3. $20000 E+01$

3. $30000 E+01$

$3.40000 \mathrm{E}+01$

$3.50000 \mathrm{E}+01$

$3.60000 \mathrm{E}+01$

$3.70000 E+01$

$3.80000 E+01$

3. $90000 \mathrm{E}+01$

4. $00000 \mathrm{E}+01$

4. $10000 \mathrm{E}+01$

4. $20000 \mathrm{E}+01$

$4.30000 E+01$

$4.40000 E+01$

4. $50000 \mathrm{E}+01$

$4.60000 E+01$

$4.70000 \mathrm{E}+01$

$4.70000 E+01$

$4.80000 E+01$

4. $90000 \mathrm{E}+01$
$5.00000 \mathrm{E}+01$

TR 1

TP 1

7. $30000 \mathrm{E}+02$

7. $30000 \mathrm{E}+02$

7. $30000 \mathrm{E}+02$

7. $30000 \mathrm{E}+02$

7. $30000 \mathrm{E}+02$

7. $30000 E+02$

7. $30000 E+02$

$30004 E+02$

$.30426 E+02$

$.30909 E+02$

7. $31027 \mathrm{E}+02$

7. $30931 \mathrm{E}+02$

7. $30788 \mathrm{E}+02$

7. $30661 \mathrm{E}+02$

7. $30554 \mathrm{E}+02$

7. $30458 \mathrm{E}+02$

$7.30363 \mathrm{E}+02$

7. $30262 E+02$

$7.30153 \mathrm{E}+02$

7. $30041 E+02$

7. $29954 \mathrm{E}+02$

$7.29897 \mathrm{E}+02$

$7.29859 \mathrm{E}+02$

$7.29829 \mathrm{E}+02$

7. $29802 \mathrm{E}+02$

$7.29778 E+02$

$7.29757 \mathrm{E}+02$

7. $29740 \mathrm{E}+02$

7. $29728 \mathrm{E}+02$

7. $29730 \mathrm{E}+02$

7. $29772 \mathrm{E}+02$

7. $29858 \mathrm{E}+02$

7. $29979 E+02$

7. $30123 \mathrm{E}+02$

7. $30281 \mathrm{E}+02$

$7.30442 \mathrm{E}+02$

7. $30598 \mathrm{E}+02$

7. $30740 \mathrm{E}+02$

7. $30865 \mathrm{E}+02$

7. $30970 \mathrm{E}+02$

7. $31055 \mathrm{E}+02$

$7.31120 \mathrm{E}+02$

7. $31166 \mathrm{E}+02$

7. $31195 E+02$

7. $31210 \mathrm{E}+02$

7. $31212 \mathrm{E}+02$

7. $31203 \mathrm{E}+02$

7. $31184 \mathrm{E}+02$

7. $31155 \mathrm{E}+02$

7. $31118 \mathrm{E}+02$

7. $31071 \mathrm{E}+02$
7. $30000 \mathrm{E}+02$

7. $30000 \mathrm{E}+02$

7. $30000 \mathrm{E}+02$

7. $30000 \mathrm{E}+02$

7. $30000 \mathrm{E}+02$

7. $30000 \mathrm{E}+02$

7. $30000 \mathrm{E}+02$

7. $30000 \mathrm{E}+02$

7. $30012 \mathrm{E}+02$

7. $30060 \mathrm{E}+02$

7. $30125 \mathrm{E}+02$

7. $30185 E+02$

7. $30232 \mathrm{E}+02$

7. $30266 \mathrm{E}+02$

7. $30289 E+02$

7. $30304 \mathrm{E}+02$

7. $30312 \mathrm{E}+02$

7. $30312 \mathrm{E}+02$

7. $30304 \mathrm{E}+02$

7. $30290 \mathrm{E}+02$

7. $30270 \mathrm{E}+02$

$7.302 .46 E+02$

7. $30220 \mathrm{E}+02$

7. $30194 \mathrm{E}+02$

7. $30168 \mathrm{~F}+02$

7. $30142 \mathrm{E}+02$

7. $30116 \mathrm{E}+02$

7. $300918+02$

7. $30066 \mathrm{E}+02$

$7.30043 \mathrm{E}+02$

7. $30022 \mathrm{E}+02$

$7.30008 E+02$

7. $30002 \mathrm{E}+02$

$7.30005 B+02$

7. $30019 E+02$

7. $30042 E+02$

7. $30075 E+02$

7. $30116 \mathrm{E}+02$

7. $30163 \mathrm{E}+02$

7. $30215 E+02$

7. $30383 \mathrm{E}+02$

7. $30437 E+02$

7. $30490 \mathrm{E}+02$

7. $30539 E+02$

7. $30585 E+02$

7. $30627 \mathrm{E}+02$

7. $30696 \mathrm{E}+02$
7. $30724 \mathrm{E}+02$
$8.82865 \mathrm{E}+0$

8. $85107 \mathrm{E}+02$

$8.85610 \mathrm{E}+02$

$8.86172 \mathrm{E}+02$

$8.86251 E+02$

$8.85977 E+02$

$8.85548 E+02$

$8.85055 E+02$

$8.84548 E+02$

$8.84068 E+02$

$83626 \mathrm{E}+02$

8. $83198 E+02$

$8.82838 E+02$

$\theta .82593 E+02$

$8.82434 \mathrm{E}+02$

$8.82347 \mathrm{E}+02$

8. $82326 \mathrm{E}+02$

$8.82299 \mathrm{E}+02$

8. $82298 \mathrm{E}+02$

$8.82317 \mathrm{E}+02$

$8.82304 \mathrm{E}+02$

$8.82282 \mathrm{E}+02$

$8.82307 E+02$

8. $82348 \mathrm{E}+02$

$8.82319 \mathrm{E}+02$

$8.82320 \mathrm{E}+02$

$8.82327 E+02$

$8.82327 \mathrm{E}+02$

$8.82341 \mathrm{E}+02$

$8.82340 \mathrm{E}+02$

8. $82348 E+02$

$8.82365 \mathrm{E}+02$

$8.82378 \mathrm{E}+02$

$8.82413 E+02$

8. $82459 E+02$

8. $25252+02$

$8.82599 \mathrm{E}+02$

$8.82691 \mathrm{E}+02$

$8.82792 \mathrm{E}+02$

$8.82905 E+02$

$8.83150 E+02$

$8.83279 \mathrm{E}+02$

$8.83410 E+02$

$8.83541 \mathrm{E}+02$

$8.83670 \mathrm{E}+02$

E. $83797 \mathrm{E}+02$

$8.84042 \mathrm{E}+02$

$8.84105 \mathrm{E}+02$

8. $84267 \mathrm{E}+\mathrm{D} 2$
TF3

$1.03573 \mathrm{E}+03$

. $04021 \mathrm{E}+03$

$1.04122 E+03$

$1.04234 E+03$

$1.04250 \mathrm{E}+03$

$1.04195 E+03$

$1.04110 \mathrm{E}+03$

$1.04011 \mathrm{E}+03$

$1.03808 \mathrm{E}+03$

$1.03713 E+03$

$1.03621 \mathrm{E}+03$

$1.03544 E+03$

$1.03492 E+03$

$1.03439 \mathrm{E}+03$

列

$1.03429 \mathrm{E}+03$

$1.03429 E+03$

$1.03434 E+03$

$1.03434 \mathrm{E}+03$

$1.03432 E+03$

$1.03439 E+03$

$1.03450 E+03$

$1.03447 \mathrm{E}+03$

$1.03450 E+03$

$.03454 \mathrm{E}+03$

$456 E+03$

$1.03462 \mathrm{E}+03$

$1.03464 \mathrm{E}+03$

$1.03467 \mathrm{E}+03$

$1.03472 E+03$

$1.03482 \mathrm{E}+03$

$1.034908+03$

$1.03500 \mathrm{E}+03$

$1.03512 \mathrm{E}+03$

$1.03527 \mathrm{E}+03$

$1.03542 \mathrm{E}+03$

$1.03559 \mathrm{E}+03$

$1.03579 E+03$

$1.03518 \mathrm{~B}+03$

$1.03638 E+03$

$1.03659 \mathrm{E}+03$

$1.03680 E+03$

$1.03701 \mathrm{E}+03$

$.03722 E+03$

$1.03742 E+03$

$1.03751 \mathrm{E}+03$

1. $03781 \mathrm{E}+03$

TR 3

$9.94730 \mathrm{E}+02$

$9.95686 E+02$

$9.96298 \mathrm{E}+02$

$9.96428 E+02$

$9.96504 \mathrm{E}+02$

$9.96534 \mathrm{E}+02$

$9.96518 E+02$

$96459 E+02$

$96361 \mathrm{E}+0$

$9.96361 E+02$

$9.96063 E+02$

$9.95895 \mathrm{E}+02$

$9.95754 E+02$

$9.95631 \mathrm{E}+02$

$95520 E+02$

$9.95419 E+02$

$9.95326 E+02$

$9.95240 \mathrm{E}+02$

$9.95161 \mathrm{E}+02$

$.95086 E+02$

$9.95017 \mathrm{E}+02$

$9.94953 \mathrm{E}+02$

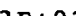

(1)

$4785 E+02$

$94650 \mathrm{~B}+02$ 
0. T I ME

1. $00000 \mathrm{E}+00$

2. $00000 E+00$

3. $00000 E+00$

4. $00000 \mathrm{E}+00$

$5.00000 \mathrm{E}+00$

$6.00000 \mathrm{E}+00$

$7.00000 \mathrm{E}+00$

$8.00000 \mathrm{E}+00$

$9.00000 E+00$

$1.00000 \mathrm{E}+0$

$1.10000 E+01$

$1.20000 \mathrm{E}+01$

1. $30000 \mathrm{E}+01$

$1.40000 E+01$

$1.50000 E+01$

$1.60000 \mathrm{E}+01$

$1.70000 \mathrm{E}+01$

$1.80000 E+01$

1. $90000 \mathrm{E}+01$

$2.00000 \mathrm{E}+01$

2. 10000E+01

$2.20000 E+01$

$2.30000 E+01$

$2.40000 E+01$

$2.50000 \mathrm{E}+01$

$2.60000 \mathrm{E}+01$

$2.70000 E+01$

$2.80000 \mathrm{E}+01$

$2.90000 \mathrm{E}+0$

3.00000E+01

3. $10000 E+01$

3. $20000 E+0$

$3.30000 \mathrm{E}+01$

$3.40000 \mathrm{E}+01$

$3.50000 \mathrm{E}+0$

$3.60000 \mathrm{E}+01$

$3.70000 \mathrm{E}+01$

$3.80000 \mathrm{E}+01$

$3.90000 \mathrm{E}+01$

4. $.00000 \mathrm{E}+01$

4. $10000 \mathrm{E}+01$

$4.20000 \mathrm{E}+01$

$4.300008+01$

$4.40000 \mathrm{~B}+01$

$4.50000 \mathrm{E}+01$

4. $60000 \mathrm{E}+0$

4. 70000E+01

$4.80000 \mathrm{E}+01$

4. $90000 E+01$

$5.00000 \mathrm{E}+0$
ENPP

1. $17000 \mathrm{E}+03$

1. $20261 \mathrm{E}+03$

1. $20394 E+03$

1. $20048 \mathrm{E}+03$

1. $19769 \mathrm{E}+03$

1. $19546 \mathrm{E}+03$

1. $19346 E+03$

1. $19145 E+03$

1. $18928 \mathrm{E}+03$

1. $18680 \mathrm{E}+03$

1. $18396 \mathrm{E}+03$

1. $18080 E+03$

1. $17831 \mathrm{R}+03$

1. $17712 \mathrm{E}+03$

1. $17639 \mathrm{R}+03$

1. $17581 \mathrm{E}+03$

1. $17526 \mathrm{E}+03$

1. $17473 E+03$

1. $17420 \mathrm{E}+03$

1. $17368 \mathrm{E}+03$

1. $17318 \mathrm{E}+03$

1. $17272 E+03$

1. $17228 \mathrm{E}+03$

1. $17186 \mathrm{E}+03$

1. $17146 \mathrm{E}+03$

1. $17108 \mathrm{E}+03$

1. $17071 E+03$

1. $17036 E+03$

1. $17003 \mathrm{~B}+03$

1. $16973 \mathrm{E}+03$

1. $16945 E+03$

$1.16918 E+03$

1. $16892 \mathrm{E}+03$

1. $16866 E+03$

1. $16837 \mathrm{E}+03$

1. $16806 \mathrm{~F}+03$

1. $16770 E+03$

1. $16733 \mathrm{E}+03$

$1.16690 \mathrm{E}+03$

1. $16643 \mathrm{E}+03$

1. $16593 E+03$

1. $16542 \mathrm{E}+03$

1. $16490 \mathrm{E}+03$

1. $16436 \mathrm{E}+03$

1. $16381 \mathrm{E}+03$

1. $16325 \mathrm{~F}+03$

1. $16268 \mathrm{E}+03$

1. $16212 E+03$

1. $16156 \mathrm{E}+03$

1. $16101 \mathrm{E}+03$

$1.16046 \mathrm{E}+03$
ETAPP

7. $92719 \mathrm{E}-01$

$7.93034 \mathrm{E}-01$

$7.92752 \mathrm{E}-01$

$7.92753 \mathrm{E}-01$

$7.92776 \mathrm{E}-01$

7. $92783 \mathrm{E}-01$

$7.92785 \mathrm{E}-01$

$7.92783 E-01$

. $92783 E-01$

$7.92777 \mathrm{E}-01$
$7.92768 \mathrm{E}-01$

7. $92756 \mathrm{E}-01$

$7.92741 \mathrm{E}-01$

$7.92744 \mathrm{E}-01$

$7.92749 \mathrm{E}-01$

7. $92749 \mathrm{E}-01$

$7.92747 \mathrm{E}-01$

$7.92744 \mathrm{E}-01$

7. $92741 \mathrm{E}-01$

$7.92738 \mathrm{E}-01$

$7.92735 \mathrm{E}-01$

$7.92732 \mathrm{E}-01$

7. $92727 \mathrm{E}-01$

7. $92724 \mathrm{E}-01$

$7.92722 \mathrm{E}-01$

7. $92719 \mathrm{E}-01$

$7.92717 E-01$

7. $92713 \mathrm{E}-01$

$7.92712 \mathrm{E}-01$

$7.92712 \mathrm{E}-01$

$7.92712 \mathrm{E}-01$

$7.92712 \mathrm{E}-01$

$7.92711 \mathrm{E}-01$

7. $92710 \mathrm{E}-01$

7. $92709 \mathrm{E}-01$

7. $92709 \mathrm{E}-01$

7. $92708 \mathrm{E}-01$

$7.92707 \mathrm{E}-01$

$7.92707 \mathrm{E}-01$

$7.92707 \mathrm{E}-01$

7. $92707 \mathrm{E}-01$

7. $92708 \mathrm{E}-01$

7. $92709 \mathrm{E}-01$

7. $92710 \mathrm{E}-01$

$7.92712 \mathrm{E}-01$

7. $92713 \mathrm{E}-01$

7. $92714 \mathrm{E}-01$
HOPP

$.00600 \mathrm{E}+03$

$1.03387 \mathrm{E}+03$

$.03583 \mathrm{E}+03$

$1.03084 \mathrm{E}+03$

$1.02630 \mathrm{E}+03$

$1.02403 E+03$

$1.02281 \mathrm{E}+03$

$1.02174 E+0$

$1.02047 \mathrm{E}+03$

$1.01890 E+03$

$1.01706 \mathrm{E}+03$

1. $01497 E+03$

$1.01345 E+03$

$1.01297 \mathrm{E}+03$

$1.01276 \mathrm{E}+03$

$1.01253 \mathrm{E}+03$

$1.01221 \mathrm{E}+03$

$1.01184 E+03$

$1.01143 E+03$

$1.01101 \mathrm{E}+03$

$1.01058 \mathrm{E}+03$

1. $01016 \mathrm{E}+03$

$1.00974 \mathrm{E}+03$

$1.00933 E+03$

$1.00893 E+03$

$1.00853 E+03$

$1.00814 \mathrm{E}+03$

$1.00775 \mathrm{E}+03$

$1.007378+03$

1. $00700 \mathrm{E}+03$

$1.00664 E+03$

$1.00628 \mathrm{E}+03$

$1.00593 E+03$

$1.00555 \mathrm{~B}+0$

$1.00511 \mathrm{E}+03$

$1.00465 \mathrm{E}+03$

1. $00417 \mathrm{E}+03$

$1.00374 \mathrm{E}+03$

$1.00330 E+03$

$1.00289 E+03$

$1.00250 \mathrm{E}+03$

$1.00214 \mathrm{E}+03$

$1.00182 E+03$

$1.00152 \mathrm{E}+03$

$1.00124 E+03$

$1.00097 \mathrm{E}+03$

$1.00072 \mathrm{E}+03$

$1.00050 \mathrm{E}+03$

1. $00029 \mathrm{E}+03$

$1.00010 \mathrm{E}+03$

9. $99922 E+02$
ETAIP

$02666 E-01$

$3.31999 \mathrm{E}+02$

$3.53354 \mathrm{E}+02$

$8.02047 \mathrm{E}-01 \quad 3.48577 \mathrm{E}+02$

$3.46460 \mathrm{E}+02$

$3.45508 E+02$

$8.02258 \mathrm{E}-01$

$8.02288 \mathrm{E}-01 \quad 3.43505 \mathrm{E}+02$

$8.02304 \mathrm{E}-01 \quad 3.42081 \mathrm{E}+02$

$8.02319 \mathrm{E}-01 \quad 3.40420 \mathrm{E}+02$

8.02337E-01 3.38582E+02

3. $37405 \mathrm{E}+02$

$8.02475 E-01 \quad 3.37232 E+02$

$8.02520 \mathrm{E}-013.37193 \mathrm{E}+02$

$8.02540 \mathrm{E}-01 \quad 3.37057 \mathrm{E}+02$

$8.02547 \mathrm{E}-01 \quad 3.36802 \mathrm{E}+02$

8.02551E-01 3. $36496 \mathrm{E}+02$

$8.02554 \mathrm{E}-01 \quad 3.36153 \mathrm{E}+02$

$8.02558 \mathrm{E}-01 \quad 3.35801 \mathrm{E}+02$

$8.02564 \mathrm{E}-01$ 3. $35454 \mathrm{E}+02$

$8.02570 \mathrm{E}-01 \quad 3.35116 \mathrm{E}+02$

$8.02576 \mathrm{E}-01 \quad 3.34785 \mathrm{~F}+02$

$8.02583 \mathrm{E}-01 \quad 3.34461 \mathrm{E}+02$

$8.02589 \mathrm{E}-01 \quad 3.34143 \mathrm{E}+02$

$8.02595 E-01 \quad 3.33830 E+02$

$8.02601 E-01 \quad 3.33523 E+02$

$0.02607 \mathrm{E}-01 \quad 3.33223 \mathrm{~B}+02$

B. $02612 \mathrm{E}-01 \quad 3.32930 \mathrm{E}+02$

$8.02618 \mathrm{E}-01 \quad 3.32643 \mathrm{E}+02$

$8.02623 \mathrm{E}-013.32362 \mathrm{E}+02$

$8.02628 E-01 \quad 3.32086 E+02$

$8.02633 E-01 \quad 3.31855 E+02$

B. $02630 \mathrm{E}-01 \quad 3.31699 \mathrm{E}+02$

$8.02622 \mathrm{E}-013.31497 \mathrm{~V}+02$

$8.02612 \mathrm{E}-013.31286 \mathrm{E}+02$

8.02602E-01 3.31061E+02

$8.02594 \mathrm{E}-013.30866 \mathrm{E}+02$

$8.02586 E-01 \quad 3.30664 E+02$

$8.02579 E-01 \quad 3.30474 E+02$

9.02572F-01 3. $30294 \mathrm{E}+02$

$8.02566 \mathrm{E}-01 \quad 3.30132 \mathrm{E}+02$

$8.02562 \mathrm{E}-01 \quad 3.29984 \mathrm{E}+02$

$8.02558 \mathrm{E}-013.29848 \mathrm{E}+02$

$8.02554 E-01 \quad 3.29719 E+02$

$8.02546 \mathrm{E}-01 \quad 3.29481 \mathrm{E}+02$

$8.02543 \mathrm{~F}-01 \quad 3.29376 \mathrm{E}+02$

$8.02540 \mathrm{E}-01 \quad 3.29281 \mathrm{E}+02$

$8.02538 \mathrm{E}-01 \quad 3.29192 \mathrm{~F}+02$

$8.02536 E-U 1 \quad 3.29113 E+02$

$4.44854 \mathrm{E}+02$ 
0 .

$1.00000 E+00$

$2.00000 E+00$

3. $00000 \mathrm{E}+00$

4. $00000 \mathrm{E}+00$

$5.00000 E+00$

$6.00000 \mathrm{E}+00$

7. $00000 \mathrm{E}+00$

8. $00000 \mathrm{E}+00$

$9.00000 \mathrm{E}+00$

$1.00000 \mathrm{E}+01$

$1.10000 \mathrm{E}+01$

1. $20000 \mathrm{E}+01$

$1.30000 E+01$

1. $40000 E+01$

$1.50000 B+01$

$1.60000 \mathrm{E}+01$

$1.70000 \mathrm{E}+01$

$1.80000 E+01$

$1.90000 E+01$

$2.00000 E+01$

$2.10000 \mathrm{E}+01$

$2.20000 \mathrm{E}+01$

$2.30000 E+01$

$2.40000 E+01$

$2.50000 E+01$

2. $60000 E+01$

$2.70000 \mathrm{E}+01$

$2.80000 \mathrm{E}+01$

$2.90000 E+01$

$3.00000 E+01$

$3.10000 E+01$

$3.20000 \mathrm{E}+01$

$3.30000 \mathrm{E}+01$

$3.40000 \mathrm{E}+01$

$3.50000 \mathrm{E}+01$

$3.60000 E+01$

$3.70000 E+01$

3. $80000 \mathrm{E}+01$

$3.90000 \mathrm{E}+01$

4. $00000 \mathrm{E}+01$

4. 10000E+01

4. $20000 \mathrm{E}+01$

4. $30000 \mathrm{E}+01$

4. $40000 E+01$

$4.500002+01$

$4.60000 \mathrm{E}+01$

$4.70000 \mathrm{E}+01$

4. $80000 E+01$

$4.90000 E+01$

$5.00000 \mathrm{E}+01$
TAUTS 9

3. $00987 \mathrm{E}+00$

$3.00975 E+00$

$3.00915 \mathrm{E}+00$

$3.00839 \mathrm{E}+00$

$3.00794 E+00$

$3.00817 \mathrm{E}+00$

$3.00919 \mathrm{E}+00$

$3.01084 \mathrm{E}+00$

$3.01280 \mathrm{E}+00$

$3.01479 \mathrm{E}+00$

$3.01669 \mathrm{E}+00$

$3.01845 E+00$

$3.02007 E+00$

$3.02154 \mathrm{E}+00$

$3.02281 \mathrm{E}+00$

$3.02385 \mathrm{E}+00$

$3.02464 \mathrm{E}+00$

$3.02521 \mathrm{E}+00$

$3.02559 \mathrm{E}+00$

$3.02584 \mathrm{E}+00$

$3.02596 E+00$

$3.02599 \mathrm{E}+00$

$3.02594 E+00$

$3.02581 \mathrm{~F}+00$

$3.02562 \mathrm{E}+00$

$3.02538 \mathrm{E}+00$

$3.02510 \mathrm{E}+Q 0$

$3.02479 \mathrm{E}+00$

$3.02445 E+00$

$3.02409 \mathrm{E}+00$

$3.02371 \mathrm{E}+00$

$3.02331 \mathrm{E}+00$

3. $02290 \mathrm{E}+00$

$3.02246 \mathrm{E}+00$

$3.02202 \mathrm{E}+00$

$3.02162 \mathrm{E}+00$

$3.02153 \mathrm{E}+00$

$3.02208 \mathrm{E}+00$

$3.02259 \mathrm{E}+00$

$3.02289 \mathrm{E}+00$

$3.02289 \mathrm{E}+100$

$3.02266 \mathrm{E}+00$

$3.02231 \mathrm{E}+00$

$3.02193 \mathrm{E}+00$

$3.02152 \mathrm{E}+00$

3. $02108 \mathrm{E}+00$

$3.02059 \mathrm{E}+00$

$3.02001 \mathrm{E}+00$

$3.01935 \mathrm{E}+00$

$.01865 E+00$

3. $01793 \mathrm{E}+00$
TAUHSA

. $55935 \mathrm{E}+00$

1. $55935 E+00$

1. $55934 \mathrm{E}+00$

1. $55935 \mathrm{E}+00$

1. $55953 \mathrm{E}+00$

1. $56002 \mathrm{E}+00$

1. $56085 E+00$

1. $56193 E+00$

$56307 \mathrm{E}+00$

1. $56416 \mathrm{E}+00$

1. $56513 \mathrm{E}+00$

1. $56599 \mathrm{E}+00$

1. $56673 \mathrm{E}+00$

1. $56737 \mathrm{E}+00$

1. $56789 E+00$

1. $56829 \mathrm{E}+00$

$1.56856 \mathrm{E}+00$

1. $56872 \mathrm{E}+00$

1. $56880 \mathrm{E}+00$

1. $56881 \mathrm{E}+00$

1. $56877 \mathrm{E}+00$

$1.55867 \mathrm{E}+00$

1. $56855 \mathrm{E}+00$

1. $56838 \mathrm{E}+00$

1. $56819 \mathrm{E}+00$

1. $56798 E+00$

1. $56775 \mathrm{E}+00$

1. $56750 \mathrm{E}+00$

1. $56725 \mathrm{E}+00$

1. $56698 \mathrm{E}+00$

1. $56672 E+00$

1. $56644 \mathrm{E}+00$

$1.56616 E+00$

$1.56587 \mathrm{E}+00$

1. $56557 \mathrm{E}+00$

1. $56528 E+00$

$1.56499 \mathrm{E}+00$

1. $56471 E+00$

1. $56444 \mathrm{E}+00$

1. $56418 \mathrm{E}+00$

1. $56392 \mathrm{E}+00$

1. $56365 E+00$

$1.56338 \mathrm{E}+00$

1. $56313 \mathrm{E}+00$

1. $56247 \mathrm{E}+00$

1. $562228+00$

1. $56194 \mathrm{E}+00$

$.56163 E+00$

1. $56129 E+00$
TAUH 4

$.50898 E+00$

.50816E+00

1. $50501 \mathrm{E}+00$

$1.50087 \mathrm{E}+00$

$1.49773 E+00$

$1.49676 \mathrm{E}+00$

$1.49796 \mathrm{E}+00$

$50034 \mathrm{E}+00$

1. $50258 \mathrm{~B}+00$

$.50443 \mathrm{E}+00$

1. $50608 \mathrm{E}+00$

1. $50778 \mathrm{E}+00$

$1.50962 E+00$

$51147 \mathrm{~F}+00$

.51312E

1. $51446 \mathrm{E}+00$

1. $51546 \mathrm{E}+00$

1. $51622 \mathrm{E}+00$

1. $51682 E+00$

$1.51777 \mathrm{E}$

$51811 \mathrm{E}+00$

1. $51836 \mathrm{E}+00$

1. $51853 \mathrm{E}+00$

$51863 E+00$

$51866 \mathrm{E}+00$

$.51858 \mathrm{E}+00$

$855 \mathrm{E}+00$

$51852 E+00$

1.51844E+00

1. $51831 \mathrm{E}+00$

. $51831 E+00$

$1.51788 \mathrm{E}+00$

1. $51759 \mathrm{E}+00$

$1.51728 E+00$

$51696 \mathrm{E}+00$

1.51666E+00

1. $51636 \mathrm{E}+00$

1. $51609 \mathrm{E}+00$

$1.51581 \mathrm{E}+00$

$1.51555 E+00$

. $51544 \mathrm{E}+00$

1. $1560 \mathrm{E}+00$

1. $51595 \mathrm{E}+00$

$1.51595 E+00$

(

$1.51621 \mathrm{E}+00$
TAUHS1

. $25263 \mathrm{E}+01$

2. $25286 \mathrm{E}+01$

2. $25413 \mathrm{E}+0$

$2.25568 \mathrm{E}+01$

2. $25637 \mathrm{E}+0$

2. $25577 \mathrm{E}+01$

2. $25427 \mathrm{E}+0$

2. $25141 \mathrm{E}+0$

. $25064 \mathrm{E}+01$

. $25000 \mathrm{E}+0$

2. $24936 E+01$

. $24868 \mathrm{E}+01$

2. $24805 E+01$

2. $24755 \mathrm{E}+01$

$2.24722 \mathrm{E}+01$

2. $24704 \mathrm{E}+0$

$2.24693 \mathrm{E}+01$

. $24686 \mathrm{E}+01$

$24680=01$

$2.24675 \mathrm{E}+0$

$2.24672 \mathrm{E}+01$

$2.24672 \mathrm{E}+0$

2. $24675 E+01$

2. $24679 \mathrm{E}+01$

$24684 E+01$

$2424690 E+01$

2. $24696 \mathrm{E}+01$

$2.24701 \mathrm{E}+0$

$2.24706 \mathrm{E}+0$

$.24719 E+01$

$2.24729 E+01$

$24743 E+01$

2. $24758 \mathrm{E}+0$

2. $24775 E+01$

. $24793 E+01$

$.24809 \mathrm{E}+01$

2. $24826 E+0$

$24841 E+01$

$24857 \mathrm{E}+0$

$2.24873 \mathrm{E}+0$

$24890 \mathrm{E}+0$

$2.24901 E+01$

$2.24907 \mathrm{E}+01$

$24913 \mathrm{E}+01$

$2.24928 \mathrm{E}+01$

2. $24953 E+0$

$.24983 \mathrm{E}+0$

$2.25015 E+01$

TAUTI7

$.80901 E+00$

$5.73098 \mathrm{E}+00$

$5.65995 E+C O$

$5.63729 E+00$

$5.64493 E+00$

$5.66182 \mathrm{E}+00$

$5.67587 \mathrm{E}+00$

$5.68656 \mathrm{E}+00$

$5.69614 \mathrm{E}+00$

$5.70634 \mathrm{E}+00$

$5.71796 \mathrm{E}+00$

$5.73117 E+00$

$5.74468 \mathrm{E}+00$

$5.75384 \mathrm{E}+00$

5. $75888 \mathrm{E}+00$

$5.76189 \mathrm{E}+00$

$5.76431 \mathrm{E}+00$

$5.76675 E+00$

$5.76938 \mathrm{E}+00$

$5.77220 \mathrm{E}+00$

5.77512E+

$5.77806 \mathrm{E}+00$

$5.78097 \mathrm{E}+00$

$5.78384 \mathrm{E}+00$

$5.78666 \mathrm{E}+00$ 
0.

1. $00000 \mathrm{E}+00$

2. $00000 \mathrm{E}+00$

3. $00000 \mathrm{E}+00$

4. $00000 \mathrm{E}+00$

$5.00000 \mathrm{E}+00$

$6.00000 \mathrm{~B}+00$

$7.00000 \mathrm{E}+00$

8. $00000 \mathrm{E}+00$

$9.00000 \mathrm{E}+00$

$1.00000 \mathrm{E}+01$

$1.10000 \mathrm{E}+01$

1. $20000 \mathrm{E}+01$

$1.30000 \mathrm{E}+01$

$1.40000 \mathrm{E}+01$

$1.50000 \mathrm{E}+01$

$1.60000 E+01$

$1.900000+01$

$1.90000 \mathrm{E}+01$

$2.00000 \mathrm{E}+01$

$2.10000 E+0$

$2.20000 \mathrm{E}+01$

$2.30000 \mathrm{E}+01$

$2.40000 \mathrm{E}+01$

$50000 \mathrm{E}+01$

$2.60000 \mathrm{E}+01$

$2.70000 E+01$

$2.80000 \mathrm{E}+01$

$2.90000 E+01$

$3.00000 E+01$

$3.10000 \mathrm{~F}+01$

3. $20000 \mathrm{E}+01$

$3.30000 \mathrm{E}+01$

$3.40000 E+01$

$3.50000 \mathrm{E}+01$

3. $60000 \mathrm{E}+01$

$3.70000 \mathrm{E}+01$

$3.80000 \mathrm{E}+0$

3. $90000 E+01$

4. $00000 \mathrm{E}+0$

4. 10000E+01

4. $20000 \mathrm{E}+01$

4. $30000 \mathrm{E}+0$

$4.40000 \mathrm{E}+01$

$4.50000 \mathrm{E}+01$

$4.60000 \mathrm{E}+01$

4. $70000 \mathrm{E}+01$

$4.80000 \mathrm{E}+01$

$4.90000 \mathrm{E}+01$

$5.00000 E+01$
TAUTI 1 2. $11632 E+01$ 2. $08791 \mathrm{E}+0$

$2.06206 \mathrm{~B}+01$ $2.05382 \mathrm{E}+01$

$2.05660 \mathrm{E}+01$

$2.06273 E+01$

$2.06780 E+01$

$2.07164 \mathrm{E}+01$

$2.07509 \mathrm{E}+01$

$2.07876 \mathrm{E}+01$

$2.08296 \mathrm{E}+01$

2. $08774 \mathrm{~B}+0$

$2.09262 E+01$

$2.09593 \mathrm{E}+01$

$2.09774 \mathrm{E}+01$

$2.09881 \mathrm{E}+01$

$2.09968 \mathrm{E}+01$

2. $10056 \mathrm{E}+0$

2. $10151 \mathrm{E}+01$

2. $10253 \mathrm{E}+01$

2. $10359 E+01$

2. $10466 \mathrm{E}+01$

2. $10572 E+01$

2. $10677 E+01$

2. $10780 \mathrm{E}+01$

2. $10882 \mathrm{E}+0$

2. $10982 E+01$

2. $11081 E+01$

2. $11177 \mathrm{E}+01$

2. $11272 \mathrm{E}+0$

2. $11365 E+01$

2. $11456 \mathrm{E}+01$

2. $11544 E+0$

2. $11617 E+01$

$2.11683 E+0$

$2.11749 E+01$

2. $11818 E+01$

2. $11886 \mathrm{E}+0$

2. $11952 E+O$

2. $12017 \mathrm{E}+0$

2. $12079 \mathrm{z}+01$

2. $12138 \mathrm{E}+01$

2. $12193 E+01$

2. $12244 \mathrm{E}+0$

2. $12290 E+01$

2. $12334 \mathrm{E}+0$

2. $12376 E+01$

2. $12451 \mathrm{E}+01$

2. $12484 \mathrm{E}+01$

2. $12515 \mathrm{E}+01$
TAUTP5

1.51904E+01 6.99342E+00

$97 \mathrm{E}+00$

$6.79904 \mathrm{E}+00$

1. $48127 \mathrm{E}+01 \quad 6.81857 \mathrm{E}+00$

$1.48528 \mathrm{E}+01 \quad 6.83717 \mathrm{E}+00$

1. $48823 E+01$

$6.86284 \mathrm{E}+00$

$1.49581 \mathrm{E}+01$ E. $88645 \mathrm{E}+00$

$1.49871 \mathrm{E}+01 \quad 6.89996 \mathrm{E}+00$

$1.50202 E+01 \quad 6.91535 E+00$

$1.50568 \mathrm{E}+01$

$1.50894 E+01 \quad 6.94751 E+00$

$1.51059 \mathrm{E}+01 \quad 6.95521 \mathrm{E}+00$

$1.51152 \mathrm{E}+01 \quad 6.95961 \mathrm{E}+00$

$1.51222 \mathrm{E}+01 \quad 6.96288 \mathrm{E}+00$

$1.51286 \mathrm{E}+01 \quad 6.96585 \mathrm{E}+00$

$6.96866 \mathrm{E}+00$

$1.51407 \mathrm{E}+01 \quad 6.97148 \mathrm{E}+00$

1. $51467 \mathrm{E}+01 \quad 6.97425 \mathrm{E}+00$

$1.51524 \mathrm{E}+01 \quad 6.97689 \mathrm{E}+00$

$1.51578 E+01 \quad 6.97937 E+00$

$1.51628 E+01 \quad 6.98169 E+00$

$6.98385 \mathrm{E}+00$

$1.51722 \mathrm{E}+01 \quad 6.98585 \mathrm{E}+00$

$1.51766 \mathrm{E}+01 \quad 6.98771 \mathrm{E}+00$

$1.51808 \mathrm{E}+016.98946 \mathrm{E}+00$

$1.51847 E+01 \quad 6.99109 E+00$

$1.51885 \mathrm{E}+01 \quad 6.99264 \mathrm{E}+00$

$6.99419 E+00$

$1.51959 \mathrm{E}+01 \quad 6.99565 \mathrm{E}+00$

$1.51993 \mathrm{E}+01 \quad 6.99708 \mathrm{E}+00$

$1.52027 \mathrm{E}+01 \quad 6.99851 \mathrm{E}+00$

1. $52061 \mathrm{E}+01 \quad 6.99994 \mathrm{E}+00$

$1.52097 \mathrm{E}+01 \quad 7.00149 \mathrm{E}+00$

$1.52135 \mathrm{E}+017.00319 \mathrm{E}+00$

$1.52181 \mathrm{E}+01 \quad 7.00523 \mathrm{E}+00$

$1.52227 \mathrm{E}+017.00732 \mathrm{E}+00$

$1.52283 \mathrm{E}+01 \quad 7.00984 \mathrm{E}+00$

1. $52342 \mathrm{E}+01 \quad 7.01256 \mathrm{E}+00$

$1.52406 \mathrm{E}+01 \quad 7.01551 \mathrm{E}+00$

$1.52472 \mathrm{E}+01 \quad 7.01858 \mathrm{E}+00$

$1.52541 E+01 \quad 7.02179 E+00$

$1.52613 \mathrm{E}+01 \quad 7.02509 \mathrm{E}+00$

1. $52685 \mathrm{E}+017.02850 \mathrm{E}+00$

$1.52761 \mathrm{E}+01 \quad 7.03202 \mathrm{E}+00$

$1.52837 \mathrm{E}+01 \quad 7.03562 \mathrm{E}+00$

$1.52914 \mathrm{E}+01 \quad 7.03922 \mathrm{E}+00$

$1.52990 \mathrm{E}+01 \quad 7.04282 \mathrm{E}+00$

$1.53067 \mathrm{E}+017.04644 \mathrm{E}+00$

$1.53142 \mathrm{E}+01 \quad 7.05003 \mathrm{E}+00$ 
MAX. TEMPS. IN THE AVG. AND HOT CHANNELS AND THEIR AXIAL LOCATIONS L. IS T, TPMAX, TPMM, YCHAX, T CMAX, YCMM, TCMM

TIM

1. $00000 \mathrm{E}+00$

$2.00000 E+00$

3. $00000 \mathrm{E}+00$

4. $00000 \mathrm{E}+00$

$5.00000 E+00$

$6.00000 \mathrm{E}+00$

$7.00000 E+00$

$8.00000 \mathrm{E}+00$

$9.00000 \mathrm{E}+00$

$1.00000 \mathrm{E}+01$

1. $10000 \mathrm{E}+01$

1. $20000 \mathrm{E}+01$

$1.30000 \mathrm{E}+01$

$1.40000 \mathrm{E}+01$

1. $50000 \mathrm{E}+01$

$1.60000 \mathrm{E}+01$

1. $70000 E+01$

1. $80000 E+01$

1. $90000 E+01$

2. $00000 \mathrm{E}+01$

2. $10000 E+01$

2. $20000 \mathrm{E}+01$

$2.30000 E+01$

$2.40000 E+01$

$2.50000 \mathrm{~F}+01$

$2.60000 \mathrm{E}+01$

$2.70000 E+01$

$2.80000 E+01$

$2.90000 \mathrm{E}+01$

3. $00000 E+01$

$3.10000 E+01$

3. $20000 \mathrm{E}+01$

3. $30000 \mathrm{E}+01$

$3.40000 \mathrm{E}+01$

$3.50000 E+01$

3. $60000 \mathrm{E}+01$

$3.70000 E+01$

$3.80000 \mathrm{E}+01$

3. $90000 \mathrm{E}+01$

4. $00000 \mathrm{E}+01$

$4.10000 E+01$

4. 20000E+01

4. $30000 E+01$

$4.40000 \mathrm{E}+01$

$4.50000 \mathrm{E}+01$

4. $60000 \mathrm{E}+01$

$4.70000 E+01$

$4.80000 \mathrm{E}+01$

4. $90000 E+01$

$5.00000 \mathrm{E}+01$
TPMAX

$.03573 \mathrm{E}+03$

$1.05222 \mathrm{E}+03$

$1.04413 \mathrm{E}+03$

$1.04164 \mathrm{E}+03$

$1.04028 \mathrm{E}+03$

$1.03911 \mathrm{E}+03$

$.03797 \mathrm{E}+0$

$1.03683 \mathrm{E}+03$

$1.03570 \mathrm{E}+03$

$1.03461 \mathrm{E}+03$

1. $03360 E+03$

$1.03262 \mathrm{E}+03$

$1.03310 \mathrm{E}+03$

$1.03348 \mathrm{E}+03$

$1.03371 \mathrm{E}+03$

$.03383 \mathrm{E}+03$

1. $03397 \mathrm{E}+03$

$1.03406 \mathrm{E}+03$

$.034138+03$

$1.03419 E+03$

$1.03424 \mathrm{E}+03$

$1.03429 \mathrm{E}+03$

$1.03434 \mathrm{E}+03$

1. $03438 \mathrm{E}+03$

$.03442 E+03$

$1.03446 \mathrm{E}+03$

1. $03450 \mathrm{E}+03$

$1.03454 E+03$

$1.03458 E+03$

$1.03462 E+03$

$.03465 \mathrm{E}+03$

$1.03469 \mathrm{E}+03$

$1.03476 \mathrm{E}+03$

$1.03485 \mathrm{E}+03$

$1.03498 \mathrm{E}+03$

$1.03507 \mathrm{E}+03$

1. $03519 E+03$

1. $03534 \mathrm{E}+03$

$1.03552 \mathrm{E}+03$

$1.03568 \mathrm{E}+03$

$1.03587 \mathrm{E}+03$

$1.03607 \mathrm{E}+03$

$.03629 E+03$

$1.03651 \mathrm{E}+03$

$1.03669 \mathrm{E}+03$

$1.03690 \mathrm{E}+03$

$1.03713 \mathrm{E}+03$

$.03733 \mathrm{E}+03$

$.03752 \mathrm{E}+03$

$1.03772 E+03$

1. $03790 \mathrm{E}+03$
TP M

. $33303 E+03$

1. $35580 \mathrm{E}+03$

1. $34463 \mathrm{E}+03$

1. $34120 \mathrm{E}+03$

$1.33932 \mathrm{E}+03$

1. $33770 \mathrm{E}+03$

1. $33613 E+03$

1. $33455 E+03$

1. $33299 \mathrm{E}+03$

1. $33149 \mathrm{E}+03$

1. $33007 E+03$

1. $32872 \mathrm{E}+03$

1. $32937 \mathrm{E}+03$

1. $32990 \mathrm{E}+03$

1. $33022 E+03$

1. $33037 \mathrm{E}+03$

1. $33057 \mathrm{E}+03$

1. $33069 \mathrm{E}+03$

. $33078 E+0$

1. $33087 \mathrm{E}+03$

$1.33095 E+03$

1. $33102 E+03$

$1.33108 E+03$

1. $33115 E+03$

1. $33121 \mathrm{E}+03$

1. $33127 \mathrm{E}+03$

1. $33133 E+03$

1. $33138 \mathrm{E}+03$

$1.33144 \mathrm{E}+03$

1. $33149 E+03$

$33155 \mathrm{E}+0$

1. $33160 \mathrm{E}+03$

1. $33170 \mathrm{E}+03$

1. $33182 \mathrm{E}+03$

1. $33200 \mathrm{E}+03$

1. $33212 \mathrm{E}+03$

1. $33229 E+03$

1. $3324 \mathrm{gE}+03$

1. $33272 \mathrm{E}+03$

1. $33294 E+03$

$1.33320 \mathrm{E}+03$

1. $33347 E+03$

1. $33376 \mathrm{E}+03$

1. $33405 E+03$

1. $33430 \mathrm{E}+03$

1. $33458 \mathrm{E}+03$

1. $33489 \mathrm{E}+03$

1. $33515 \mathrm{E}+03$

1. $33542 \mathrm{E}+03$

1. $33569 E+03$

$1.33594 \mathrm{E}+03$
Y CMAX

$.50000 E+00$

$1.50000 \mathrm{E}+00$

$1.50000 \mathrm{E}+00$

$1.50000 \mathrm{E}+00$

$1.50000 E+00$

1. $50000 \mathrm{E}+00$

. $50000 \mathrm{E}+00$

1. $50000 \mathrm{E}+00$

$1.50000 \mathrm{E}+00$

1. $50000 E+00$

$1.50000 \mathrm{E}+00$

$1.50000 \mathrm{E}+00$

$50000 E+00$

$1.50000 \mathrm{E}+00$

1. $50000 \mathrm{E}+00$

1. $50000 \mathrm{E}+00$

$1.50000 \mathrm{E}+00$

$1.50000 \mathrm{E}+00$

$.50000 \mathrm{E}+00$

1. $50000 \mathrm{E}+00$

$1.50000 \mathrm{E}+00$

$1.50000 E+00$

$1.50000 \mathrm{E}+00$

$.50000 \mathrm{E}+00$

$50000 E+00$

$1.50000 \mathrm{E}+00$

$1.50000 E+00$

$1.50000 E+00$

1. $50000 \mathrm{E}+00$

$.50000 \mathrm{E}+00$

$50000 \mathrm{D}+00$

1. $50000 \mathrm{E}+00$

. $50000 \mathrm{E}+00$

$1.50000 \mathrm{E}+00$

1. $50000 E+00$

$1.50000 \mathrm{E}+00$

$.50000 E+00$

$1.50000 \mathrm{E}+00$

. $50000 \mathrm{E}+00$

1. $50000 \mathrm{E}+00$

$1.50000 \mathrm{E}+00$

$1.50000 E+00$

$1.50000 E+00$

1. $50000 \mathrm{E}+00$

. $50000 \mathrm{E}+00$

1. $50000 \mathrm{E}+00$

1. $50000 \mathrm{E}+00$

1. $50000 E+00$

$.50000 \mathrm{E}+00$

1. $50000 \mathrm{E}+00$
TCHAX

$4583 E+03$

$1.06303 E+03$

$.05470 \mathrm{E}+03$

$.05211 \mathrm{E}+03$

$1.05069 E+03$

$1.04947 \mathrm{E}+03$

$04828 E+03$

$1.04708 E+03$

$.04590 \mathrm{E}+03$

$1.04477 \mathrm{E}+03$

$1.04370 E+03$

$.04267 \mathrm{E}+03$

$04315 \mathrm{E}+03$

$1.043545+03$

$.04377 \mathrm{E}+03$

$1.04389 E+03$

$1.04403 E+03$

$.04412 E+03$

$.044198+03$

$04425 E+03$

$.04430 \mathrm{E}+03$

$1.04435 E+03$

$1.04440 E+03$

$.04444 \mathrm{E}+03$

$.04448 \mathrm{E}+03$

$1.04453 E+03$

$.04456 E+03$

$1.04460 \mathrm{E}+03$

$1.04464 E+03$

$1.04468 \mathrm{E}+03$

$.04472 E+03$

$1.04475 E+03$

$.04483 E+03$

$.04491 E+03$

$1.04505 E+03$

$.04514 \mathrm{E}+03$

$1.04526 \mathrm{E}+03$

$.04541 E+03$

$1.04559 \mathrm{E}+03$

$.04576 \mathrm{E}+03$

1. $04595 \mathrm{E}+03$

$1.04637 E+03$

$1.04659 \mathrm{E}+03$

1. $04678 \mathrm{E}+03$

$1.04699 E+03$

$.04722 E+03$

$1.04741 E+03$

$1.04761 \mathrm{E}+03$

$1.04781 \mathrm{E}+03$

$1.04800 \mathrm{E}+03$

YCUH

$1.50000 \mathrm{E}+00$

$1.50000 E+00$

1. $50000 E+00$

$1.50000 \mathrm{E}+00$

$1.50000 \mathrm{E}+00$

$1.50000 E+00$

1. $50000 E+00$

$1.50000 \mathrm{E}+00$

$1.50000 \mathrm{E}+00$

$1.50000 E+00$

$1.50000 \mathrm{E}+00$

$.50000 E+00$

$1.50000 E+00$

$1.50000 E+00$

$1.50000 \mathrm{E}+00$

$1.50000 \mathrm{E}+00$

$1.50000 \mathrm{E}+00$

$.50000 E+00$

$1.50000 \mathrm{E}+00$

1. $50000 \mathrm{E}+00$

$1.50000 \mathrm{E}+00$

$1.50000 \mathrm{E}+00$

$1.50000 \mathrm{E}+00$

$1.50000 E+00$

$1.50000 E+00$ 
0 .

$1.00000 E+00$ $2.00000 \mathrm{E}+00$ 3. $00000 \mathrm{E}+00$ 4. $00000 \mathrm{E}+00$ $5.00000 E+00$ $6.00000 E+00$ $7.00000 \mathrm{E}+00$ $8.00000 \mathrm{E}+00$ $9.00000 \mathrm{~F}+00$ 1. $00000 E+01$ 1. $10000 E+01$ 1. $20000 \mathrm{E}+01$ $1.30000 E+01$ $1.40000 E+01$ $1.50000 E+01$ $1.60000 \mathrm{E}+01$ $1.70000 \mathrm{E}+01$ $1.80000 \mathrm{E}+0$

1. $90000 \mathrm{E}+01$ $2.00000 E+01$ $2.10000 \mathrm{E}+01$ $2.20000 \mathrm{E}+01$ $2.30000 \mathrm{E}+01$ $2.40000 E+01$ $2.50000 \mathrm{E}+01$ $2.60000 \mathrm{E}+01$ $2.70000 E+01$ 2. $80000 \mathrm{E}+01$ $2.90000 \mathrm{E}+01$

3. $00000 E+01$

$3.10000 E+01$

$3.20000 E+01$ 3. $30000 \mathrm{E}+01$ $3.40000 E+01$ $3.50000 \mathrm{E}+01$ $3.60000 E+01$

3. $70000 \mathrm{E}+01$ $3.80000 \mathrm{E}+01$ $3.90000 E+01$ 4. $00000 E+01$ 4. 10000E+01 4. $20000 E+01$ 4. $30000 E+01$ $4.40000 \mathrm{E}+01$ 4. $50000 \mathrm{E}+01$ $4.60000 \mathrm{E}+01$ $4.70000 \mathrm{E}+01$ 4. $80000 \mathrm{E}+01$ 4. $90000 \mathrm{E}+01$ $5.00000 \mathrm{E}+01$

\section{TSMAX}

$1.67889 \mathrm{E}+03$

$1.73998 \mathrm{E}+03$ $1.72042 \mathrm{E}+03$ $1.71092 \mathrm{E}+03$ $1.70507 E+03$ $1.70051 \mathrm{E}+03$ $1.69632 E+03$ $1.69220 \mathrm{E}+03$ $1.68804 \mathrm{E}+03$ 1. $68381 \mathrm{E}+03$ $1.67954 \mathrm{E}+03$ $1.67525 \mathrm{E}+03$ 1. $675305+03$ $1.67576 E+03$ $1.67607 \mathrm{E}+03$ 1. $67612 \mathrm{E}+03$ $1.67626 \mathrm{E}+03$ $1.67624 \mathrm{E}+03$ 1. $67618 \mathrm{~B}+03$ $1.67612 \mathrm{E}+03$ 1. $67605 \mathrm{E}+03$ $1.67598 \mathrm{E}+03$ $1.67595 \mathrm{E}+03$ $1.67594 \mathrm{E}+03$ $1.67588 \mathrm{E}+03$ $1.67585 \mathrm{E}+03$ $1.67583 E+03$ $1.67581 \mathrm{E}+03$ $1.67579 \mathrm{E}+03$ $1.67578 \mathrm{~B}+03$ $1.67576 \mathrm{E}+03$ $1.67575 \mathrm{E}+03$ $1.67579 E+03$ $1.67588 \mathrm{E}+03$ $1.67605 E+03$ $1.67605 \mathrm{E}+03$ $1.67611 \mathrm{E}+03$ $1.67619 \mathrm{E}+03$ $1.67631 \mathrm{E}+03$ $1.67637 \mathrm{E}+03$ $1.67647 \mathrm{E}+03$ $1.67658 \mathrm{E}+03$ $1.67672 \mathrm{E}+03$ $1.67686 \mathrm{E}+03$ $1.67689 \mathrm{E}+03$ $1.67700 \mathrm{E}+03$ $1.67716 \mathrm{E}+03$ $1.67723 E+03$ $1.67733 E+03$ $1.67738 \mathrm{E}+03$ 1. $67751 \mathrm{E}+03$
YSHM 2. $95874 F-01$ . $92356 \mathrm{E}-01$ $2.90262 \mathrm{E}-01$ 2. $90866 \mathrm{E}-01$ 2. $91428 \mathrm{~B}-01$ 2.91753E-01 2. $91976 \mathrm{E}-01$ 2. $92173 \mathrm{E}-01$ $2.92388 \mathrm{E}-01$ 2. $92661 \mathrm{E}-01$ $2.93013 \mathrm{E}-01$ $2.93430 \mathrm{E}-01$ 2. $93937 \mathrm{E}-01$ $2.94191 \mathrm{E}-01$ $2.94333 \mathrm{E}-0$ $2.94440 \mathrm{E}-01$ 2. $94544 \mathrm{E}-01$ $2.94655 E-01$ $2.94765 \mathrm{E}-01$ 2. $94870 \mathrm{E}-01$ 2. $94979 \mathrm{k}-01$ 2. $95084 \mathrm{E}-0$ $2.95170 \mathrm{~B}-01$ $2.95247 \mathrm{E}-01$ 2. $95340 \mathrm{E}-01$ $2.95421 \mathrm{E}-0$ $2.95497 \mathrm{E}-01$ 2. $95571 \mathrm{E}-01$ $2.95637 \mathrm{E}-01$ 2. 95709R-0 2. $95775 \mathrm{E}-01$ 2. $95838 \mathrm{E}-01$ 2. $95908 \mathrm{E}-01$ $2.95978 \mathrm{E}-01$ 2. $96059 \mathrm{E}-0$ 2. $96142 \mathrm{E}-01$ 2. $96243 \mathrm{E}-01$ 2. $96349 \mathrm{E}-01$ 2. $96476 \mathrm{E}-0$ 2. $96609 \mathrm{E}-01$ 2. $96753 \mathrm{E}-0$ $2.96905 E-01$ 2. $97063 E-01$ 2. $97226 \mathrm{E}-0$ 2. $97388 \mathrm{E}-0$ 2. $97557 \mathrm{E}-01$ $2.97730 \mathrm{E}-0$ $2.97899 E-01$ 2. $98068 E-01$ $2.98251 \mathrm{E}-0$ 2. $98403 \mathrm{E}-01$

\section{TSK}

. $1288 \mathrm{E}+0$ $2.510 \mathrm{C} 8 \mathrm{E}+0$ $2.47946 E+0$ $2.46431 \mathrm{E}+03$ $2.45497 E+03$ $2.44770 E+03$ $2.44104 E+03$ $2.43449 E+03$ $2.42787 \mathrm{E}+0$ $2.42112 \mathrm{E}+0$ $2.41429 E+03$ $2.40742 \mathrm{E}+03$ $2.40741 \mathrm{E}+03$ $.40810 \mathrm{E}+0$ $2.40855 E+0$ $2.40861 \mathrm{E}+0$ $2.40882 \mathrm{E}+03$ $2.40877 \mathrm{E}+03$ $2.40866 \mathrm{E}+03$ $2.40856 \mathrm{E}+0$ $2.40843 E+03$ $2.40832 \mathrm{E}+03$ $2.40826 \mathrm{E}+03$ $2.40824 \mathrm{E}+03$ $2.40815 \mathrm{E}+03$ $2.40809 E+03$ $2.40806 E+03$ $2.40802 \mathrm{E}+03$ $2.408008+03$ $2.40797=+03$ $2.40794 E+03$ $2.40791 \mathrm{E}+03$ $2.40798 E+03$ $2.40809 \mathrm{E}+03$ $2.40835 E+03$ $2.40833 E+03$ $2.40839 E+03$ $2.40849 \mathrm{E}+03$ $2.40865 E+0$ $2.40871 \mathrm{E}+0$ $2.40882 \mathrm{E}+03$ $2.40895 E+03$ $2.40914 E+03$ $2.40932 E+03$ $2.40932 E+03$ $2.40945 E+03$ $2.40965 E+03$ $2.40974 \mathrm{E}+03$ $2.40985 E+03$ . $40990 \mathrm{E}+03$ $2.41007 \mathrm{E}+03$
Y FMAX

$16110 \mathrm{E}-0$

1. $12965 \mathrm{E}-01$

1. $11973 \mathrm{E}-0$

1. $12244 \mathrm{E}-01$

1. $12593 \mathrm{E}-01$

1. 12895E-0

1. 13177E- 01

$1.13462 \mathrm{E}-0$

$13762 \mathrm{E}-0$

1. 14091E-01

1. $14457 \mathrm{E}-01$

1. $14853 \mathrm{E}-01$

1. $15218 \mathrm{E}-01$

$15417 \mathrm{E}-0$

. $15533 \mathrm{E}-01$

1. 15613E-0 1

1. 15679E-01

$15737 E-01$

1. 1579 1E-0 1

$1.15843 \mathrm{E}-0$

. $15892 \mathrm{E}-01$

$1.15938 \mathrm{E}-01$

1. $15979 \mathrm{E}-01$

1. $16017 \mathrm{E}-0$

i. $16055 \mathrm{E}-01$

$16090 \mathrm{E}-01$

1. $16124 \mathrm{E}-01$

1. 16155E-01

1. $16185 \mathrm{E}-01$

1. 16215E-0

1. $16243 \mathrm{E}-0$

$16270 \mathrm{E}-01$

1. $16297 \mathrm{E}-01$

1. $16323 \mathrm{E}-01$

1. $16351 \mathrm{E}-01$

$1.16379 \mathrm{E}-01$

$1.16415 \mathrm{E}-01$

1. $16450 \mathrm{E}-01$

1. 16493E-01

1. $16538 \mathrm{E}-0$

1. $16588 \mathrm{E}-01$

1. $16640 \mathrm{E}-0$

1. 16694E-01

$16748 \mathrm{E}-01$

$.16805 E-01$

1. $16863 \mathrm{E}-0$

1. $16922 \mathrm{E}-01$

1. $16981 \mathrm{E}-0$

1. $17040 \mathrm{E}-01$

1. $17101 \mathrm{E}-0$

1. $17158 \mathrm{E}-01$

TF MAX

$3.25303 E+03$

$3.45859 \mathrm{E}+03$

$3.41215 E+03$

$3.38490 \mathrm{E}+03$

. $36559 \mathrm{E}+03$

$34908 \mathrm{E}+03$

3. $33330 \mathrm{E}+03$

$3.31753 \mathrm{E}+03$

$3.30151 E+03$

$3.28511 \mathrm{E}+03$

$.26844 \mathrm{E}+03$

$25163 \mathrm{E}+03$

$3.24776 \mathrm{E}+03$

3. $24666 \mathrm{E}+03$

$3.24604 \mathrm{E}+03$

3. $24526 \mathrm{E}+03$

$3.24504 \mathrm{E}+03$

$3.24458 E+03$

$3.24409 \mathrm{E}+03$

$3.24364 \mathrm{E}+03$

$3.24324 \mathrm{E}+03$

$3.24289 \mathrm{E}+03$

$3.24262 \mathrm{E}+03$

$3.24239 \mathrm{E}+03$ 

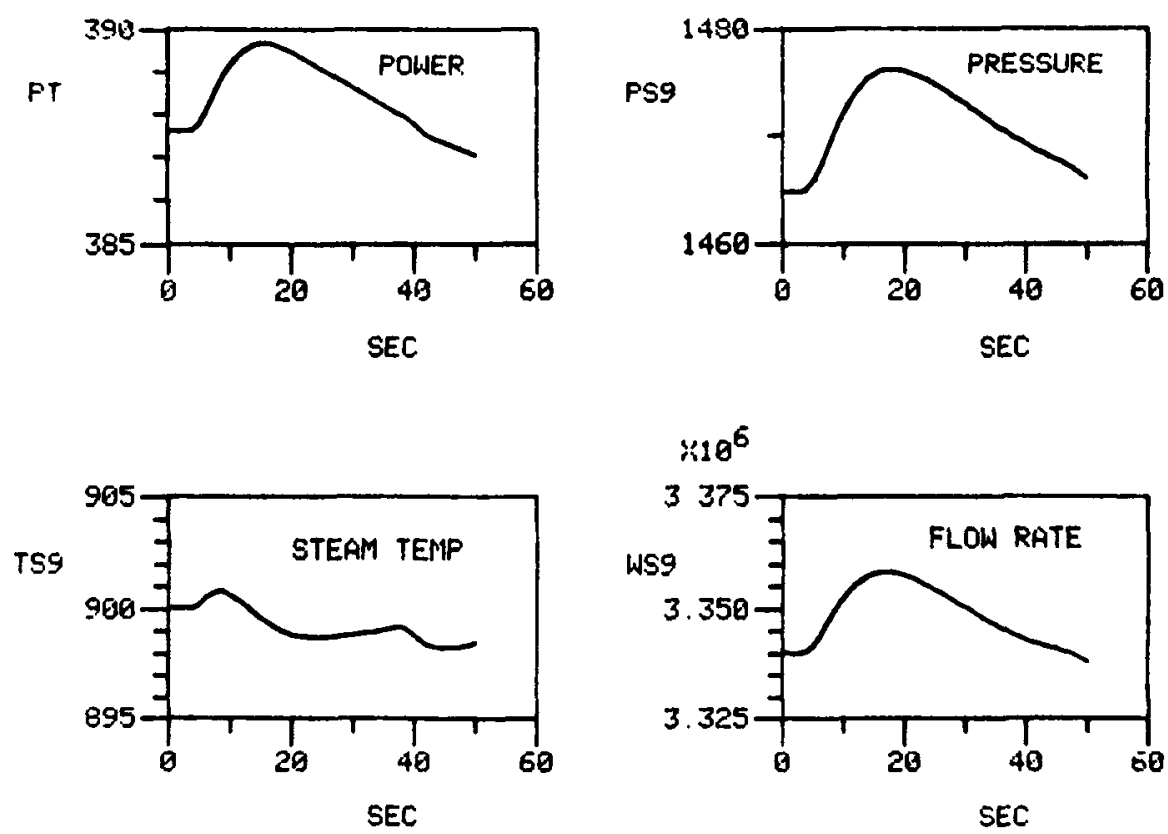

\section{SUPERHEATER STEAM}
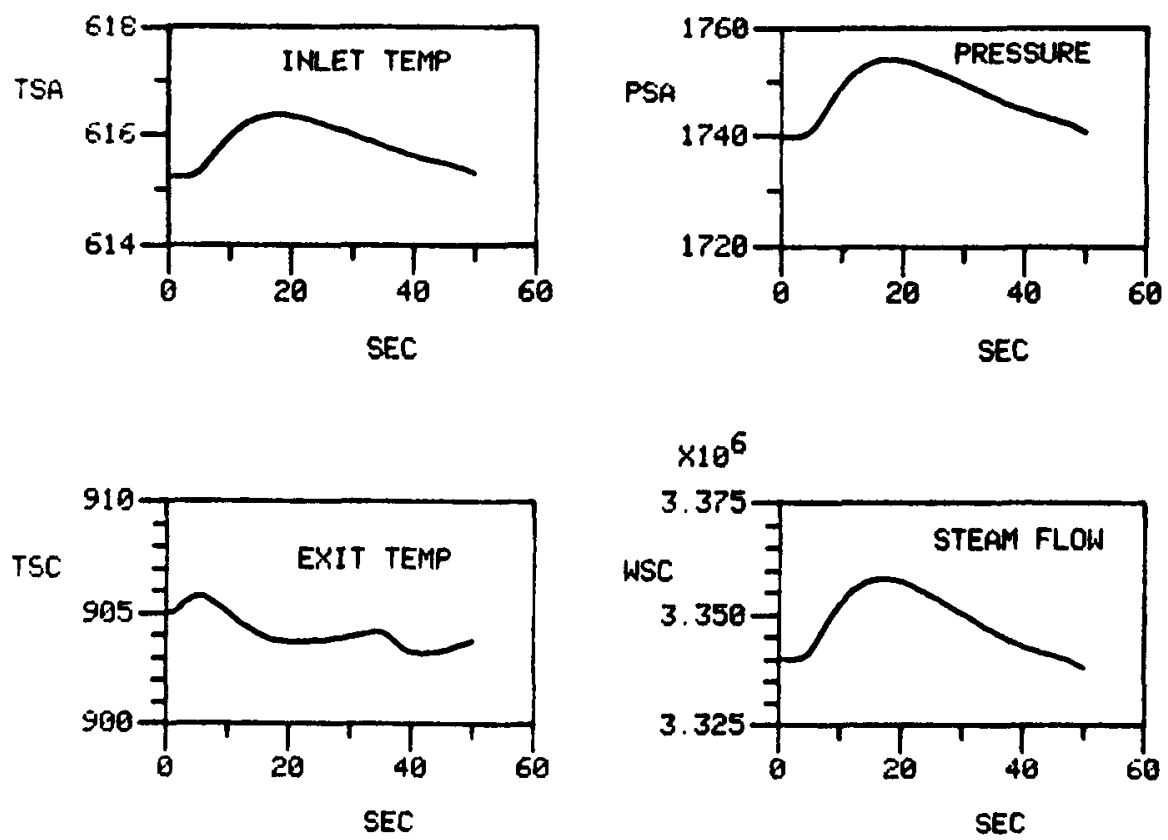

Fig. 2 Response of the CRBRP to a 10-cent Reactivity Step. 

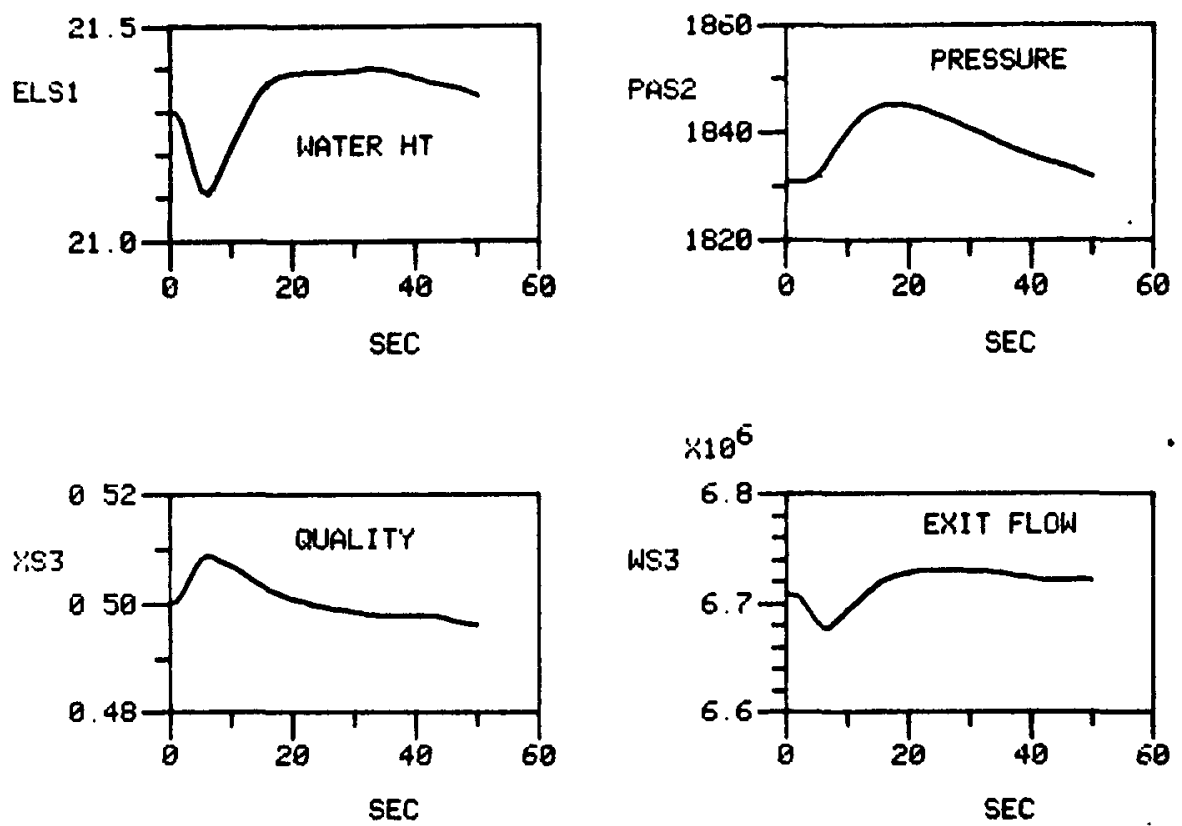

STEAM ORUM
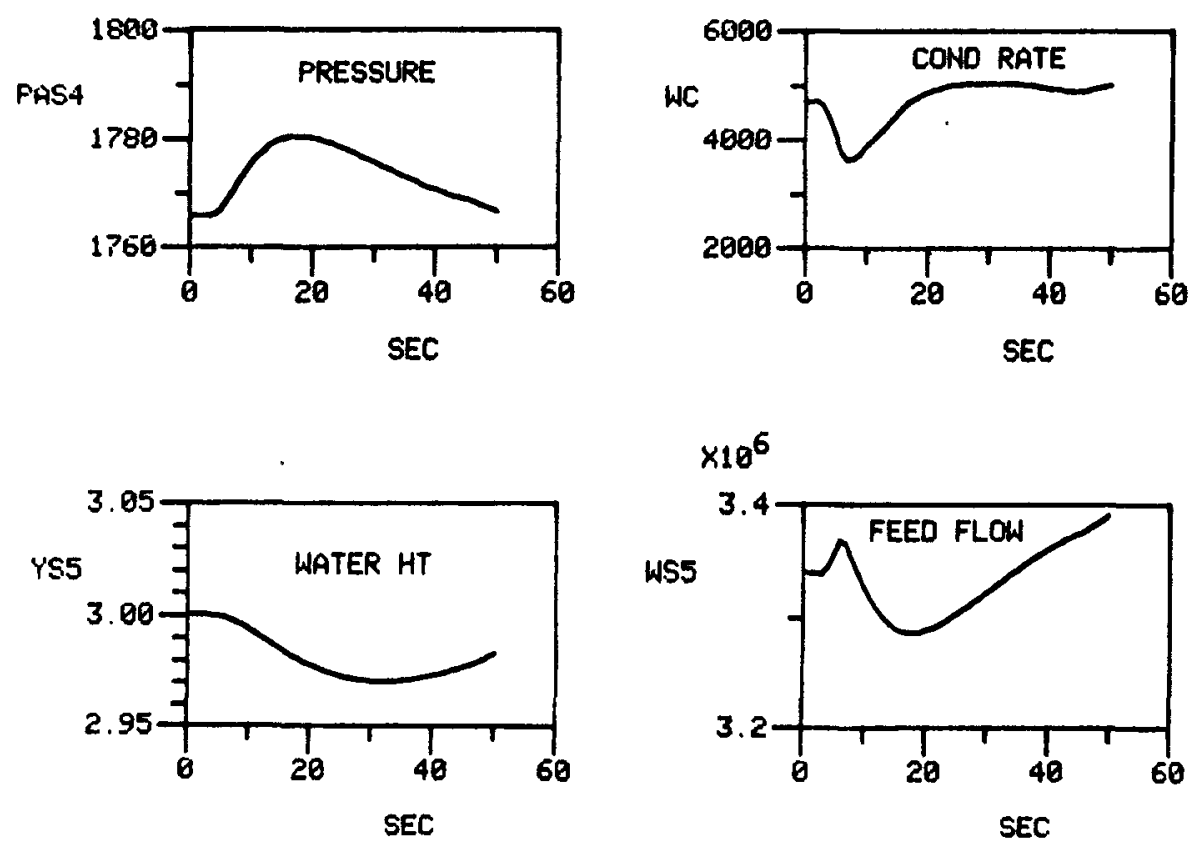

Fig. 3 Response of the CRBRP to a 10-cent Reactivity Step (Cont.). 

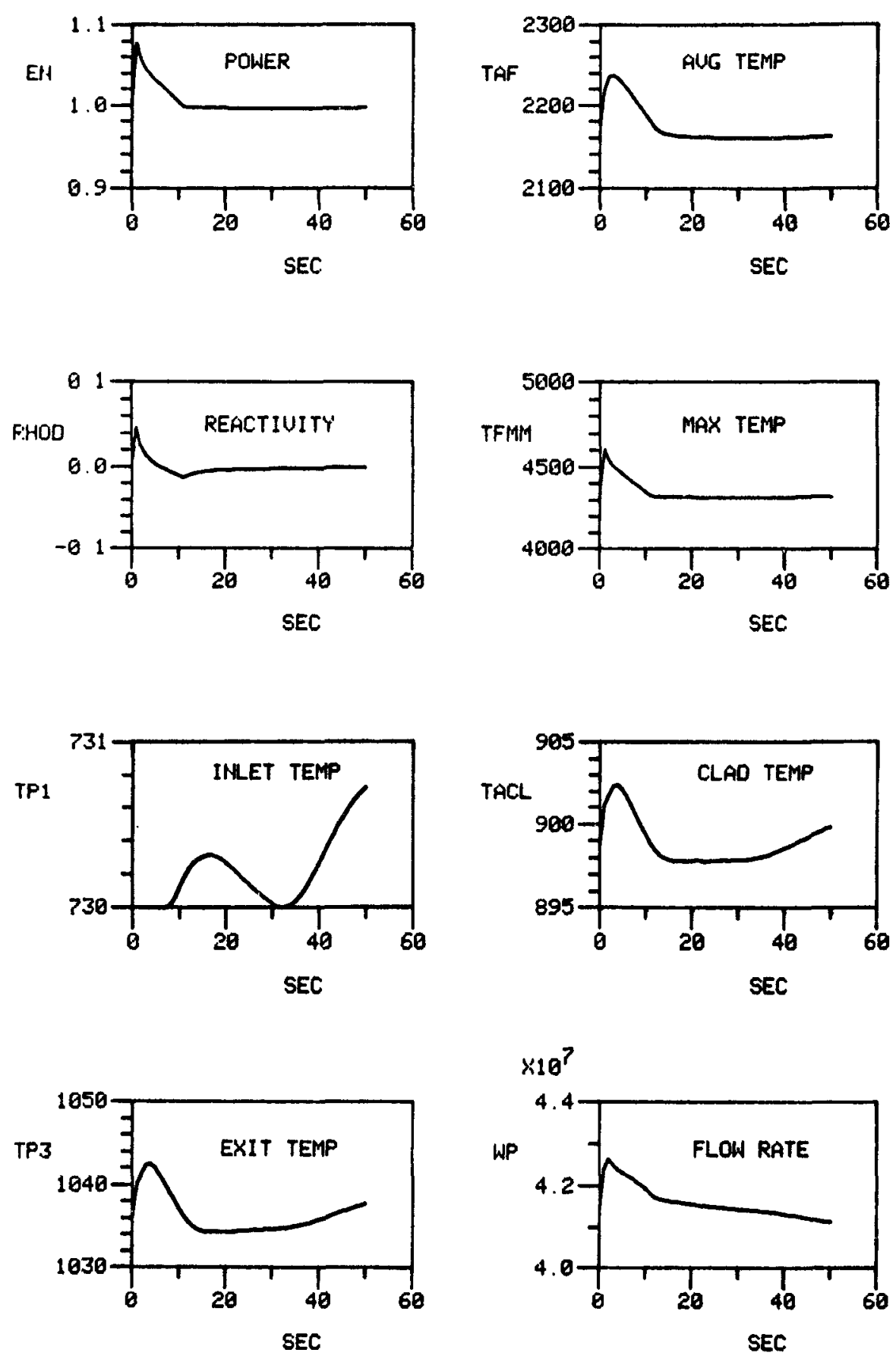

Fig. 4 Response of the CRBRP to a 10-cent Reactivity Step (Cont.). 
FLON RATES

$\times 10^{7}$

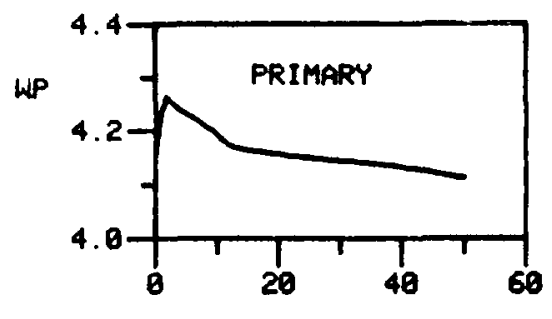

SEC

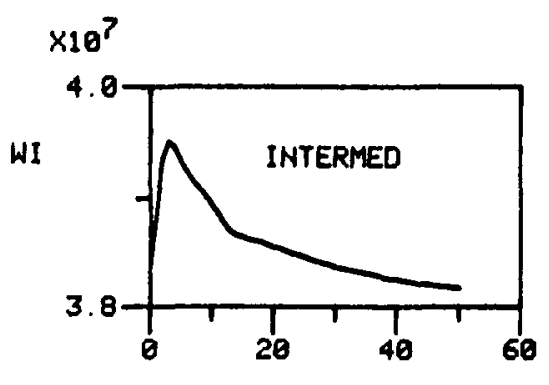

SEC

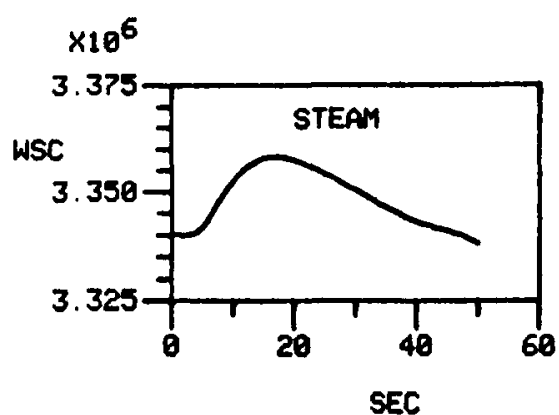

SEC

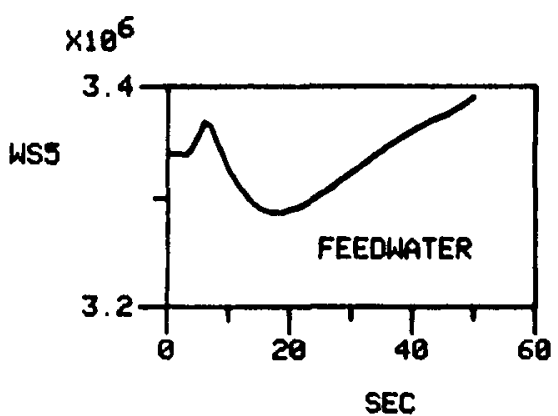

INTERMEOIATE LOOP SODIUM TEMPERATURES

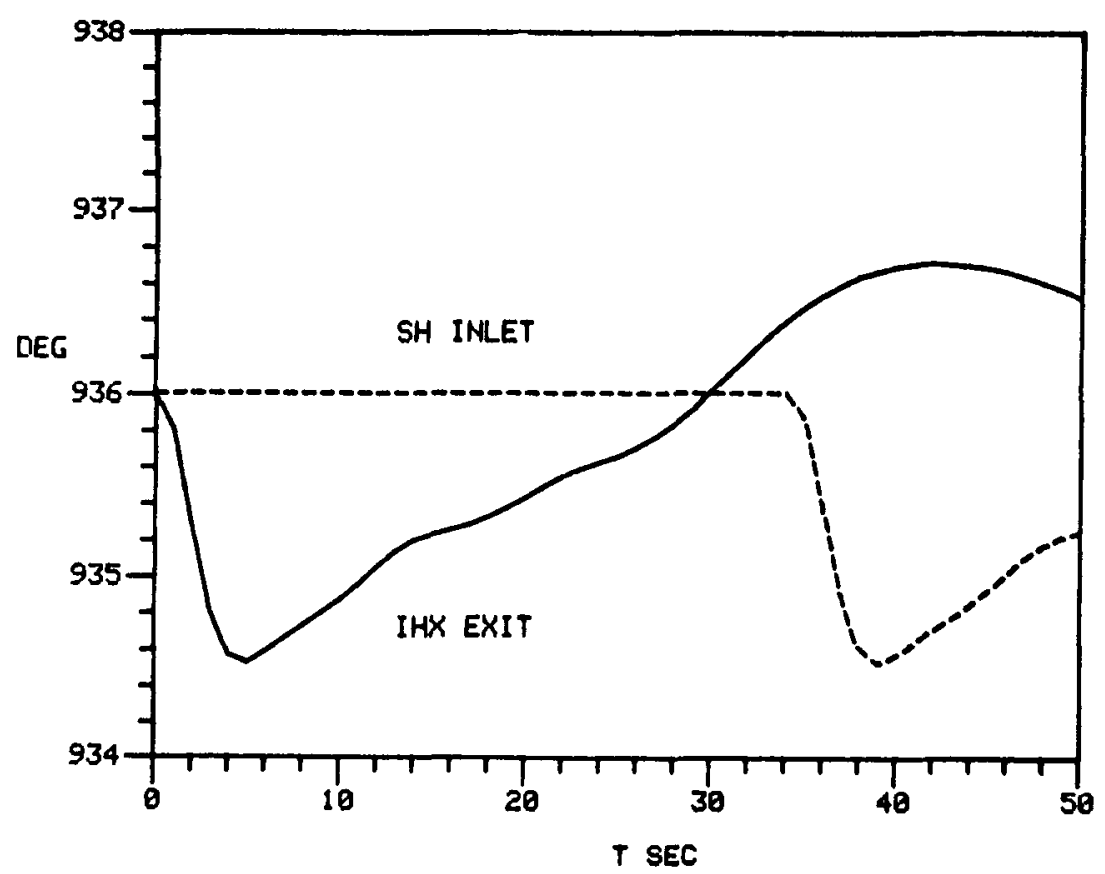

Fig. 5. Response of the CRBRP to a 10-cent Reactivity Step (Cont.). 


\section{REFERENCES}

BYRNE, G. D., and HINDMARSH, A. C. (1975): "A Polyalgorithm for the Numerical Solution of Ordinary Differential Equations", ACM Transactions on Mathematical Software, Vol. 1, No. 1, p. 71.

GEAR, C. W. (1971): Numerical Initial Val.ue Problems in Ordinary Differential Equations. Prentice-Hall, Inc.

HETRICK, D. L., SHINAISHIN, M. A., and WAIT, J.V. (1978): "Application of the DARE Simulation System to Nuclear Power Plant Dynamics", Proceedings of the Second International Symposium on Large Engineering Systems, G. J. Savage and P. H. Roe (eds). Sandford Educational Press.

JACOBI, W. M. (1974): "The Clinch River Breeder Reactor Project Nuclear Steam Supply System", Nuclear Engineering Internationa1, Vo1. 19, No. 221, p. 846 .

KORN, G. A., and WAIT, J. V. (1978): Digital Continuous System Simulation. Prentice-Hall, Inc.

SHINAISHIN, M. A. (1976): "Dynamic Simulation of a Sodium-Cooled Fast Reactor Power Plant", Ph.D. Dissertation, University of Arizona; Report No. NUREG-0110. 
APPENDIX A

Glossary of Variable Names Used in BRENDA

The following is an alphabetical list of variable names used in this version of BRENDA. Figure 1 should be consulted for a key to locations in the plant; for example, TSC is the steam temperature at the superheater exit, HS5 is the feedwater enthalpy, etc. Region averages are generally signified by using $A$ as the second letter; for example, TAWA is the average superheater wall temperature, PAS2 is the two-phase pressure in the evaporator, etc.

This glossary was prepared for an uncontrolled version of the plant model, so that many of the variables and parameters in the controller models are not included. The controllers can generally be understood by noting that the names of gains begin with the letter A, error signals with $\mathrm{E}$, and time constants with $\mathrm{T}$. The names of measured values end with the letter $M$, and the names of demand values end with $D$. Hence, for example, in the turbine valve controller we have gain factors such as AEPT, equations such as

$$
\text { EPS9 = PS9D - PS9M, }
$$

and time constants such as TSV9. For details, see the dissertation by Shinaishin (1976). 
a

A 11 T

A $12 \mathrm{~T}$

A $21 \mathrm{~T}$

A $22 \mathrm{~T}$

AI

AII?

A IP P

$\mathrm{AKF}$

AKE W

ALF 3

AIF A2

A 1

Av9

$\mathrm{A} \times \mathrm{ECC}$

B

B 1T

E2?

SD

BDOB

BETA

C

CDMP

CHEP

CPS

C 59

CV 9

D

$01-56$

D1. $-D 6$.

$D C$

5

DEMSL LBM/SEC

DEYSS LOM/HR

DETERK

DHE STO/LBH-PSI

DHFP2 3TU/LBM-PSI

วHG $3 T \mathrm{H} / \mathrm{LBH}-\mathrm{PSI}$

DHGP2 BTU/LBM-PSI
GROUPING OF TERYS USED IN CAICJLATING DPAS2

GROUPING OF TREMS USED IN CALCULATING JETERM AND WS 3DD

GROUPING OF TERMS OSEE IN CALCULATING DETERM AND WSTDD

GROUPING OF TERMS OSED IN CALCULATING DET SRE AND IIS3DD

GROUPING OF TERMS USED IN CALCULATING

DETERM BND HS 1DD

AEMATURE CURRENT

INTERMEDIATE PUMP INERTIA

PRIMARY PUAP INERTIA

FOEL THERMAL CONDUCTIVITT

PUEI THERMAL CONDOCIIVITY

VOID FRACTION AT NODE 53

AVERAGE VOID FRACTION IN REGION AS2

EFFECTIVE STEAM CROSS SECTION AT NODE S1

CONSTANT USED IN CALCULATING PVG

AXIAL EXPANSION COEFFICIENT

GROUPING CF TERMS USED IN CALCULATING

DLS 1

GROUPING OP TZRMS USED IN CALCULATING

VIS 1DD AND WS3DD

GROUPING OF TERMS USEL IN CALCJLATING

US 1DD AND WS3DD

SUL OF THE PRODUCTS OF THE INEIVIDOAL

DEIAY GROUP NOELAIIZED CONCENTRATIONS MOLTIPLIED BY THEIE INDIVIDOAI DELAY

GROUP PRACTIONS

$B D / B E T A$

TOTAL FRACTION OF DELAYED NEUTRONS

GROUPING CF TERMS OSED IN CALCULATING

\section{DIS 1}

DUMP FLON CONSTANT

CHANGE OF MOMENTUM PRESSURE ITEAD

STEAM SPECIZIC HEAT IN REGION ASA

CONSTANT USED IN CALCULATING PS9C

CONSTANT USED IN CAICULATING PS9SC

A FACTOE APQEARING IN DEROMINATOR

OF 2 AS4 DIFFERENTIAL ZQJATION

NORUALIZED CONCENTRATION OF ITH DELAT

SROUP

TIME DERIVATIVES OF D 1-D6

DOPQLER CONSTANT

TIME DERIVATIVE OF EMS 4

TINE DERIVATIVE OF EMS5

DET BRMINENT USED IN CALCULATING HSIDD

AND $\exists$ S3DD

FONCT FON SUBEOUTIYE NAICH CALCULATES THE

DERIVATIVE OF THE SATURATED HATEE

ENTHALPY VITH RESPECT TO PRESSORE IN THE

ZVAPORATER

DERIVATIVE OF SATURATED WATER

ZNTHALPY UITH RESEECT TC EAS2 AT NODE S2

FONCTION SOBROUI INE 2 HICH CALCULATES IHE

DEEIVATIVE OF IHE SAIURATED STEAM

ZNTHALPY WITH RESPECT TO PRESSURE IN THE

ZV APORATER

JERIVATIVE OF SATURATEL STEAM

ZNTHALPY WITH RESPECT TO ZASL AT NODE S2 


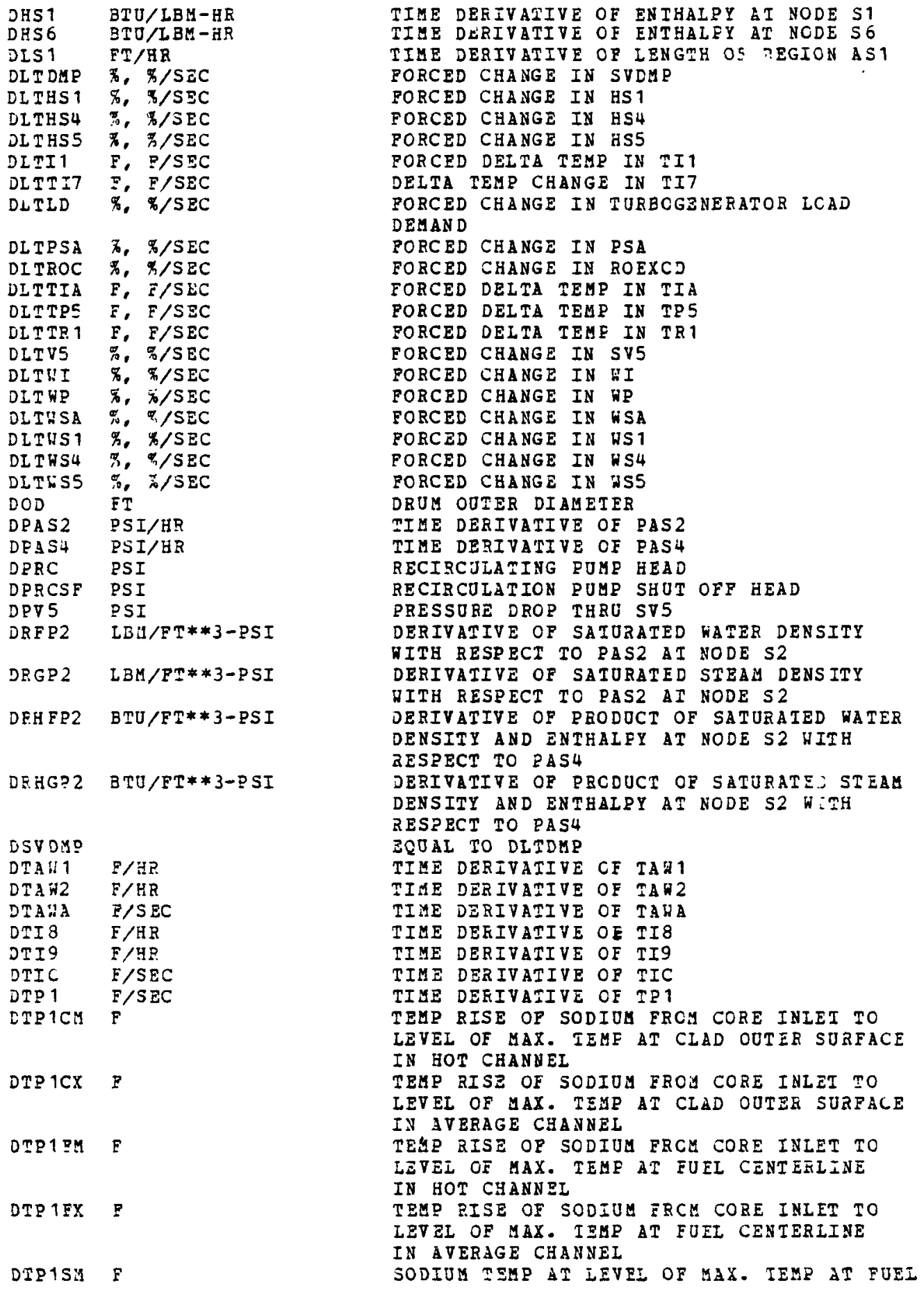




\begin{tabular}{|c|c|}
\hline DTP 1SX & $\mathbf{F}$ \\
\hline DTPC. & $\mathbf{F}$ \\
\hline DTPCMX & $\mathbf{F}$ \\
\hline $\begin{array}{l}\text { DTSC } \\
\text { DV } \\
\text { DVF }\end{array}$ & $\begin{array}{l}\text { I/SEC } \\
\text { FT**3 } \\
\text { FT**3/L3M-PST }\end{array}$ \\
\hline DVG & $F T * 3 / \mathrm{LBH}-\mathrm{PSI}$ \\
\hline $\begin{array}{l}\text { DWDMRS } \\
\text { DWIS } \\
\text { DWIST } \\
\text { DWPS } \\
\text { DWPST } \\
\text { DWS10 } \\
\text { DHS 3D } \\
\text { DWSSS } \\
\text { DWSAS } \\
\text { DWSASD }\end{array}$ & $\begin{array}{l}\text { L } 3 M / S E C \\
L B M / S E C * * 2 \\
L B Y / S E C * * 2 \\
L B M / S E C * * 2 \\
L B M / S E C * * 2 \\
L B M / S E C * * 2 \\
L 3 U / S E C * * 2 \\
L B H / S E C * * 2\end{array}$ \\
\hline $\begin{array}{l}\text { E } \\
\text { ELS } 1 \\
\text { ELS } 1 . \\
\text { EIS } 2 \\
\text { ELS } 5\end{array}$ & $\begin{array}{l}K V \\
F T \\
\text { FT/SEC } \\
\text { FT } \\
F T\end{array}$ \\
\hline ELS 7 & $\mathrm{FT}$ \\
\hline $\begin{array}{l}\text { ELS } 31 \\
\text { EIS } 32 \\
\text { ELS61 } \\
\text { EIS } 62 \\
\text { ELS63 } \\
\text { ELS64 } \\
\text { ELSC1 }\end{array}$ & $\begin{array}{l}\text { FT } \\
\text { FT } \\
\text { FT } \\
\text { FT } \\
\text { PT } \\
\text { FT } \\
\text { FT }\end{array}$ \\
\hline EISC 2 & FT \\
\hline ELSAT & $E T$ \\
\hline $\begin{array}{l}\text { BUS } 4 \\
\text { EMS UD } \\
\text { EUS } 4 I \\
\text { BMS S } \\
\text { EMSS. } \\
\text { EMS SI } \\
\text { EMW }\end{array}$ & $\begin{array}{l}\text { LBM } \\
\text { LBM } \\
\text { LBI } \\
\text { LBY } \\
\text { LBI/SEC } \\
\text { LSY } \\
\text { SEC VS. }\end{array}$ \\
\hline $\begin{array}{l}\text { EMHD } \\
\text { SMHI } \\
\text { ENHM }\end{array}$ & $\begin{array}{l}Y H \\
Y H \\
Y H ̈ H\end{array}$ \\
\hline
\end{tabular}

SOPPACE IN HOT CAANALI

TELP RISE OF SODIUA FROA CORE INLET TO

LEVEL OF MAX. TEUP AT FUEL SURFACE

IN AVERAGE CHANNEL

DELTA TEMP BETWEEN CLAD CUTER SURFACE AND SODIUM AT IEVEL OF MAX. CIAD OUTER

SJRFACE TEMP IN HOT CHANNEL

DELTA TEMP BETREEN CLAD OUTER SURFACE AND

SODIOM AT LEVEL OF VAX. CLAD OUTER

SUEFACE TEMP IN AVERAGE CHANNEL

TIME DERIVATIVE OF TSC

STEAM DRUE TOLUAE

PONCTION SOBRODTIRE RHICH CALCUIATES THE SATORATED MATER SPECIEIC VCIOUE WITH RESPECT TO PRESSURE IN THE EVAPORATER FUNCTION SUBROUTIAE WHICH CALCULATES THE SATURATED STEAM SPECIFIC VOLUME HITH RESPECT TO PRESSURE IN THE EVAPORATER STEP CHANGE IN TURBOGENERATOR DUAP FION TIME DERIVATIVE OF WIS DWIS MULTIRLIED BI TAUHI

TIME DERIVATIVE CE WPS

DW QS WULTPLIED EY TAONP

TIME DERIVATIVE OF HS 1D

TIME DERIVATIVE OF WS3D

TIUE DERIVATIVE OF WSSS

TIME DERIVATIVE OF WSAS

TIME DERIVATIVE OF USAS FOR A CLOSED LOOP

STATOR E.M.E.

LENGTH OF REGION AS 1

TIGE DERIVATIVE OF ELS 1

LENGTH OF REGION AS2

LENGTH OF PIPE LINE BETNEEN WAIN

FEEDW ATER HEADER ALD NODE S5

LENGTH OF PIPE IINE BETHEEN NCDE S7 AND NODE SA

PIPE LEISTH OP RISER OF DIAEETER \#1

PIPE LEYGTH OF RISER OF DIAUETER $\# 2$

PIPE LENGTH CF DONNCOHER OF DIAMETER 1

PIPE LENGTH OF DOWYCO

PIPE LENGIH CF CCANCOHEF CF DIAUETER 3

PIPE LENGTH OF DONNCOBER OF DIAUETER 4

LENGTH OF PIPE LINE BETHEEN NODE SC AND SOPERHEATED STEAM GEADER

LENGTH OP PIPE LINE SETHEEN SUPERHEATED

STEAL HEADER AND NODE S9

TOTAL LENGTH OF SUEERHEATER AND

EV APORATOR TUEES (IDENTICAL ONITS)

MASS OF SATURATED STEAM IN SIEAM DROM

BMS4 DELAYED BY $0.1 \mathrm{SEC}$

INITIAL EMS4

MASS OF SATURATED GATER IN STEAM DRUM

TIHE DERIVATIVE OF EMSS

INITIAL ZUSS

TORBOGENERATOR LOAD DEMAND TAEIE AS A

FUNCTION OF TIME

TORBOGENERATOR LOAD DEMAND

INITIAL TORBOGENERATOR LCAD DEMAND

MANOAL SETTING OF TURBOGENERATCR LOAD DEMAND 
EN

ENDMP

ENE

ENEVAP

ENHS 1

ENHS5

EN I

ENIP RP

ENIP. RRY/SEC

ENL

ENLD

ENMWU

ENP

ENP P

ENPP.

ENP SA

ENROC

ENS

ENS94

ENSC2

ENSSC

ENSUB

ENTI1

ENTI7

ENTIA

ENTP5

ENTR1

ENV 5

ENTI

ENU P

ENWS1

ENUS4

Exis5

ENUS6

ENVS7

ENM SA

EPS

ETA IP

ETAPD

EXAI

F143

BTO/AR

$1 / R A D$

$2 S F-5 E C * * 2 / \operatorname{LB} * * 2$

FETAG DEGREES

FETAG. DEGREES/SEC

PEC

FPGC

$F P C$

$2 S I /(L B I / S B C) * * 2$

FRPH IBF/PT**2

PRPH 34 LBF/FT**2

FS1

FSIA PSI-SEC**2/IB\#**2-PT

FS2
MORMALIZED REACTOK FOVEK LEVEL

SWITCH PARAUETER FOR SVDMP AND DSVDMP

NOUBER OF EVAFCRATCE TOBES

NOMBER OF EVAPORATORS

SUITCH PARAMETER FCE HS 1

SWITCH PARAMETER FOR HSS

NOUBER OF TUBES IN IAX

INTERHEDIATE ROEP SPEED

TIUE DERIVATIVE OE ENIP

NOABER OF HEAT TRANSPORT LOOPS IN THE

RLANT

SHITCH PARAMETER FOR AI AND EMWD

SRITCE PARALETEE FCE EHHM

NUEBER OF EUEL PINS IN THE CORE

PRIUARY PUUP SPEED

TIUE DERIVATIVE OF ENPQ

SHITCE PARAMETER FOK PSA

SHITCH PARALETER FOR ROEXCD

NUMBER OF TUBES IN THE SUPERHEATER

NOLBER OF TURBINE CONTROI VALVES AT

NODE $S 9$

NUMBER OF PIRE IIYES BETWEEV SOPERLEATED

STEAM HZADER ANE NODE S9

SWITCH PARAGETER FOR SHORT CIÉCUIT

NOMBER OF FUEL SUBASSEMELIES IN THE CORE

SHITCE COHTROLIING TI 1

SAITCH PARAUETEE POF TI7

SHITCH PARAGETER FOR TIA

SHITCH PARAMETER FOA TP5

SHITCA PARAIETER FOR TR 1

SHITCH PARAHETER FOR SV5

SHITCH PARAMETER FOE UI AND DHIS

SRITCH PARAUETER FCE HP AND DHFS

SHITCH PARAKETER FOR NS1

SWITCH PARAMETEE ROE WS 4

SWITCH PARAMETER FOR WS5

SWITCH PARAMETEF FOR WS6

SHITCH PARAMETER FOE IST

SWITCH PAPAMETER FCF NSA AND CUSAS

RATED REACTOR THER LAL POWER

EEFICIENCT OF INTERUELIATE RUMP

EFFICIENCY OF PRIGARY PUMP

DAMPING FACTOR

PRESSURE LOSS COEFFICIENT FOR INLET

NOZZLES OF FUEL SOEASSEMBLIES, SHIELD/

ORIPICE, ROD BONDLE, AND FOEL

SOBASSEMBL IES LUHPED INTO ONE TERM

PORER DNGLE

TIME DERIVATIVE OF FETAG

FRACTION OE SODIOA RASSING THRO CORE

FRACTION OP POHER GENERATED IN THE CORE

RECIRCOLATION POAP FRICTION COEF.

PRICTION PRESSORE HEAD IN THE

EVAPORATOR/STEAL DRUM LOOP

FRICTION PRESSORE HEAD IN THE RISER

ERICTION FACTOR IN REGION AS1 OBTAINED

PEOA EQUATIONS DESCEIEIMG MOODY CHART

PRESSURE LOSS COEFEICIENT PER FOOT IN

REGION AST

FRICTION FACTOR IN REGICN AS2 OBTAINED 


\begin{tabular}{|c|c|c|}
\hline 524 & $2 S I-S E C * * 2 / L 3 y * * 2-P T$ & $\begin{array}{l}\text { FROA EQUATIONS DESCRIBING YOODY CHART } \\
\text { PRESSURE LOSS COEFFICIENT PER FOOT IN } \\
\text { REGION AS2 }\end{array}$ \\
\hline \$5 & & $\begin{array}{l}\text { FRICTION FACTOR IN THE FEEDWATER PIPE } \\
\text { LINES OBTAINED FROM EQOATIONS DESCRIBING } \\
\text { MOODY CHART }\end{array}$ \\
\hline SC 1 & $\begin{array}{c}\mathrm{L} 3 \mathrm{~F} / \mathrm{FT} * * 2 /(\mathrm{LBH} / \mathrm{SEC}) * * 2 \\
\text { PSI }-\mathrm{SEC} * * 2 / \mathrm{LBM} * 2\end{array}$ & $\begin{array}{l}\text { FRICTION FACTOR IN THE COWNCOMER } \\
\text { PRESSURE LOSS COEFFICIENT BETWEEN NODE SC } \\
\text { AND SURERHEATED STEAM HEADER }\end{array}$ \\
\hline $5 \mathrm{C} 2$ & PSI-SEC**2/LBU**2 & $\begin{array}{l}\text { PRESSURE LOSS COEFFICIENT BETWEEN } \\
\text { SUPERHEATED STEAU HEADEK AND TURBINE }\end{array}$ \\
\hline 85 & $\begin{array}{l}\mathrm{PSI} /(\mathrm{IBM} / \mathrm{SEC}) * * 2 \\
\text { BTO/LBM } \\
\text { BTU/LBU }\end{array}$ & $\begin{array}{l}\text { FRICTION FACTOR FOR SV5 } \\
\text { AVERAGE ENTHALPY IN REGION AS } \\
\text { AVERAGE ENTHALPY IN REGION ASA }\end{array}$ \\
\hline & $F-F T * 2$ & $\begin{array}{l}\text { SCDIUU FILM HEAT TRANSFER COEFEICIENT } \\
\text { IN THE CORE }\end{array}$ \\
\hline DG & $B T U / H R-F-F T * 2$ & PUEL GAR CONDUCTANCE \\
\hline & FT & $\begin{array}{l}\text { HEAT TRANSFER COEFEICIENT PER FOOT } \\
\text { BETWEEN MIDWALI OF REGION AWI AND REGION } \\
\text { AS1 }\end{array}$ \\
\hline $\operatorname{bs} 2$ & $\mathrm{BTU} / \mathrm{HR}-\mathrm{P}-\mathrm{FT}$ & $\begin{array}{l}\text { HEAT TRANSPBR COEFEICIENT PER FOOT } \\
\text { BETH EEN MIDWALL OF REGION AN } 2 \text { AND KEGION } \\
\text { AS2 }\end{array}$ \\
\hline DSPH & $L B F / F I$ & $\begin{array}{l}\text { HYDRO-PRESSURB HEAD IN THE EVAPORATER/ } \\
\text { STEAM DRUH LOOP }\end{array}$ \\
\hline DW 1 & $B T O / B R-F-F T$ & $\begin{array}{l}\text { HEAT TRANSFER COEFEICIENT PER FOOT } \\
\text { BETWEEN MIDHALL OF REGION AWI AND REGION } \\
\text { AI8 }\end{array}$ \\
\hline & F-FT & $\begin{array}{l}\text { HEAT TRANSFER COEFFICIENT PER FOOT } \\
\text { BETWEEN MIDHALL OF REGION AW2 AND REGICN } \\
\text { AI7 }\end{array}$ \\
\hline $1 E X$ & BT U/LDH & ENTHALPY AT EXEAOST OF TURBINE \\
\hline & i. BTL & $\begin{array}{l}\text { ENTHA LPY OF SATURATED WATER TABLE } \\
\text { AS A FONCTION OP PRESSURE }\end{array}$ \\
\hline FS & $\mathrm{BTO} / \mathrm{LBZ}$ & ENTHALPY OF SATURATED HATER AT NCDE S2 \\
\hline G & PSI VS. BTU/LBM & $\begin{array}{l}\text { SATURATED STEAM ENTHALPY TABLE AS A } \\
\text { FUNCTION OF PEESSORE }\end{array}$ \\
\hline GS & L BMI & ENTHAIPY OF STEAM AT NCDE S2 \\
\hline$+I$ & $\begin{array}{l}\text { BTO/LBM } \\
\mathrm{BTJ} / \mathrm{LBM}\end{array}$ & $\begin{array}{l}\text { ENTHAIPY OF SATORATED STEAI AT REGION } 54 \\
\text { INITIAL HGS4 }\end{array}$ \\
\hline$T$ & $3 \mathrm{TO} / \mathrm{HR}$ & OVERALL HEAT TRANSFER COEFFICIENT BETHED \\
\hline 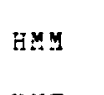 & RPM VS. $10 * * 3 G P$ VS. & $\begin{array}{l}\text { THE PRIMARY AND SECCNDARY SODIUM IN ILX } \\
\text { GEAD OF PUMP TAELE AS A FUNCTICX CF THE } \\
\text { PUMPS SPEED AND ELOW }\end{array}$ \\
\hline 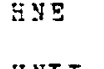 & 3 & $\begin{array}{l}\text { SODIUU FILU HEAT TRANSFER COEFFICIENT } \\
\text { IN REGIONS AI7 AND AI8 }\end{array}$ \\
\hline [N] & $0 / \mathrm{h}$ & $\begin{array}{l}\text { SECONDARY SODIOY FIIY HEAT TRANSFER } \\
\text { COEFFICIENT IN IHX }\end{array}$ \\
\hline HNI & 2 & $\begin{array}{l}\text { PRIMARY SODIUM FIL A AEAT TRANSFER } \\
\text { COEFFICIENT IN IHX }\end{array}$ \\
\hline HiN & $U / \bar{i}$ & $\begin{array}{l}\text { SODIUL FILA HEAT TABNSEEA COEFFICIENT } \\
\text { IN REGION AIA }\end{array}$ \\
\hline OI $R$ & $\begin{array}{l}\text { ET } \\
\text { FT }\end{array}$ & $\begin{array}{l}\text { HEAD OF INTERMEDIATE PUUP } \\
\text { HEAD OP PRIUARY POME }\end{array}$ \\
\hline 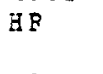 & & $\begin{array}{l}\text { OVERALL GEAT TRANSFER COEFFICIENT BETWEE } \\
\text { FUEL AND SODIUU IN THE CORE }\end{array}$ \\
\hline Bh & $3 T$ & $\begin{array}{l}\text { OVERALI HEAT TFANSFER COEFEICIENT PER } \\
\text { FOOT BETHEEN FOEL CENTERIINE ANE SCDIUA } \\
\text { IY THE CORE }\end{array}$ \\
\hline & $B T U / 4 P-\vec{r}-\vec{F} T$ & $\begin{array}{l}\text { OVERALI HEAT TRANSFER COEFFICIENT RER } \\
\text { FOOT BETWEEN FUEL SUREACE AND SODIUM IN }\end{array}$ \\
\hline
\end{tabular}




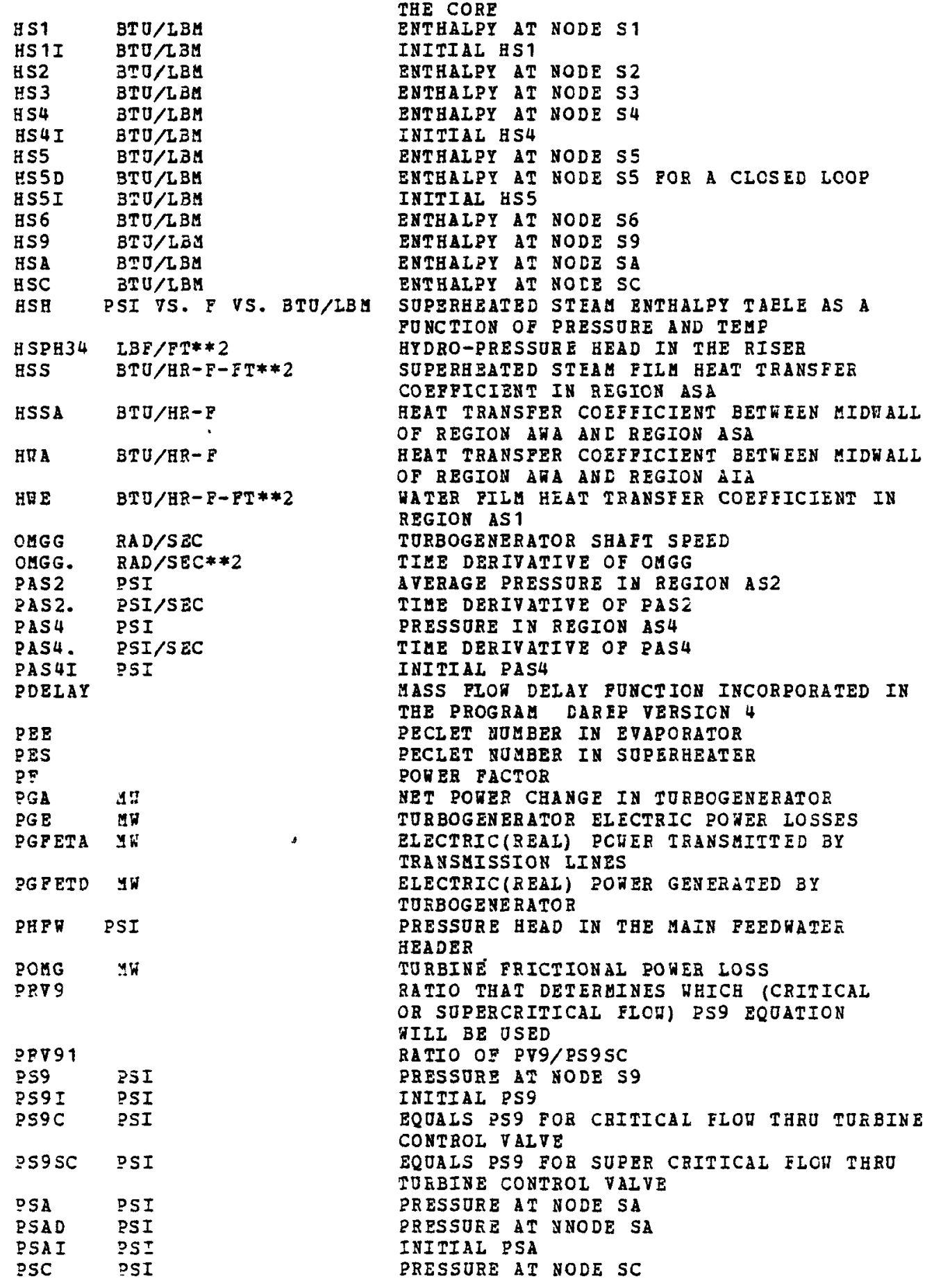




\begin{tabular}{|c|c|}
\hline $\begin{array}{l}\text { PSS } \\
\text { PT } \\
\text { PT. } \\
\text { PV9 } \\
\text { QC }\end{array}$ & $\begin{array}{l}P S I \\
H W \\
Y W / S E C \\
P S I \\
B T U / H R-E T * * 3\end{array}$ \\
\hline QIONI & $10 * * 3$ GALLONS/REV \\
\hline $\begin{array}{l}\text { QIP } \\
\text { QPONP }\end{array}$ & $\begin{array}{l}10 * * 3 \text { GP } 4 \\
10 * 3 \text { GALLONS/REV }\end{array}$ \\
\hline $\begin{array}{l}\text { QPP } \\
\text { R } \\
\text { FD PSI }\end{array}$ & $\begin{array}{l}\text { 10**3 GPM } \\
\text { VS. QOALITY VS. F.H. }\end{array}$ \\
\hline $\begin{array}{l}\text { EECC } \\
\text { FES } \\
\text { RES1 } \\
\text { RES2 } \\
\text { EHOAS1 } \\
\text { RHOAS2 } \\
\text { PHOASA } \\
\text { PHOCD } \\
\text { EHOCDI } \\
\text { RHOD } \\
\text { PHOFEC } \\
\text { RHOFBD } \\
\text { EHOFS2 } \\
\text { RHOFS4 } \\
\text { PHOGS2 } \\
\text { PHOGS4 } \\
\text { PHOIE } \\
\text { RHONA }\end{array}$ & 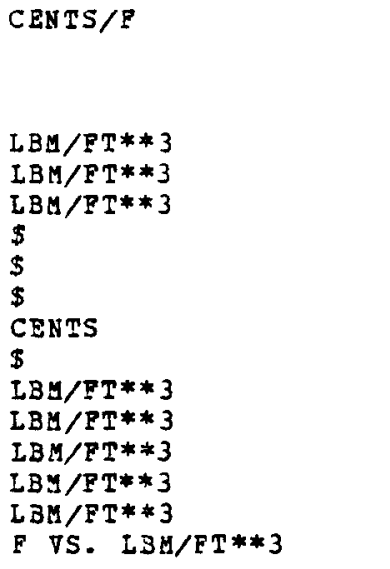 \\
\hline $\begin{array}{l}\text { RHOS3 } \\
\text { RHOSC } \\
\text { RHOII1 } \\
\text { RHOTI3 } \\
\text { RHOTIC } \\
\text { RHOTP1 } \\
\text { RHOMP3 } \\
\text { RHOTP4 } \\
\text { RHOTR1 } \\
\text { RHOTR3 } \\
\text { RHOTS6 } \\
\text { RHOW }\end{array}$ & 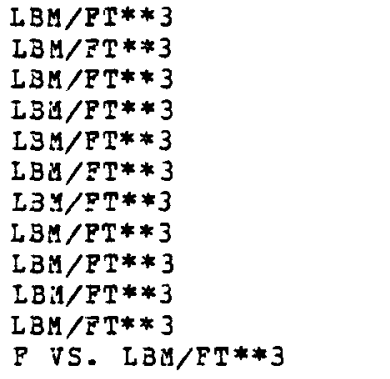 \\
\hline $\begin{array}{l}\text { ROEXCD } \\
\text { ROFBCI }\end{array}$ & $\begin{array}{l}\$ \\
\text { CENTS }\end{array}$ \\
\hline $\begin{array}{l}\text { ROTAI1 } \\
\text { FOTAIA } \\
\text { ROTAP1 } \\
\text { ROTAP5 }\end{array}$ & 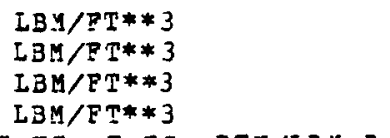 \\
\hline S9 PSI & I VS. $E 75 . \quad 3 T U / L B M-E$ \\
\hline $\begin{array}{l}\text { SAI } \\
\text { SEXH } \\
\text { SHS } \\
\text { SHS } 4 \\
\text { SHS } 5\end{array}$ & $\begin{array}{l}B T U / I B M-R \\
S E C \\
S E C \\
S E C\end{array}$ \\
\hline
\end{tabular}

ADERAGE STEAM PRESSURE IN SURERHEATER

TUPBINE POWER ACTOATING THE SHAFT

IIME DERIVATIVE OF PT

PRESSURE AT YODE $Y 9$

VOLOMETRIC HEAT GENERATION AT CENTER OF

POEL PIN IN THE AVERAGE CHANNEL

INTERUEDIATE PUMP FLOW DIVIDED BY

INTERUEDIATE PUMF SPEED

FION RATE OF INTERMEDIATE PUMP

PRIYARI PUMP FIOH LIVIDED BY PRIMARY

POUP SPEED

PLOW RATE IN PRIMARY PUMP

2 PHASE FIOW FRICTION MULTIPLIER

FRICTION MULTIPLIEE TAELE FOR REGION

AS2 AS A FUNCTION OF RRESSURE AND QUALITY

RADIAL EXPANSICN CCEFFICIENT

REY XOLDS NOHBER IN SUPERHEATER

REYNOLDS NUMBER IN REGION AS 1

REY NOLDS NUMBER IN REGION AS2

AVERAGE DENSITY IN REGION AS1

AV ERAGE DENSITY IN EEGION AS2

ATERAGE DEISITY IN REGION ASH

CONTROL ROD REACTIVITY

INITIAL RHOCD

TOTAL CORE REACTIVITY

TOTAL REACTIVITI FEEDBACK

TOTAL REACTIVITY FEEDBACK

DENSITY OP SATUPATED HATER AT NODE S2

DENSITY OF SATURATED WATER IN REGION AS4

DENSITY OF SATURATEL STEAM AT NODE $S 2$

DENSITY OF SATUPATED STEAH IN REGION AS4

AVERAGE SODIOU DENSITY IN EVAPORATOR

DENSITY OF SODIOM TABIE AS A FUNCTION

OF TEMP

DENSITY AT NODE 53

DENSITY AT NODE SC

DENSITY AT NODE IT

DENSITY AT NODE I3

DENSITY AT NODE IC

DENSITY AT NODE P1

DENSITY AT NODE P3

DENSITY AT NODE P4

DENSITY AT YODE E1

DENSITY AT NODE R3

JENSITY AT NODE S6

DENSITY OF SATUEATED VATER TAELE AS A

FU HCTION OF TEMP

ZCCENTRIC CONTROL ROD BEACTIVITY

REACTIVITY FEEDBACE DDE TO AYERAGE FUEL

TEUPEPATURE AT COLD STANDBI CONDITIONS

DENSITY IN REGION AIT

DENSITY IN REGION AIA

DEUSITY IV REGION API

DENSITY IN REGION APS

SUPERH EATED STEAM ENT FOPY TABLE AS A

EUNCIION OF PRESSURE AND TEMP

RATIO OF STEAH/WATER DENSITIES AT NODE $S 2$

ENTROPY AT EXHAUST OF TOEBINE

TIME AT END OP RAMP CEANGE IN HST

TIYE AT END OF RAMP CHANGE IN HS

TIME AT END OE RAKP CHANGE IN HSS 


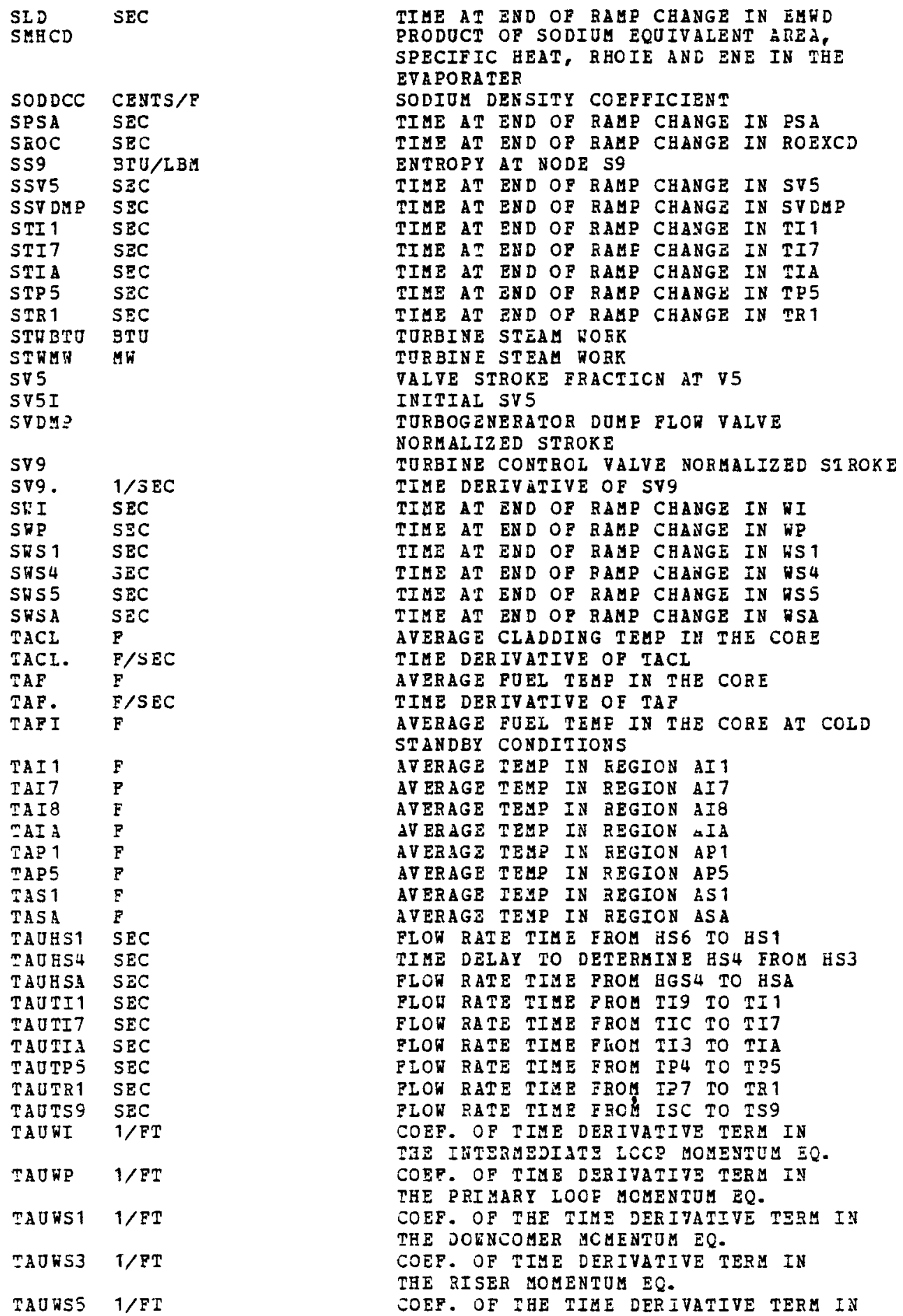




\begin{tabular}{|c|c|}
\hline TAUWSA & $1 / \mathrm{FT}$ \\
\hline $\begin{array}{l}\text { TAH } 1 \\
\text { TAW1. } \\
\text { TAN } 2 \\
\text { TAN2. } \\
\text { TAMA } \\
\text { TANA. } \\
\text { TCMAX }\end{array}$ & $\begin{array}{l}F \\
E / S E C \\
F \\
F / S E C \\
E \\
E / S E C \\
F\end{array}$ \\
\hline TCM & $F$ \\
\hline $\begin{array}{l}\text { TFC } \\
\text { IFK } \\
\text { TFMAX }\end{array}$ & $\begin{array}{l}\mathrm{C} \\
\mathrm{K} \\
\mathrm{F}\end{array}$ \\
\hline IFMU & $F$ \\
\hline TFR & $S E C$ \\
\hline 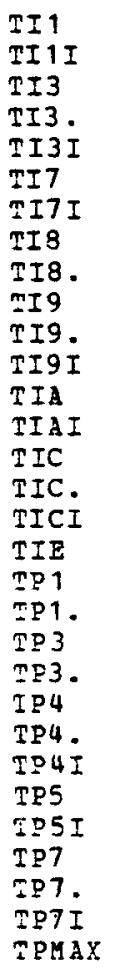 & $\begin{array}{l}F \\
F \\
F \\
F / S E C \\
F \\
F \\
F \\
F \\
F / S E C \\
F \\
F / S E C \\
F \\
F \\
F \\
F \\
F / S E C \\
F \\
F \\
F \\
F / S E C \\
F \\
E / S E C \\
F \\
F / S E C \\
F \\
F \\
F \\
F\end{array}$ \\
\hline TPML & $\mathbf{F}$ \\
\hline$T P Y C M X$ & $F$ \\
\hline TPY FMX & $F$ \\
\hline & F \\
\hline
\end{tabular}

THE PEEDNATER LOCP MOMENTUM EQ.

COEF. OP IHE TIME DERIVATIVE TERM IN

SOPERHEATER MOMENTUE EQ.

TEMP OF EVAPORATOR HALI IN REGICN AW 1

TIME DERIVATIVE OF TAW 1

TEMP OF EVAPORATCR WALL IN REGTON AH 2

TIME DER ITATIVE OF TAH2

TEMP OP SUPEREEATER WALL IN BEGION ANA

TIUE DERIVATIVE OF TA HA

MAXIMUU CIAD OUTER SURFACE TEMP IN

THE AVERAGE CHANNEL

YAXIMOU CLAD OUTER SOREACE TEUP IN

THE HOT CHANAEL

TEMP OF FUEL

TEMP OF ROEL

MAXIUUM PUEL CENTEELINE TEMP IN TEE

AVERAGE CBANNEI

MAXIYOM FUEL CENTERLINE TEUP IN TEE

HOT CBANEEL

TIME FOR RECCVERY AFTER TRANSMISSION

IINE FAILURR

TEMP AT NODE I1

INITIAL TI 1

TEMP AT NODE I3

TIME DERIVATIVE OE TI3

INITIAL TI 3

TEMP AT NODE I7

INITIAL TI7

TEMP AT NODE I8

TIME DERIVATIVE OF TI8

TEUP AT NODE I9

TIUE DERIVATIVE OF TI9

INITIAL TI9

TEMP AT NODE IA

INITIAL TIA

TEMP AT NOUE IC

TIME DERIVATIVE OF TIC

INITIAI TIC

AVERAGE SODIUM TEMP IN EVAPORATOR

TEMP AT NODE $P 1$

TIME DERIVATIVE OF TP 1

TEMP AT NODE P3

TIME DER IVATIVE CF TP 3

TEM? AT NODE P4

TIME DERIVATIVE OE TR 4

INITIAI TP4

TEMP AT NODE P5

INITIAL TP5

INITIAL TP7

TIUE DERIVATIVE OF TP7

INITIAL TP 7

SOBIOU MAXIMUY TEAP AT EXIT CF AVERAGE

CHANNEI

SODIUM YAXIMUM TEMP AT EXIT OF HCT

CHANNEL

SODIUL TZMP AT THE LEVEL OF KAXIMUK TEMP

OF CIAD OUTER SORFACE IN AVERAGE CHANHEL SODIUM TEUP AI THE IEYEL OF MAXIMUM TEMP

OF FUEL CENTERLINE IN AVERAGE CAANNEI

SODIJY TEMP AT THE LEVEL OF MAXIMUM TEMP

OF FUEL SURFACE IN EVERAGE CHANNEI 


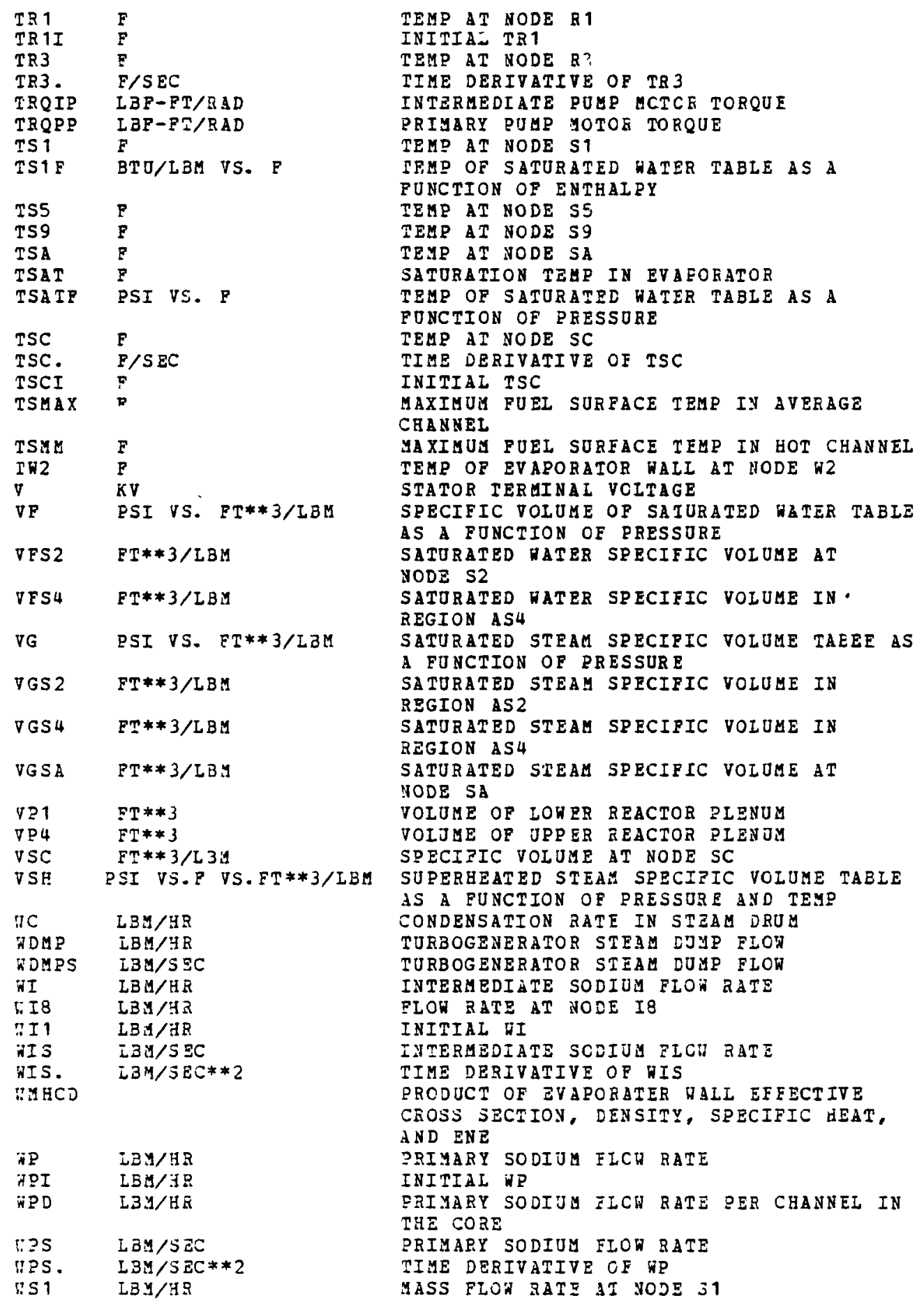




\begin{tabular}{|c|c|}
\hline $\begin{array}{l}\text { NS1D } \\
\text { BS1D. } \\
\text { WS1DD }\end{array}$ & $\begin{array}{l}\mathrm{LBH} / \mathrm{SEC} \\
\mathrm{LBM} / \mathrm{SEC} * 2 \\
\mathrm{LBM} / \mathrm{SEC} * * 2\end{array}$ \\
\hline $\begin{array}{l}\text { WS1I } \\
\text { WS1S } \\
\text { WS2 }\end{array}$ & $\begin{array}{l}\text { IBH/HA } \\
L B H / S E C \\
L B d / H R\end{array}$ \\
\hline $\begin{array}{l}\text { VS3 } \\
\text { WS3D } \\
\text { TS3D. } \\
\text { WS3DD }\end{array}$ & $\begin{array}{l}\mathrm{LBU} / \mathrm{HR} \\
\mathrm{LBU} / \mathrm{SEC} \\
\mathrm{LBM} / \mathrm{SZC} * * 2 \\
\mathrm{LBH} / \mathrm{SBC} * * 2\end{array}$ \\
\hline 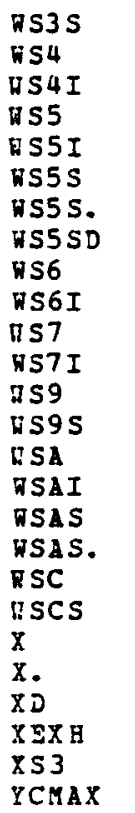 & 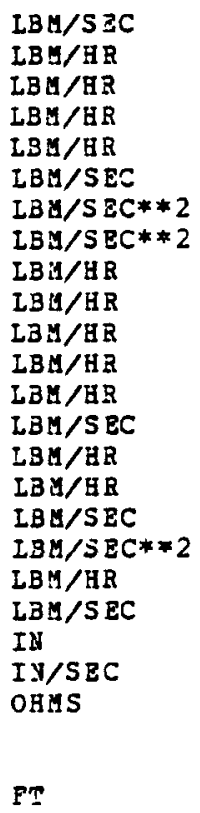 \\
\hline$\square C A M$ & $P T$ \\
\hline YCITH & $F T$ \\
\hline Y CAXTY & Fr \\
\hline IFMAX & $I T$ \\
\hline YFMII & $2 T$ \\
\hline YPATA & $E T$ \\
\hline Y PMXTH & T \\
\hline
\end{tabular}

BQOAL TO WS1S PER EVAPORATER TUBE

TIME DERIVATIVE OF US 10

EQUAL TO DWS1D FOR SWITCH FARAMETER

ENTS1 $=0.0$

INITIAL 151

MASS FLOU RATE AT NODE S1

PION RATE AT NODE S2 RELATIVE TO MOVING

BOUNDARY

MASS FIOH RATE AT NODE S3

ZOOAL TO NSZS PER EVARORATER TUBE

TIEB DERIVATIVE OF WS 3D

EQOAL TO DHS3D FOR SHITCE PARAMETER

ENTS1 $=0.0$

HASS FIOD RATE AT NODE $S 3$

MASS FLON RATE AT NODE 54

INITIAL VS 4

MASS PLOW RATE AT NODE S5

INITIAL WS

UASS FLON RATE AT HODI S5

TI $\triangle E$ DERIVATIVE OF WS5S

TIUE DERIVATIVE OF IISSS

MASS FLON RATE AT HODE 66

INITIAL WS6

MASS FLOW RATE AT NODE S7

INITIAL WS7

UASS FLON RATE AT NODE S9

IASS PLON RATE AT NODE S9

MASS FLOW RATE AT NODE SA

INITIAL GSA

MASS FLON RATE AT MCDE SA

TIME DERIVATIVE OF HSAS

MASS FLOW RATE AT NODE SC

MASS PLOR RATE AT NODE SC

CONTROI ROD POSITION

TIHE DER IVATIVE OF $x$

D-AXIS BLONDEL REACTANCE

QOALITY AT EXHAOST OF TURBINE

QUALITY AT NODE 53

ACTUAL LOCATION, MEASORED FROH CENTERIINE

OF CORE, OF MAXIMOM TEMP AT CIAD OOTEE

SORPACE IN THE AVERAGE CHAMIEL

ACTUAL LOCATION, UEASURED PROM CENTERIINE OF CORE, OF MAXIUOU TEMP AT CIAD OUTEE

SURFACE IN THE HCT CHANNEI

THEORETICAL LOCATION, MEASURED FRCM

CENTERIINE OF CORE, OP MAXINUM TEMP AT

CLAD OUTER SURFACE IN THE HOT CHANNEI

TH EORETICAL LOCATICH, MEASURED FEOM

CENTERLINE OF CORE, OP IAXIMUD TEMP AT

CLAD OUTER SURFBCE IN THE AVERAGE CHANNEL

ACTUAL LOCATION, MEASURED PROH CENTERIINE OF CORE, OP MAXIMCU TEUP AT FUEL

CENTERIINE IN THE AVEEAGE CHANNEL

ACTUAL LOCATION, MEASURED FROA CENTEEIINE

OF CORR, OF TRE HAXIMUD TEUP AT ROEL

CENTERIINE IN THE HOT CHANNEI

THEORETICAL LOCATION, MEASURED FROM

CENTERLINE OF CORE, OF MAXIMUB IEUP AT

CENTERIINE IN THE HOT CHANNEI

THEORETICAI LOCATICN, MEASURED FROM

CENTERLINE OP THE CCRE, CF THE MAX TEMP 


$\begin{array}{ll}\text { YS5 } & \text { FT } \\ \text { YSHAX } & \text { FT } \\ \text { YSMM } & \text { FT } \\ \text { YSMTH } & \text { FT } \\ \text { YSMXTH } & \text { FT }\end{array}$

AT POEL CENTERIINE IN THE AVERAGE CHANNEL HEIGHT OF SATURATED VATER IN STEAL DRUM ACTUAL LOCATICN, MEASURED FROY CENTERLINE OP CORE, OF MAXILUU TEMP AT FOEL SURFACE IN THE ATERAGE CBANNEL

ACTUAL LOCATICN, MEASURED FRCU CELTERIINE OP CORE, OF MAXIMUU TERP AT FUEL SURFACE IN HOT CBANNEI

THEORETICAL LOCAIION, MEASURED FROM CENTERIINE OF CORE, OF UAXIMUU TEMP AT FUEL SURFACE IN THE HOT CHANNEL

THEORETICAL LCCATICN, UEASURED FROM CENTERLINE OF CORE, OP MAXIMOU TEMP AT FUEL SURFACE IN THE AVERAGE CHANNEL 


\section{APPENDIX B}

BRENDA, a DARE $P$ Input File

The following 26 pages is a listing for BRENDA, a DARE $P$ input file containing 1550 lines (card images). The structure of the blocks is described earlier, in the section entitled DARE P. The listing contains the following:

$\begin{array}{ll}\text { Block Name } & \text { Line Numbers } \\ \text { Method } & 00010 \\ \text { Derivative } & 00020-11990 \\ \text { Tables } & 12040-13930 \\ \text { Functions } & 13950-14270 \\ \text { Initial Conditions } & 14320-15200 \\ \text { Output Commands } & 15230-15490\end{array}$

The dollar sign that introduces each of the first four blocks is in column 2. An asterisk denoting a comment is in column 1. Equations begin in column 7 , but may be indented further. A continuation is signified by a dollar sign in column 6 . Several initial conditions separated by commas may appear on a single line. The comment that appears just before each output command is printed as an identifying label in the output, together with the output commands and appropriate column headings. For other details, see the book by Korn and Wait (1978). 


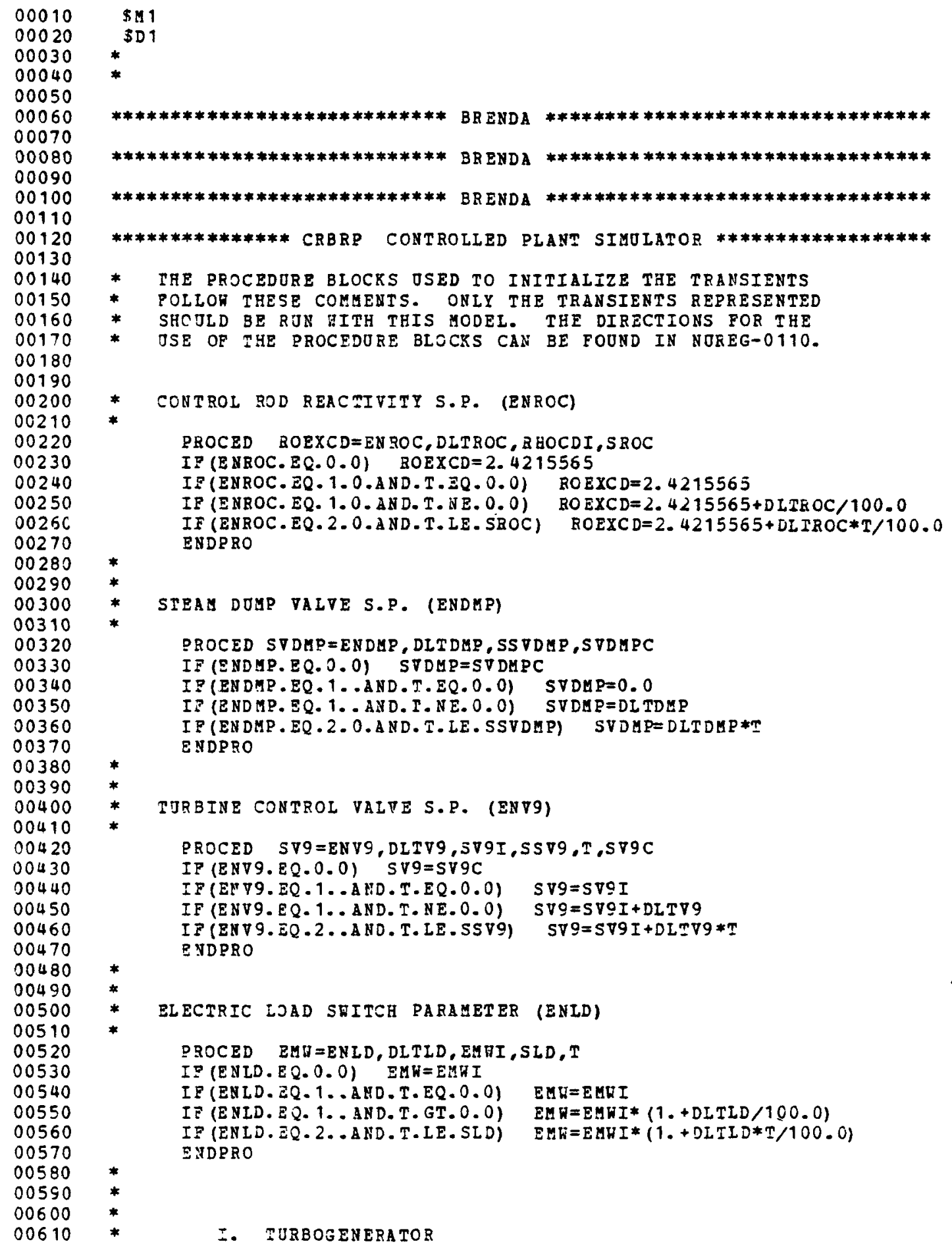




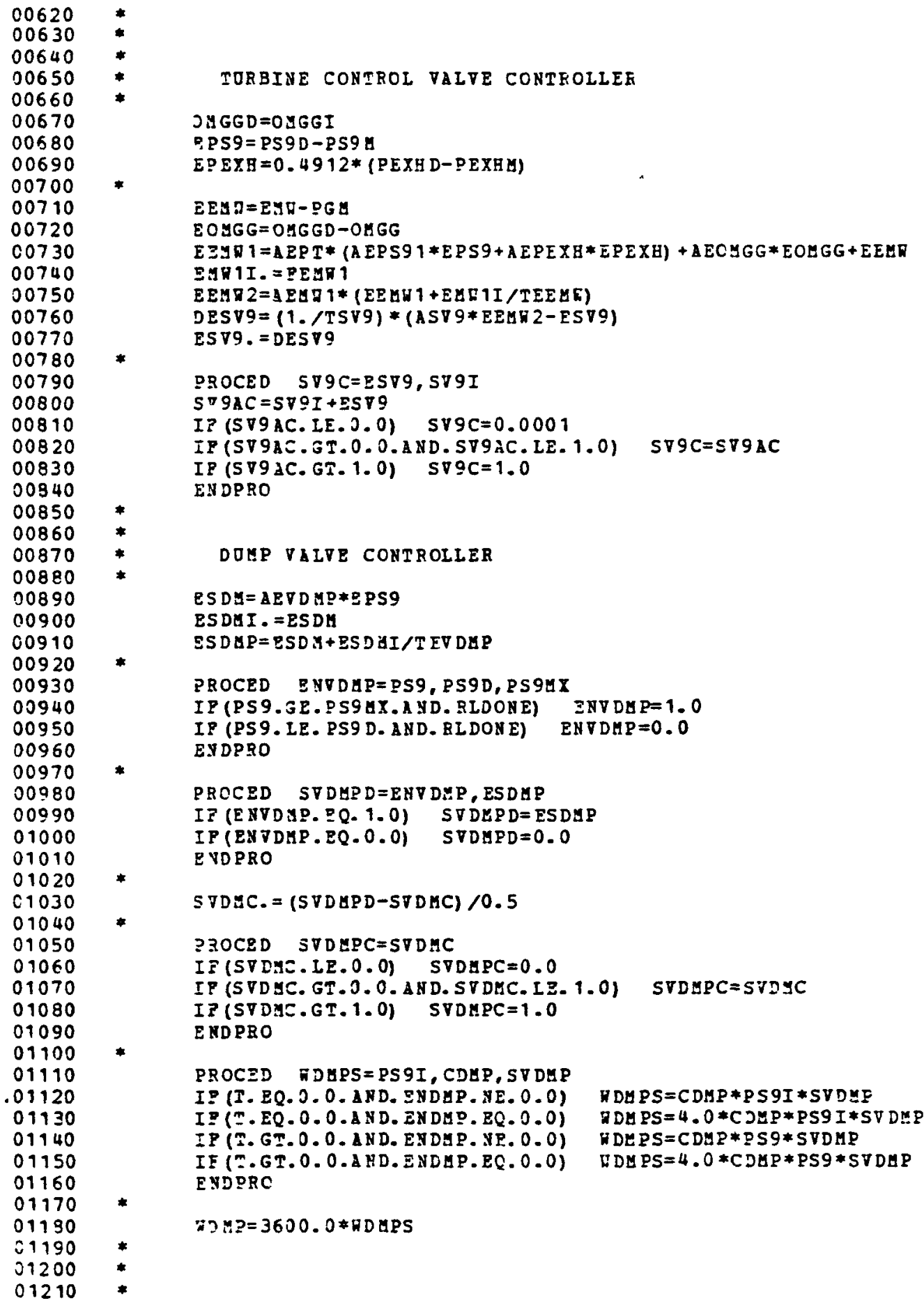




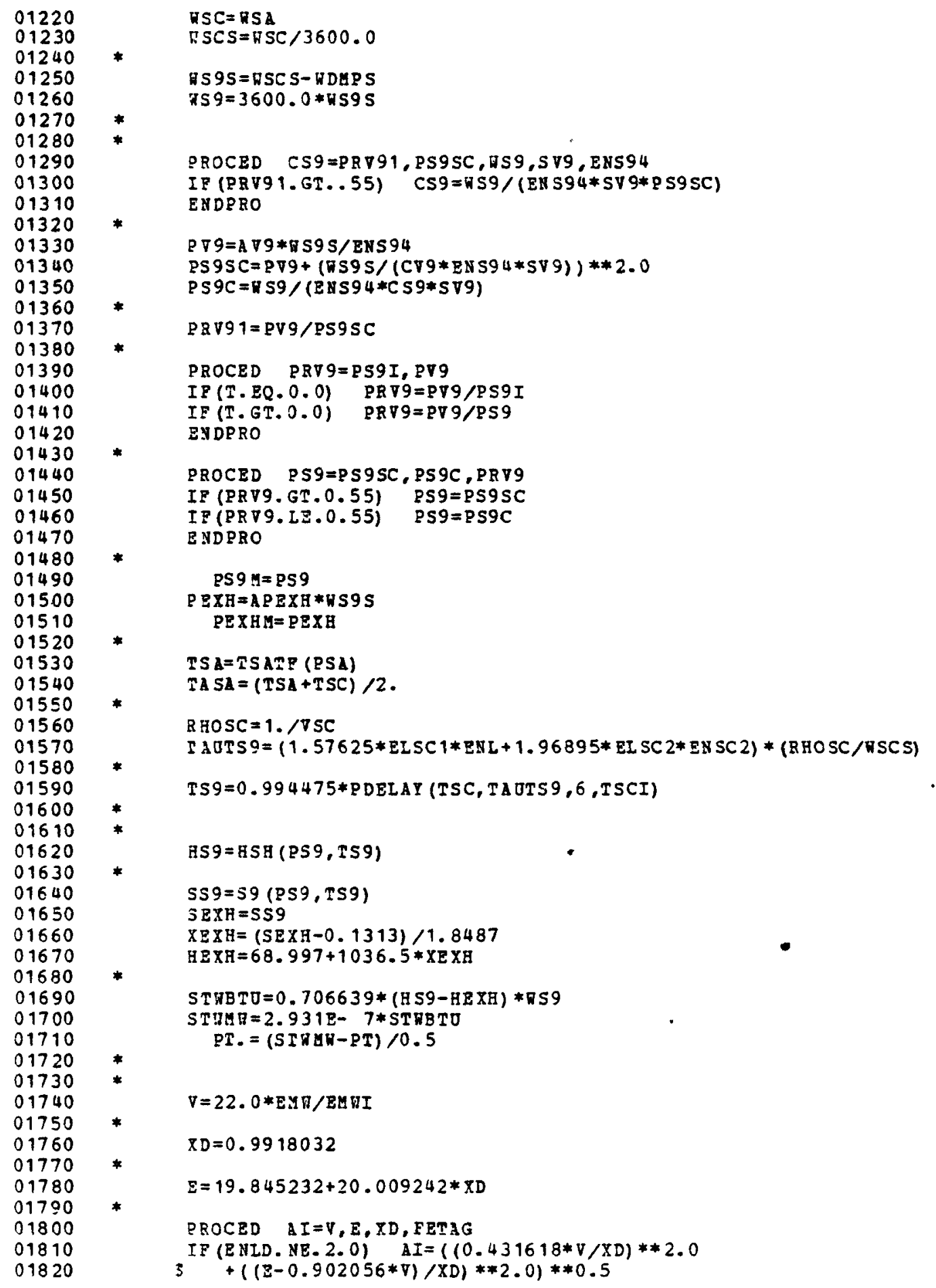




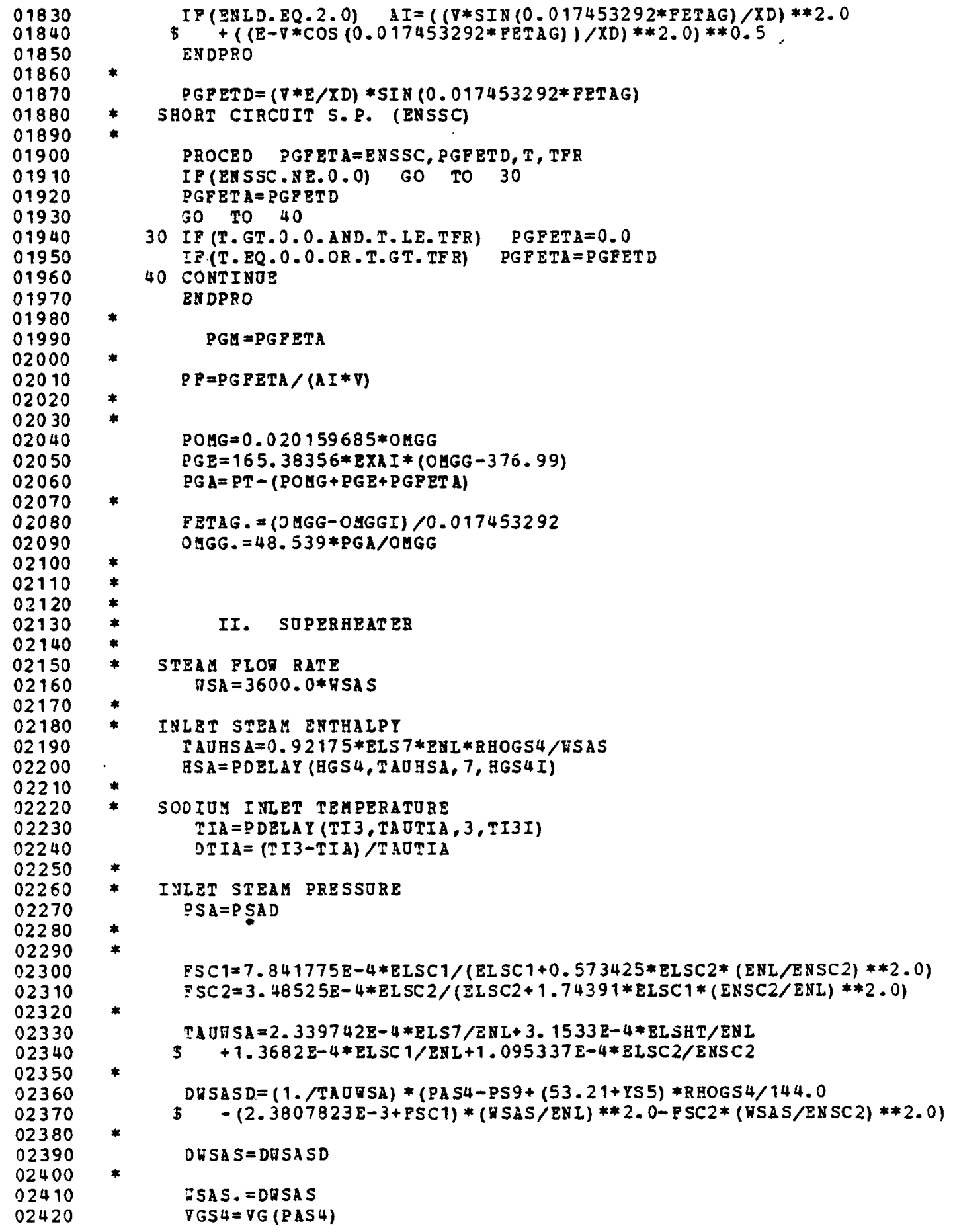




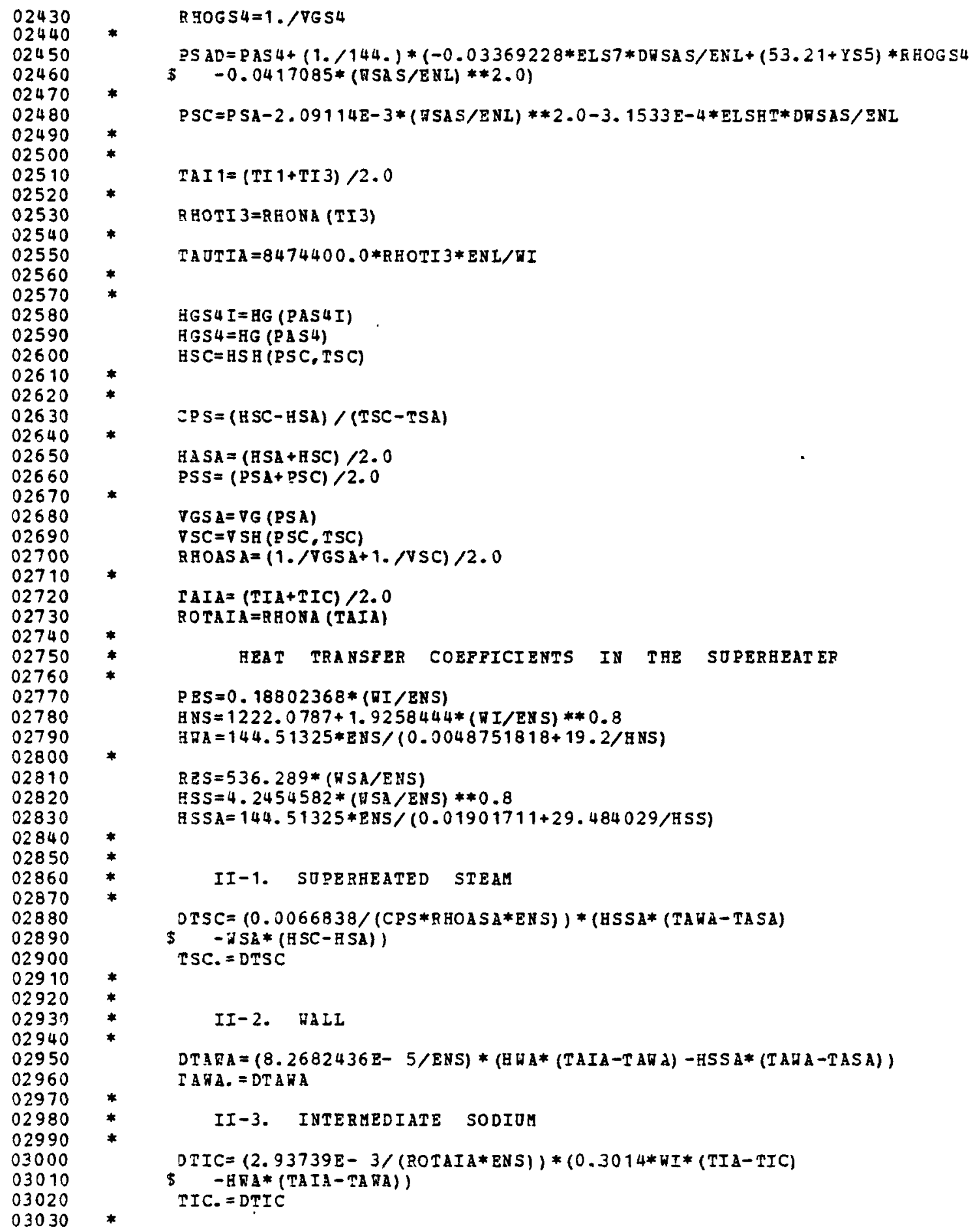




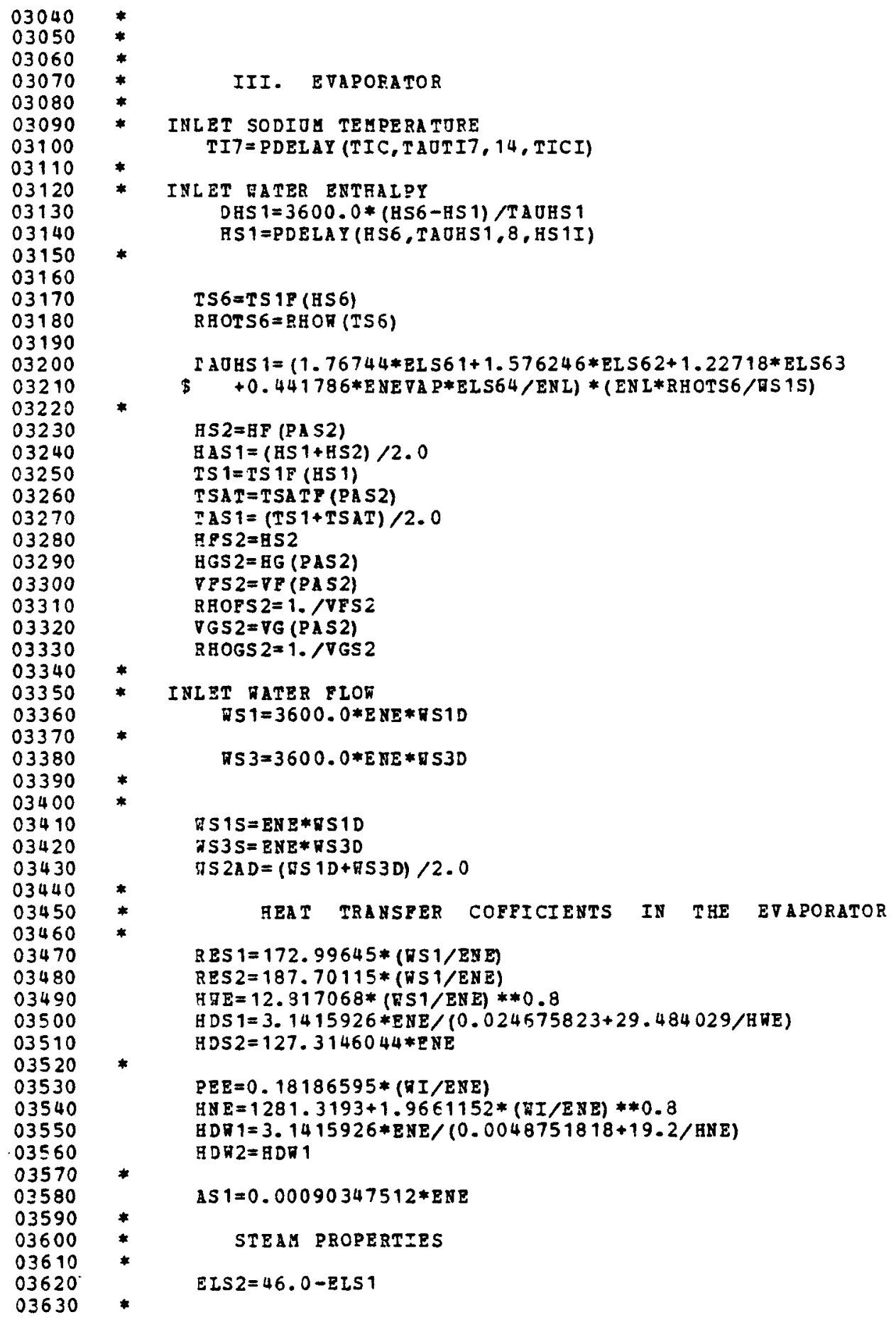




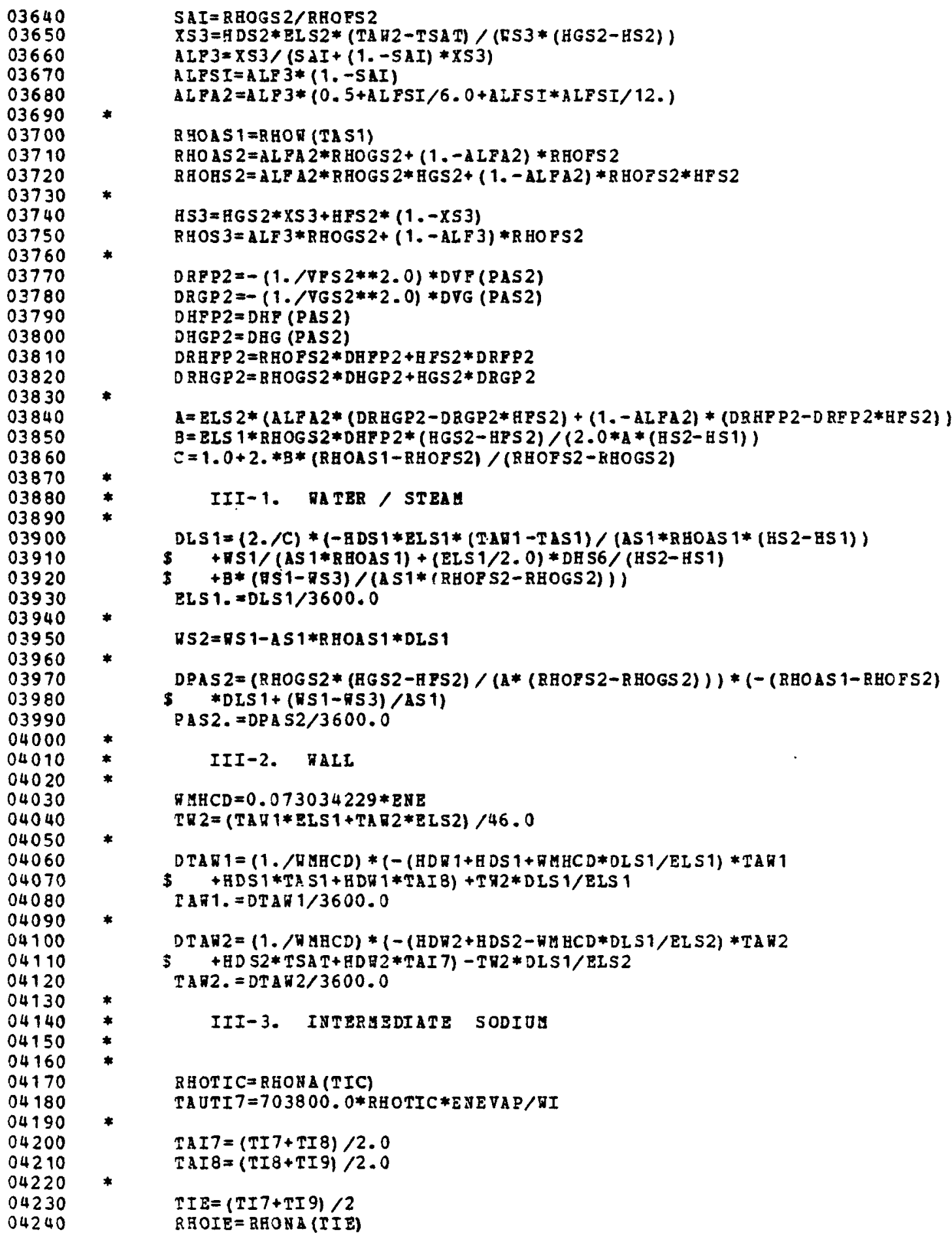




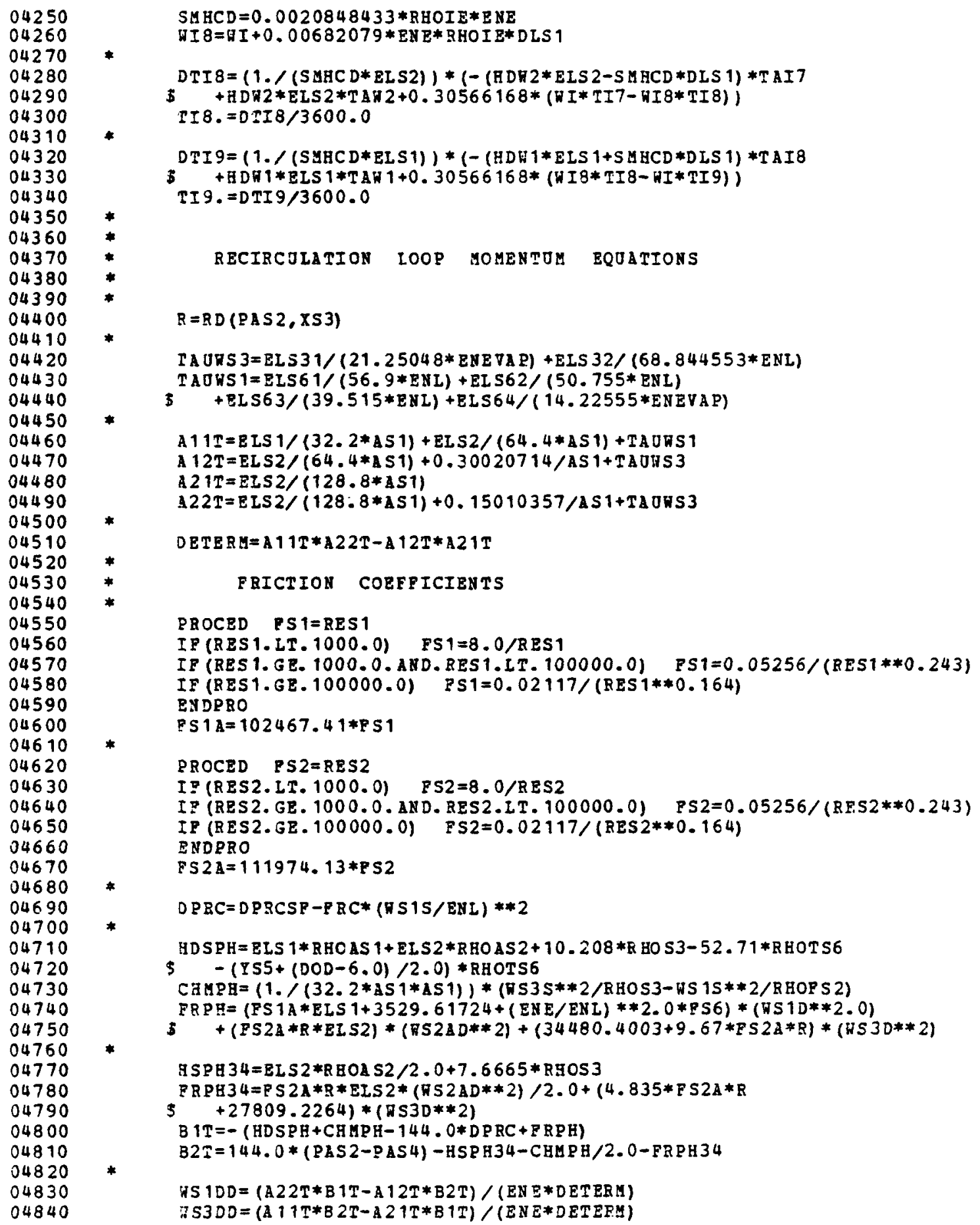




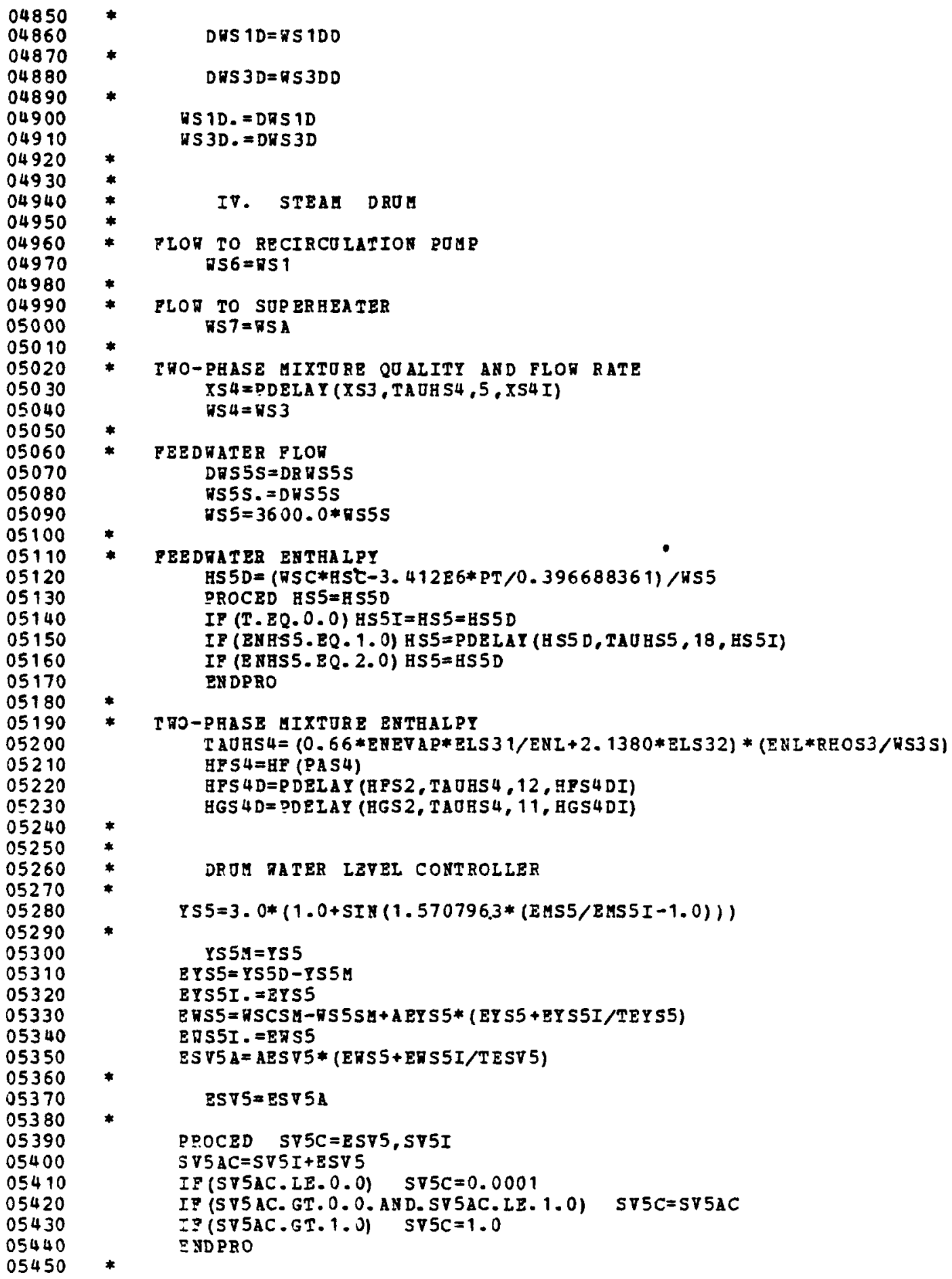




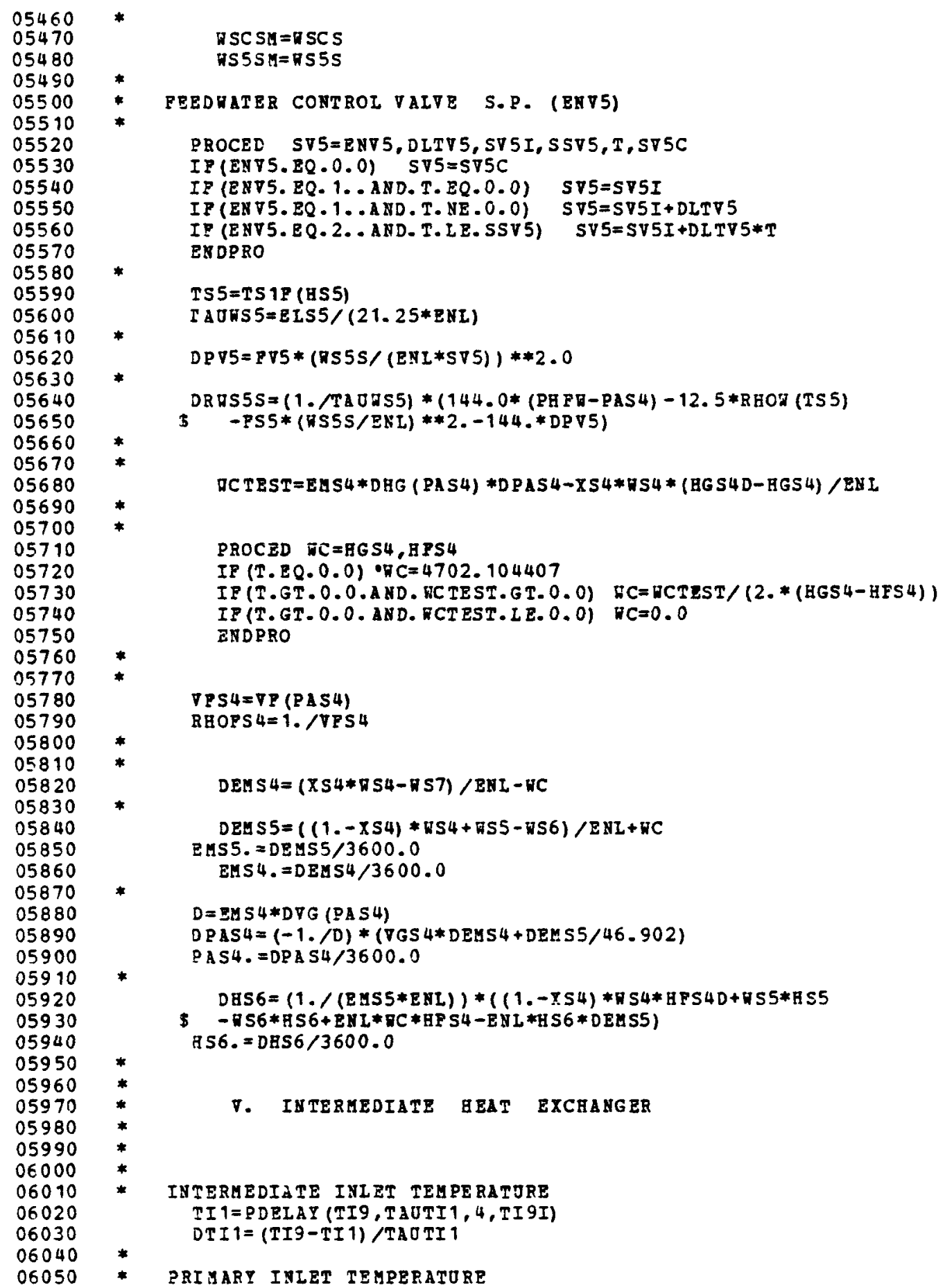




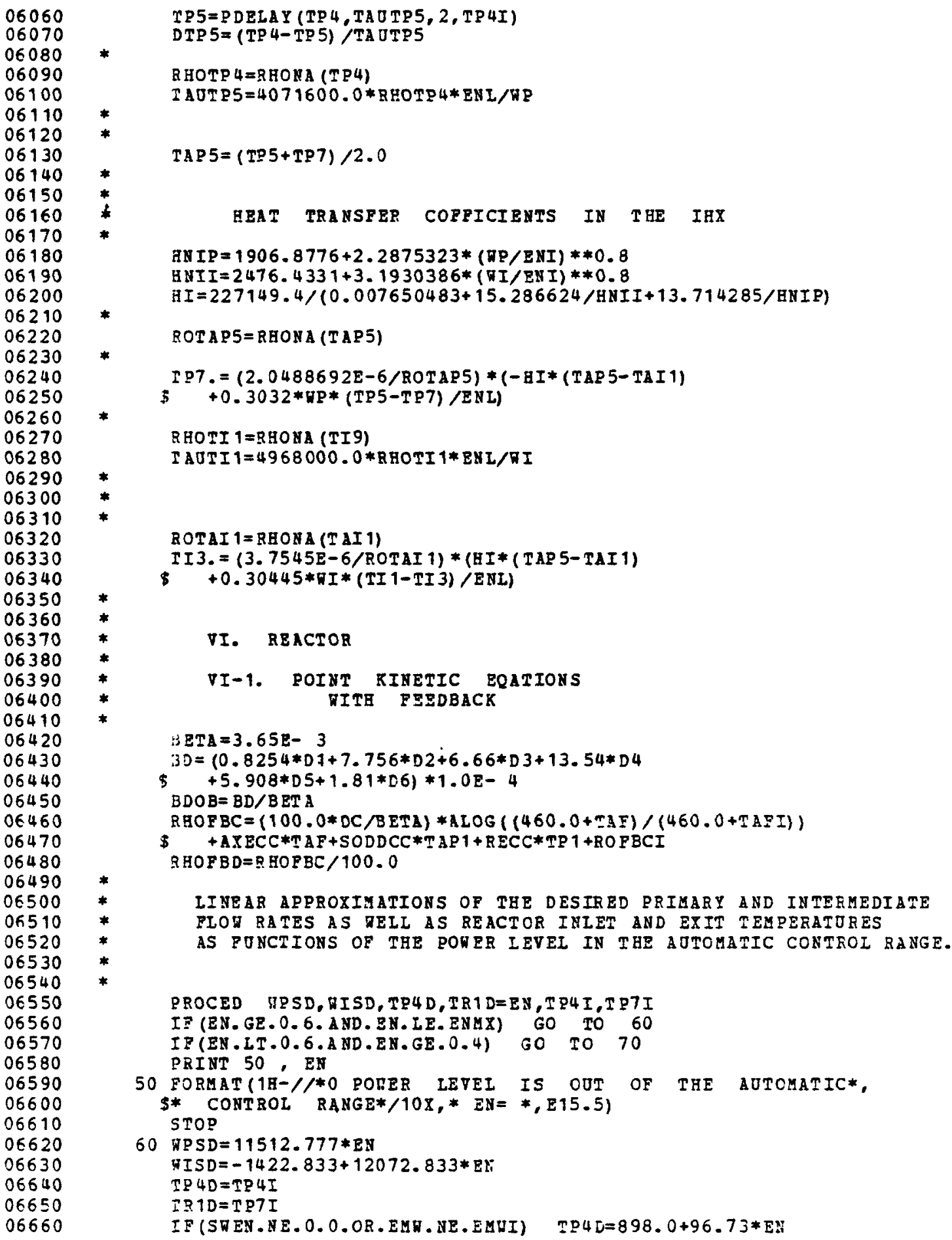




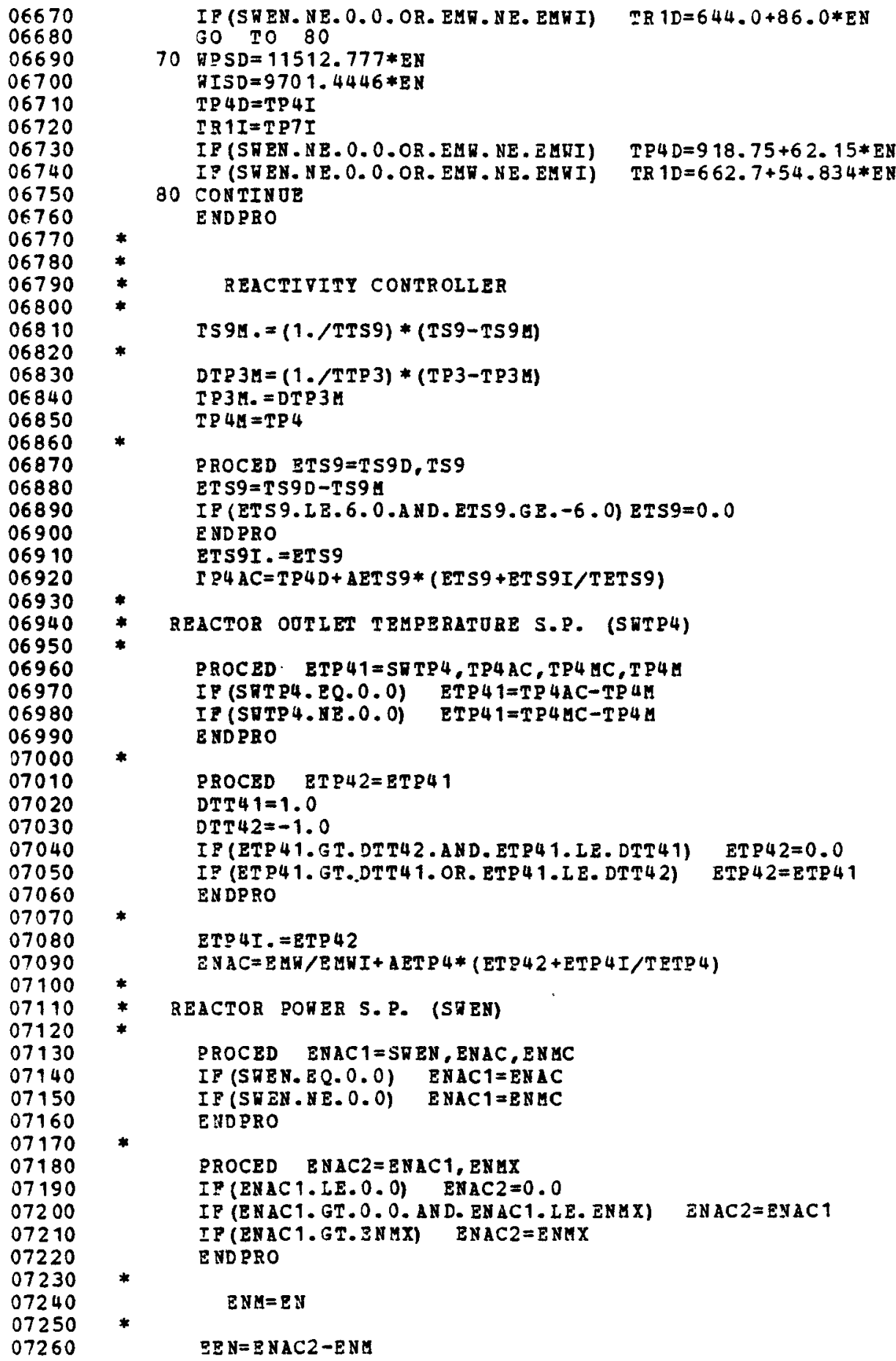


EENI. $=E E R$

$E C R=A B E N *(E E N+E E N I / T E E N)$

07270

07290

07300

07310

07320

07330

07340

07350

07360

07370

07380

07390

07400

07410

07420

07430

- 07440

07450

07460

07470

07480

07490

07500

07510

07520

07530

07540

07550

07560

07570

07580

07590

07600

07610

07620

07630

07640

07650

07660

07670

07680

07690

07700

07710

07720

07730

07740

07750

07760

07770

07780

07790

07800

07810

07820

07830

07840

07850

07860

07870

PROCED $\nabla C R=V C R O U T, V C R I X$

DTVCR $1=0.001$

DT $T C R 2=-0.001$

IF (BCR.GT.DTVCR2 AND.ECR.IT.DTVCR1) $\nabla C K=0.0$

IF (BCR.GT. DTVCR1) $\quad V C R=V C R O U T$

IP (ECR.LT. DTVCR2) VCR =-VCRIN

B NDPRO

$\mathrm{X}_{*}=\mathrm{VCR}$

ROCEXD $=$ ROCRHD*FROCRX (X)

ROCDAC $=$ ROCRXD +ROEXCD

RHOCD $=$ ROCDAC

8. $\mathrm{HOD}=\mathrm{RHOCD}+\mathrm{RHOF} \mathrm{BD}$

PROCED E N=BDOB, RHOD

$E N=B D O B /(1.0-R H O D)$

IF (T.EQ.0.0) EN=1.0

ENDPRO

D 1. $=0.0129 *(E N-D 1)$

$D 2 .=0.0312 *(B N-D 2)$

$D 3 .=0.1330 *(E N-D 3)$

$D 4 .=0.3450 *(E N-D 4)$

$D 5 .=1.4100 *(E N-D 5)$

$D 6 .=3.7500 *(E N-D 6)$

VI-2. REACTOR THERHODYNAAIC EQUATIONS

REACTOR INLET TEMPERATORE

TR 1=PDELA Y (TP 7, TA OTR1, 1, TP7I)

$T P C=(5.19) *.(T A F-32.0)$

$T: K=T F C+273.0$

$\operatorname{AKF}=36.100867 /(T P K+129.2)+5.782936 E-13 * T F K * * 3.0$

$\mathrm{AKF}=57.78 * \mathrm{AKFH}$

ICIIDC $=(5 . / 9) *.(\mathrm{TACL}-32$.

TGAP $=$ TCLIDC +273.0+0.34547*QLPR*EN

HDG $=.0347424 *$ SQRT (TGAP) -0.57059

ADG $=$ BDGH*1761.014

WPD $=F F C * Q P / E N P$

$H C=14961.86+51.921027 * H P D * 0.8$

$H R=9.4247778 * \mathrm{ENP} /(0.125 / \mathrm{AKF}+60.00024 / \mathrm{HDG}+52.173914 / \mathrm{HC}$

$+\quad+0.0052151492)$

HR $1=9.4247778 *$ ENP/(0.125/AKP+60.00024/HDG)

HR $2=9.4247778 * E N P /(52.173914 / \mathrm{HC})$

CPCLA D $=C 2316$ (TACL)

TAP. $=2.520883 E-7 *(E P S * P P G C * E H-H R 1 *(T A F-T A C L))$ 


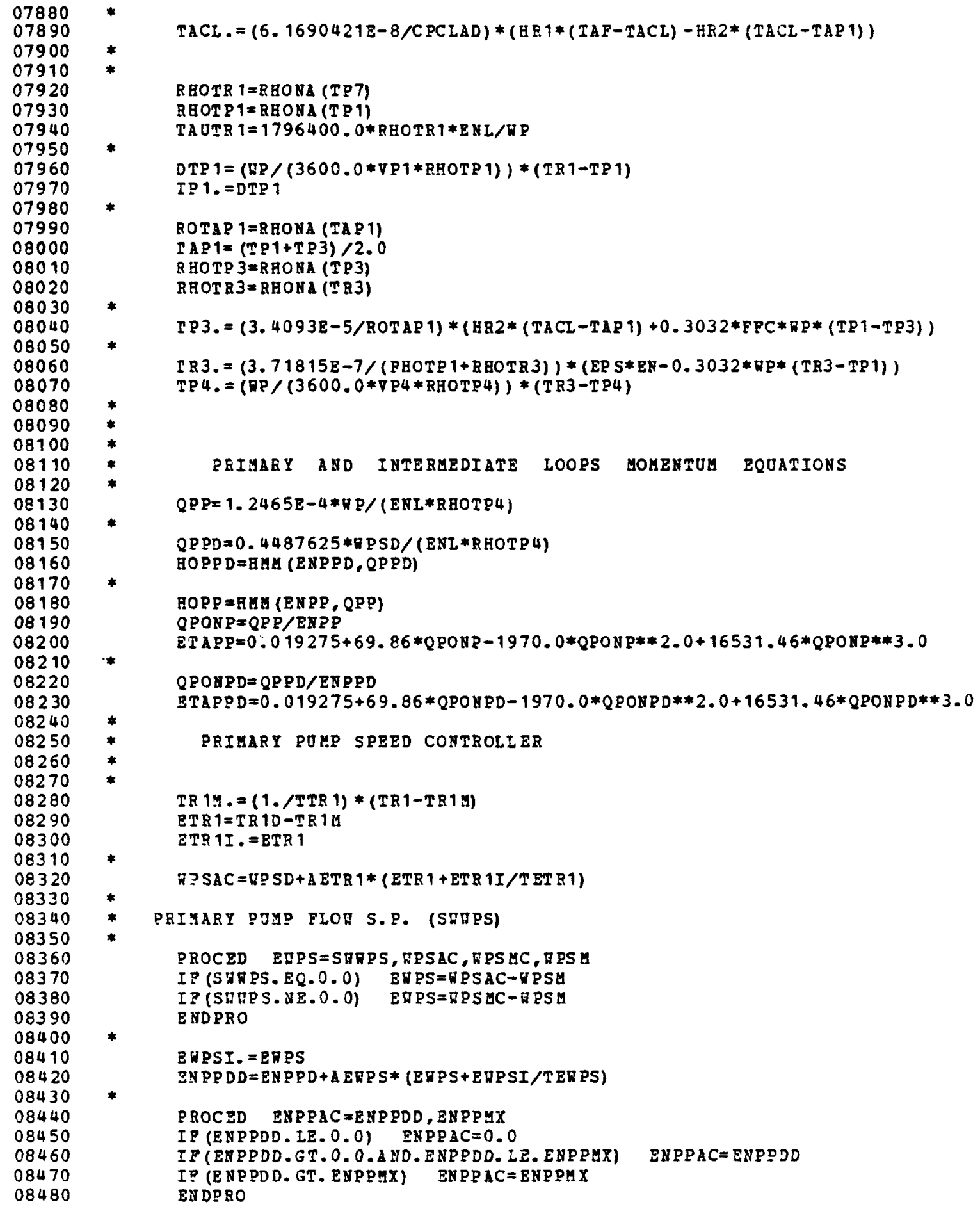




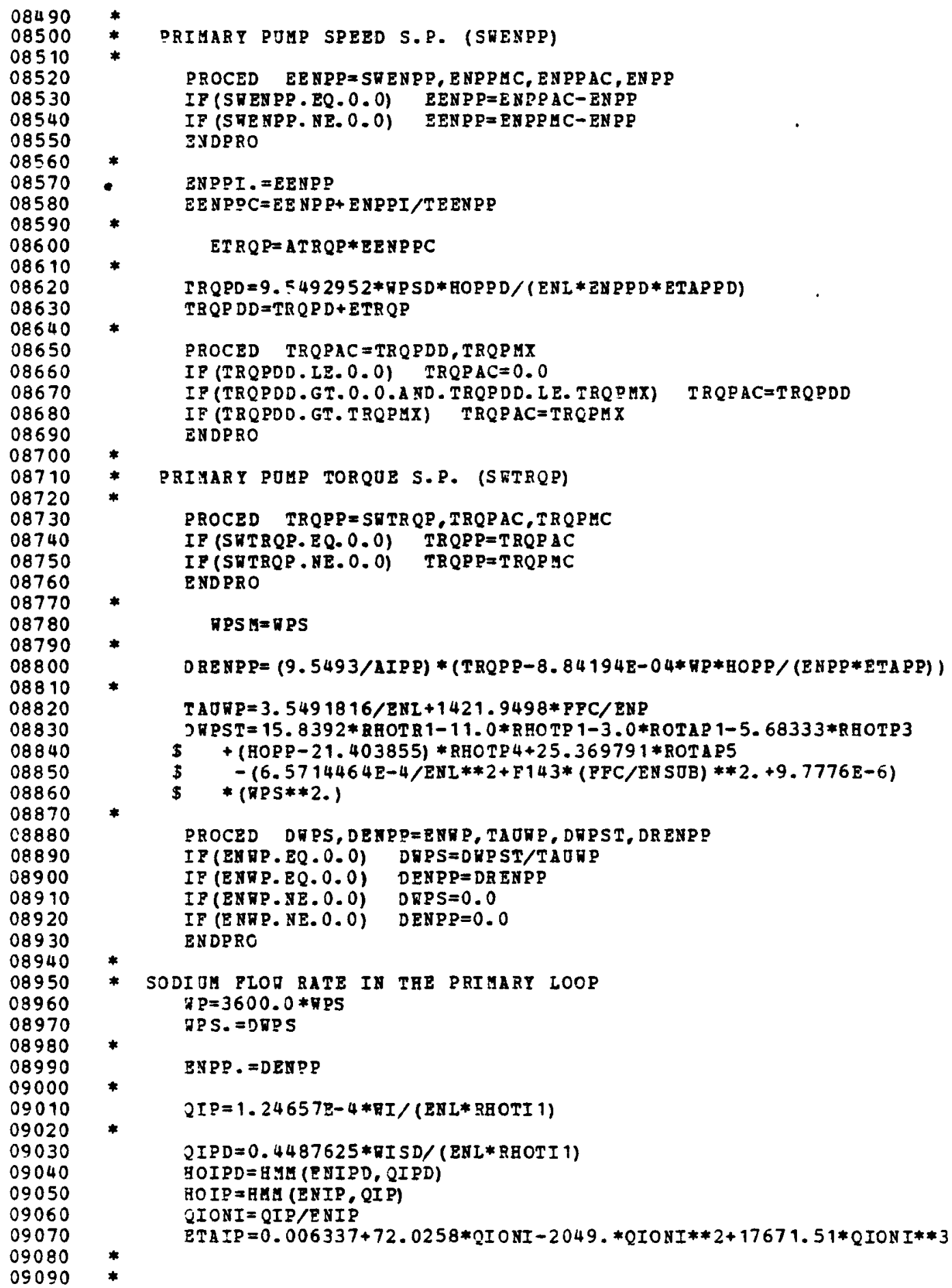

PRIMARY PJMP SPEED S.P. (SWEXPP)

PROCED EENPP $=$ SWENPP, ENPPMC, ENPPAC, ENPP

IF (SNEN PP . EQ.0.0) EENP P=ENPP AC-ENPP

IF (SHENPP. NE. 0.0) EEN P $=$ ENPP IC-ENPP

ZXDPRO

ZNPPI . =EENPP

EENPDC $=E E N P P+E N P P I / T E E N P P$

ET $R Q P=A T R Q P * E E M Q P C$

$T R Q P D=9.5492952 * W P S D * H O P P D /(E N L * E Q P P D * E T A P P D)$

$T B Q P D D=T R Q P D+E T R Q P$

PROCED TROPAC $=$ TROPDD, TROPMX

IP (TRQPDD. LE.0.0) TRQPAC $=0.0$

IP (TRQPDD.GT. 0.0.AND.TRQPDD.LE.TRQPMX) TRQPAC=TRQPDD

IF (TRQPDD.GT. TRQPMX) TRQPAC=TRQPMX

ENDPRO

PRIMARY PDHP TORQUE S.P. (SWTRQP)

PROCED TRQPP=SETRQR, TRQPAC, TRQPEC

IF (SHTRQP. EQ.0.0) TRQRP=TRQP AC

IF (SWTRQP . NE. 0.0) TRQP $=$ TRQP IC

ENDPRO

MPS M $=$ ตPS

$D R E N P P=(9.5493 / A I P P) *(T R Q P Q-8.84194 E-04 * H P * H O P P /(E N P P * E T A P P))$

$T A O N P=3.5491816 / E N L+1421.9498 * F F C / E N P$

J NPST $=15.8392 *$ RROTR $1-11.0 * R$ ROTP 1-3.0*ROTAP 1-5.68333*RHOTP3

$5+$ (HOPP -21.403855 ) * RHOTP $4+25.369791 *$ ROTAPS

$\$ \quad-(6.5714464 \mathrm{E}-4 / \mathrm{ENL} * * 2+\mathrm{F} 143 *(\mathrm{FFC} / \mathrm{ENSOB}) * * 2 .+9.7776 \mathrm{E}-6)$

$\$ \quad *($ RPS**2.)

PROCED DNPS, DENPP=ENWP, TAONP, DNPST, DRENPD

IF (ENAP.EQ.0.0) DNPS=DKPST/TAONP

IF (ENTP.EQ.0.0) DENPP=DRENPP

IP (ENTP.NE.0.0) DEPS $=0.0$

IF (ENRP.NE.0.0) DENPP=0.0

ENDPRO

* SOdIJl pLOT RATE IN THE PRIMART LOOP

$A P=3600.0 * \nabla P S$

TPS. $=$ DNPS

BNPP. = DENPP

$2 I P=1.24657 \mathrm{E}-4 * \pi I /($ ENL*RHOTI 1$)$

QIPD $=0.4487625 * \pi I S D /(E N L *$ RHOTI 1$)$

HOIPD $=$ GIM (ENIPD, QIPD)

HOIP $=\mathrm{HHA}$ (ENIP, QIP)

QIONI $=Q I P / E N I P$

ETAIP $=0.006337+72.0258 * 2 I$ ONI $-2049 . * Q I O N I * * 2+17671.51 * Q I O N I * * 3$ 


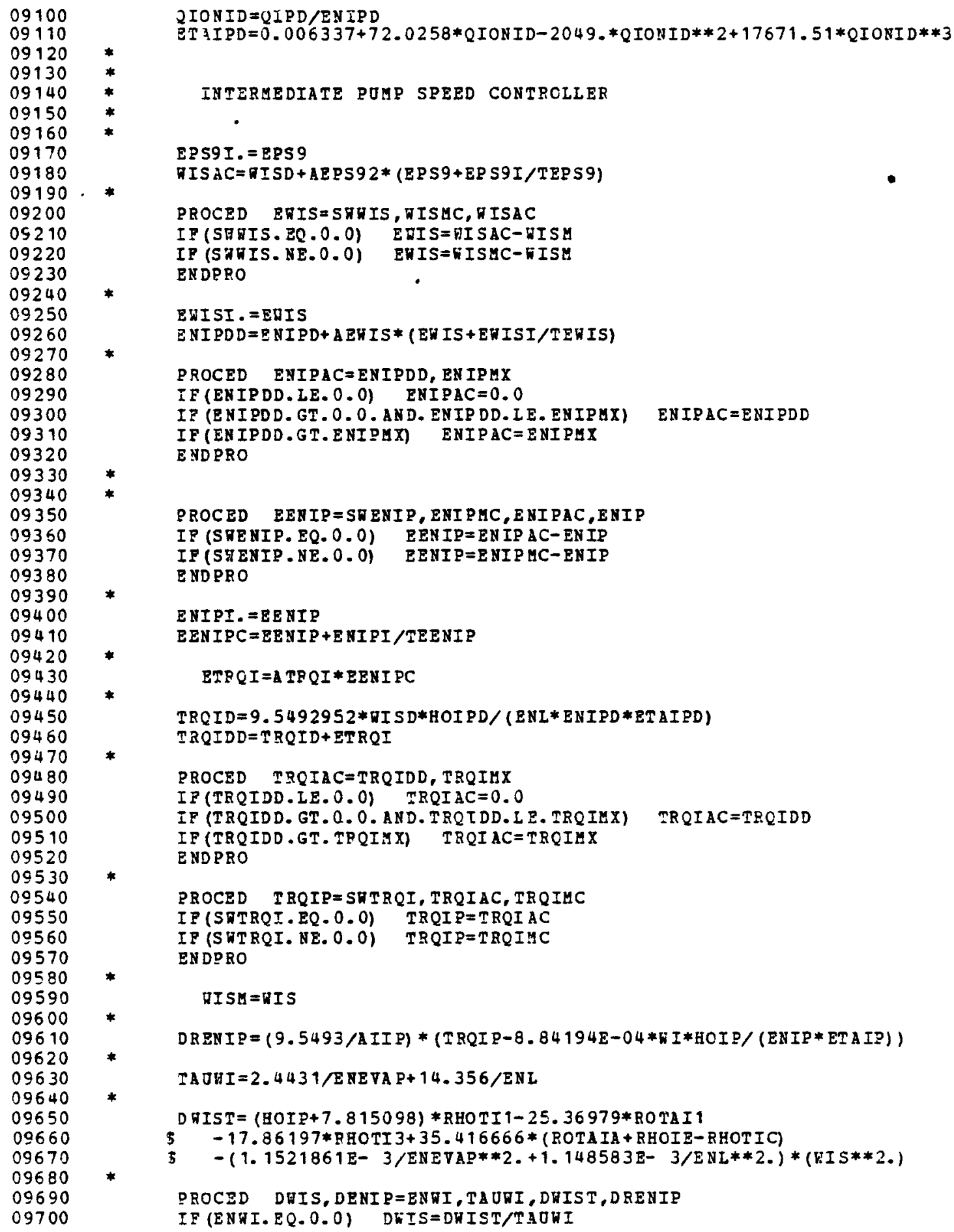




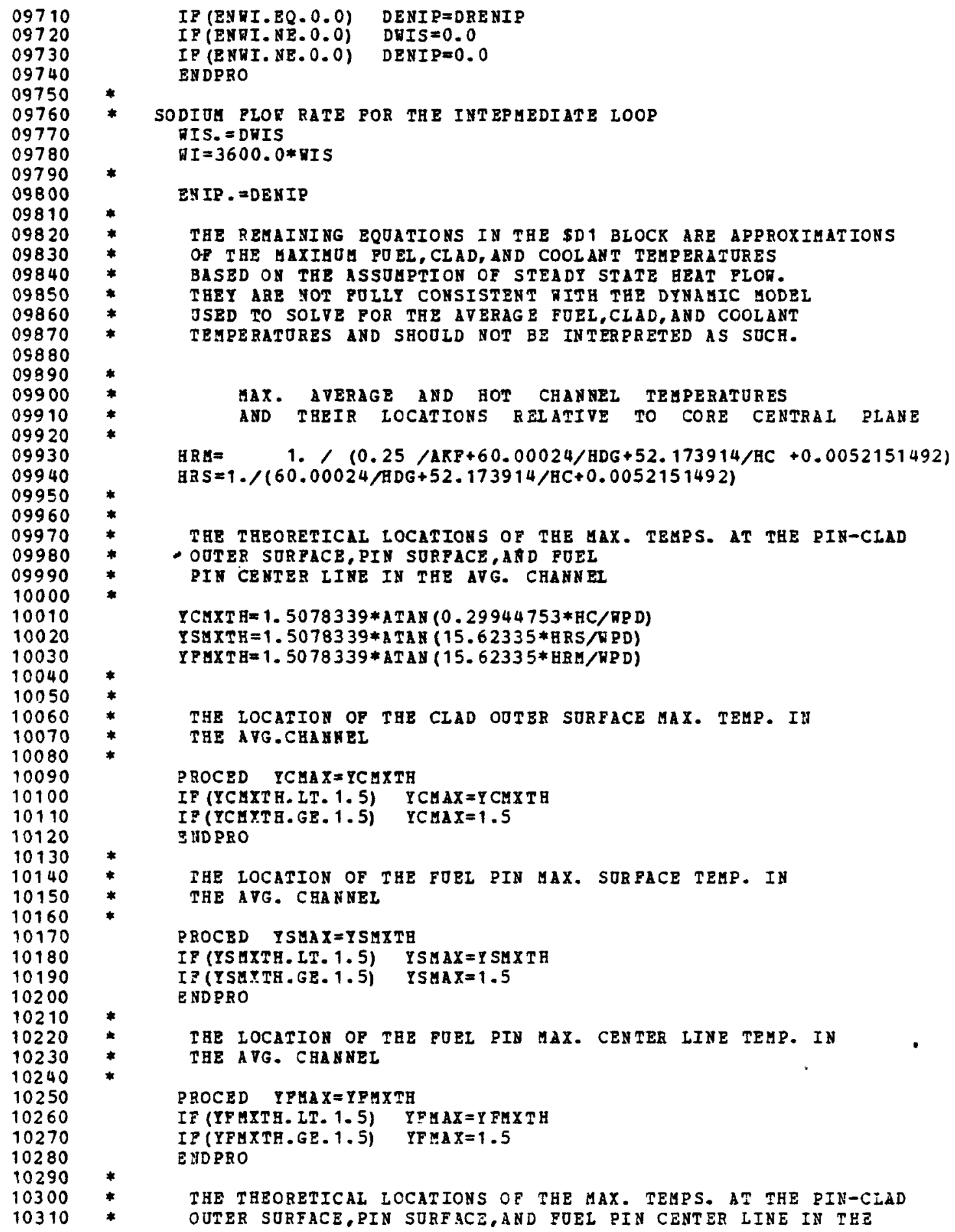




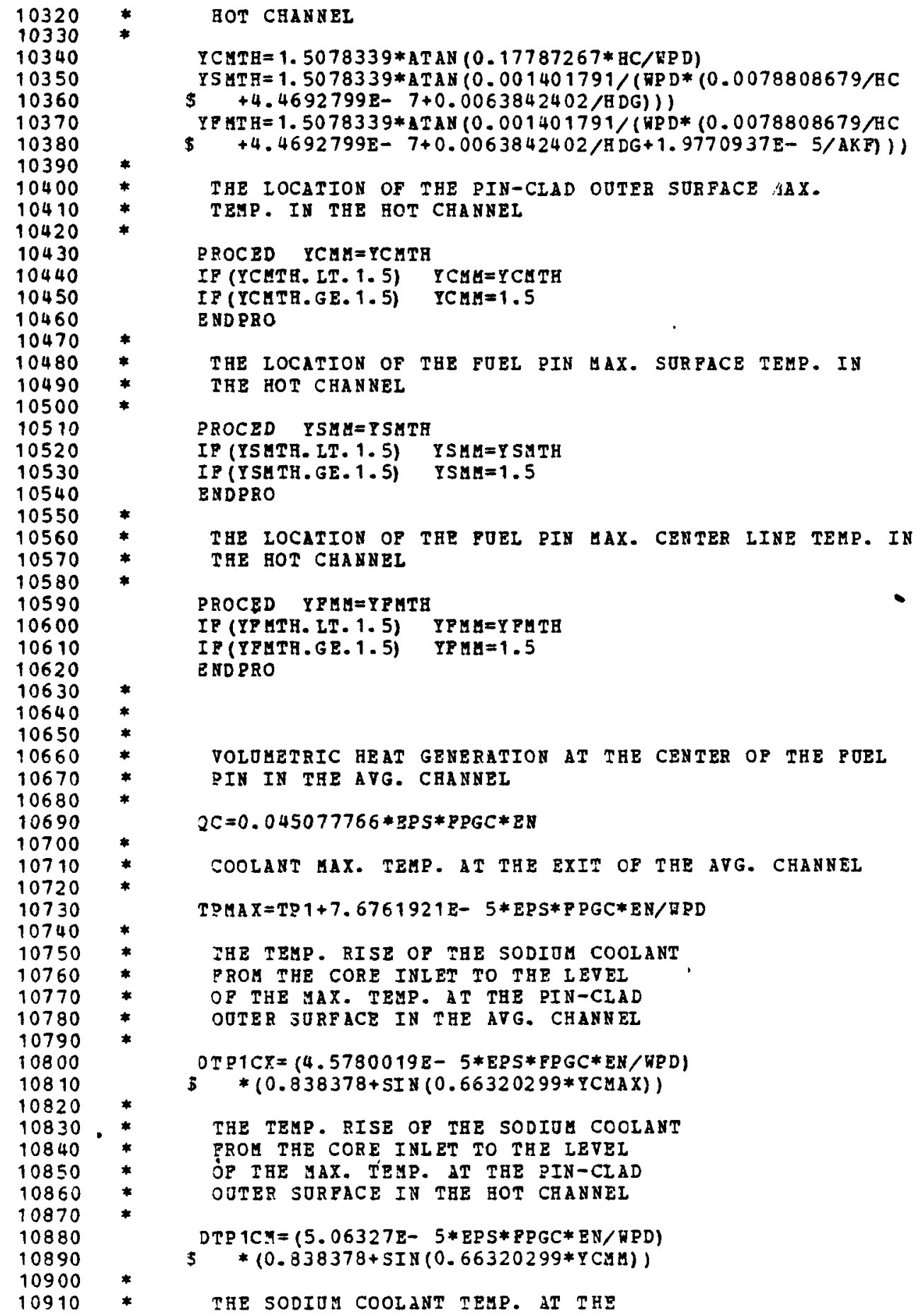


LEVEL OF THE MAX. TEMP. OF THE

PIN-CLAD OUTER SURFACE IN THE AVG. CHANNEL

TP YCA $X=$ IP $1+$ DTP TCX

THE DIPFERENCE BETEEEN THE PIN-CIAD

OUTER SORPACE TEMP. AND TBE

SODIUE COOLANT TEHP. AT THE LETEL

OF THE MAX. TEUP. OF THE CLAD

OOTER SORFACE IN THE AVG. CHANNEI

DTPCAX $=0.0033915082 * Q C * \operatorname{COS}(0.66320299 *$ ICMAX) $/ \mathrm{HC}$

THE DIPPERENCE BETWEEN THE PIN-CLAD

OOTER SORFACE TEMP. AND THE

SODIOH COOLANT TEMP. AT THE LEVEL

OP THE HAX. TEUP. OF THE CLAD

OUTER SURPACE IN THE HOT CEANMEL

DTPCM $=0.0033915082 * Q C * \operatorname{COS}(0.66320299 *$ YCMM $) / H C$

MAX. PIN-CLAD OUTER SUREACE TEMP. IN THE ZVG. CBANEL

$\operatorname{TCHAX}=\operatorname{TPTCMX}+$ DTPCHX

THE TEMP. RISE OF TRE SODIOA COOLANT

PROM THE COBE TNLET TO THE LEVEL

OF THE HAX. TEMP. AT THE FUEL

PIN SURFACE IN THE AVG. CHANNEL

OTP1SX $=(4.5780019 E-5 * E P S * P P G C * E N /$ RPD $)$

$\$ *(0.838378+\operatorname{SI} \&(0.66320299 * I S \Delta A X))$

THE SODIOU COOLANT TEMP. AT THE LEVEL

OP THE MAX. TEMP. OP THE

POEL PIY SURFACE IN THE HOT CHANNEL

DIP1S $U=(5.06327 E-5 * E P S * F P G C * E N / H P D)$

\$ $*(0.838378+$ SIN $(0.66320299 *$ YSMU) $)$

THE SODIOM COOLANT TEMP. AT THE LEVEL

OP THE HAX. TEUP. CF THE

POEL PIN SURFACE IN THE AVG. CHANNEL

T PIS MX $=$ TP $1+$ DTP $15 X$

MAX. FUEI PIN SUREACE TEMP. IN TEE ATG. CHANHEL

ISMAX $=$ TPYSAX $+6.5003905 \mathrm{E}-5 * Q \mathrm{Q} * \operatorname{COS}(0.66320299 * Y S M A X) / \mathrm{HRS}$

TRE TEAP. RISE OF THE SODIOM COOLANT

PROM THE CORE INIET TO TME IETEL

OF THE GAX. TEMP, AT THE FOEL PIN

CENTER IINE IN THE AVG. CHANNEL

DTP1FX $=(4.5780019 \mathrm{E}-5 * \mathrm{EPS} * \mathrm{FPGC} * \mathrm{EN} / \mathrm{WPD})$

$\$ \quad *(0.838378+\operatorname{SIN}(0.66320299 *$ FMAX) $)$

THE TEYP. RISE OF TFE SODIUY COOLANT

PROM THE CORE INLET TO THE LEVEL

OP THE MAX. TEMP. AT THE FOEL RIN 
11530

11540

11550

11560

11570

11580

11590

11600

11610

11620

11630

11640

11650

11660

11670

11680

11690

11700

11710

11720

11730

11740

11750

11760

11770

11780

11790

11800

11810

11820

11830

11840

11850

11860

11870

11880

11890

11900

11910

11920

11930

11940

11950

11960

11970

11980

11990

12000

12010

12020

12030

12040

12050

12060

12070

12080

12090

12100

12110

12120

12130

CENTER LINE IN THE HOT CHANNEL

DTP $1 F U=(5.06327 E-5 * E P S * F P G C E N / W P D)$

$\$ \quad *(0.838378+$ SIN $(0.66320299 *$ YFMU) $)$

* the sodiua coolant teup. at the level of the max. temp. of the

* poej pin at its center line in the avg. channel

*

TPYFMX=TP1+DTP 1FX

MAX. FUEI PIN TEHP. AT ITS CENTER IINE IN THE AVG. CHANHEL

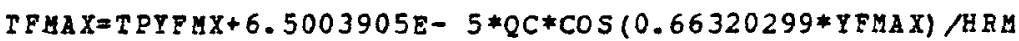

COOLANT MaX. TEMP. IN THE HOT CHANNEL

$T P M M=1.248 *(T P 1+8.4898684 E-5 * E P S * P P G C * E N / R P D)$

PIN-CLAD HAX. OUTER SUREACE TEMF. IN THE HOT CHANNEL

TCMM $=1.248 *(T P 1+D T P 1 C M)+2.323706 * D T P C M$

*

*

*

$$
\$
$$

FOEL PIN MAX. SURPACE TEMP. IN THE HOT CHANNEL

TSAM $=1.248 *($ TP 1 DTP1SH $+(0.0078808679 /$ HC $+4.4692799 \mathrm{E}-7$ $+0.0063842402 / \mathrm{HDG}) * Q C * \operatorname{COS}(0.66320299 * Y \mathrm{SH})$

$*$

PUEL PIA GAX. CENTER LINE TEMP. In IHE hOT CHANNEL

TPMM $=1.248 *(T P 1+D T P 1 P M)+(0.0078808679 / H C+4.4692799 E-7$

$\$ \quad 0.0063842402 / \mathrm{BDG}+1.9770937 \mathrm{E}-5 / \mathrm{AKF}) * \mathrm{QC} * \operatorname{COS}(0.66320299 * \mathrm{YFMG})$

STORE, WS9,PS9, TS9, HS 9,5S9, XEXH, HEXH, STWBTJ, PT, PGFETA, POMG, PGE, \$ EMW, PGA, FET AG, OMGG, AI, E, PF, XD, WSA, \#SC, RHOA SA, CPS, PSA, PSC, TSA, $\$$ TSC, HSA, KSC, TIC, TASA, TA WA, TAIA, ELS1, ELS2, MS 1, MS 3, XS3, ALF3, HS3, \$AW1, TAH2,TAI7, TAI8, TI9, PAS2, ALFA2, HS1, TS1, TAS1, ISAT, B1T,B2T, 5 DETERM, EMS4, EMS5, YS5, PAS4, HS6, RHCD, EN, TAF, TAP1, TAP5, IAI1, TI3,

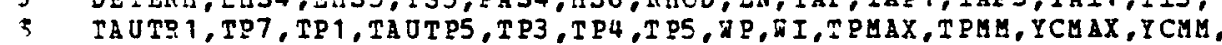

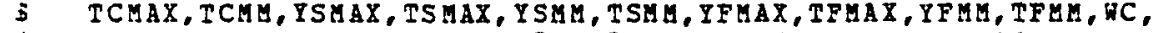
\$ DHPS,DHIS, TAUNP, TAUWI, WS5,HS5,QPP, HOPR, QIP, HOIP,TR T, DNSAS, 3 ROEXCD, X,XCR, TAUTI1, TAOTIA, TAUTI7, TAOHS1, TAUHS4, TAUHSA, TAUTS9, $\$$ TDMP,TI7, TI8, TIA, TI I,TR3, ENPQ, ETAPP, ENIP, ETAIP, DPAS4, DEMS4, 5 DEMS5, TACL, ENAC2, EENI, ECR, EEN

*

$+$

*

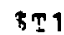

RHONa, 6

* NEXT IS 392.0

572.0

752.0

932.0

1112.0

1292.0
THE INDEPENDENT VARIABLE FOLLONED BY THE DEPENDENT .56 .4

.55 .1

.53 .6

.52 .1

.50 .5

.48 .9 


\begin{tabular}{|c|c|c|}
\hline & $\begin{array}{r}\text { TS1F } 12 \\
374.97 \\
396.77 \\
418.9 \\
430.1 \\
441.4 \\
464.4 \\
487.8 \\
511.9 \\
536.6 \\
562.2 \\
588.9 \\
610.0\end{array}$ & $\begin{array}{l}.400 .0 \\
.420 .0 \\
.440 .0 \\
.450 .0 \\
.460 .0 \\
.480 .0 \\
500.0 \\
.520 .0 \\
540.0 \\
.560 .0 \\
580.0 \\
.600 .0\end{array}$ \\
\hline$\$$ I 3 & $\begin{array}{c}\text { RHOR } 12 \\
400.0 \\
420.0 \\
440.0 \\
450.0 \\
460.0 \\
480.0 \\
500.0 \\
520.0 \\
540.0 \\
560.0 \\
580.0 \\
600.0\end{array}$ & $\begin{array}{l}.53 .648 \\
.52 .798 \\
51.921 \\
51.546 \\
51.02 \\
.50 .0 \\
49.02 \\
47.847 \\
.46 .512 \\
45.249 \\
43.860 \\
.43 .373\end{array}$ \\
\hline$\$ T 4$ & $\begin{array}{l}\text { VF } 6 \\
1200.0 \\
1300.0 \\
1400.0 \\
1500.0 \\
2000.0 \\
2500.0\end{array}$ & $\begin{array}{r}0.0223 \\
.0 .0227 \\
0.0231 \\
.0 .0235 \\
0.00257 \\
0.0287\end{array}$ \\
\hline $5 T 5$ & $\begin{array}{l}\mathrm{HE}, 6 \\
1200.0 \\
1300.0 \\
1400.0 \\
1500.0 \\
2000.0 \\
2500.0\end{array}$ & $\begin{array}{r}.571 .7 \\
.585 .4 \\
.598 .7 \\
.611 .6 \\
.671 .7 \\
.730 .6\end{array}$ \\
\hline$\$ \mathrm{~T} 6$ & $\begin{array}{r}\text { TSATF }, 6 \\
1200.0 \\
1300.0 \\
1400.0 \\
1500.0 \\
2000.0 \\
2500.0\end{array}$ & $\begin{array}{l}.567 .22 \\
.577 .46 \\
.587 .1 \\
.596 .23 \\
.635 .82 \\
.668 .13\end{array}$ \\
\hline$\$ \mathrm{r} 7$ & $\begin{array}{c}\nabla G \quad 6 \\
1200.0 \\
1300.0 \\
1400.0 \\
1500.0 \\
2000.0 \\
2500.0\end{array}$ & $\begin{array}{r}0.3619 \\
.0 .3293 \\
.0 .3012 \\
.0 .2765 \\
.0 .1878 \\
0.1307\end{array}$ \\
\hline
\end{tabular}

ST8 $\exists G, 6$ 


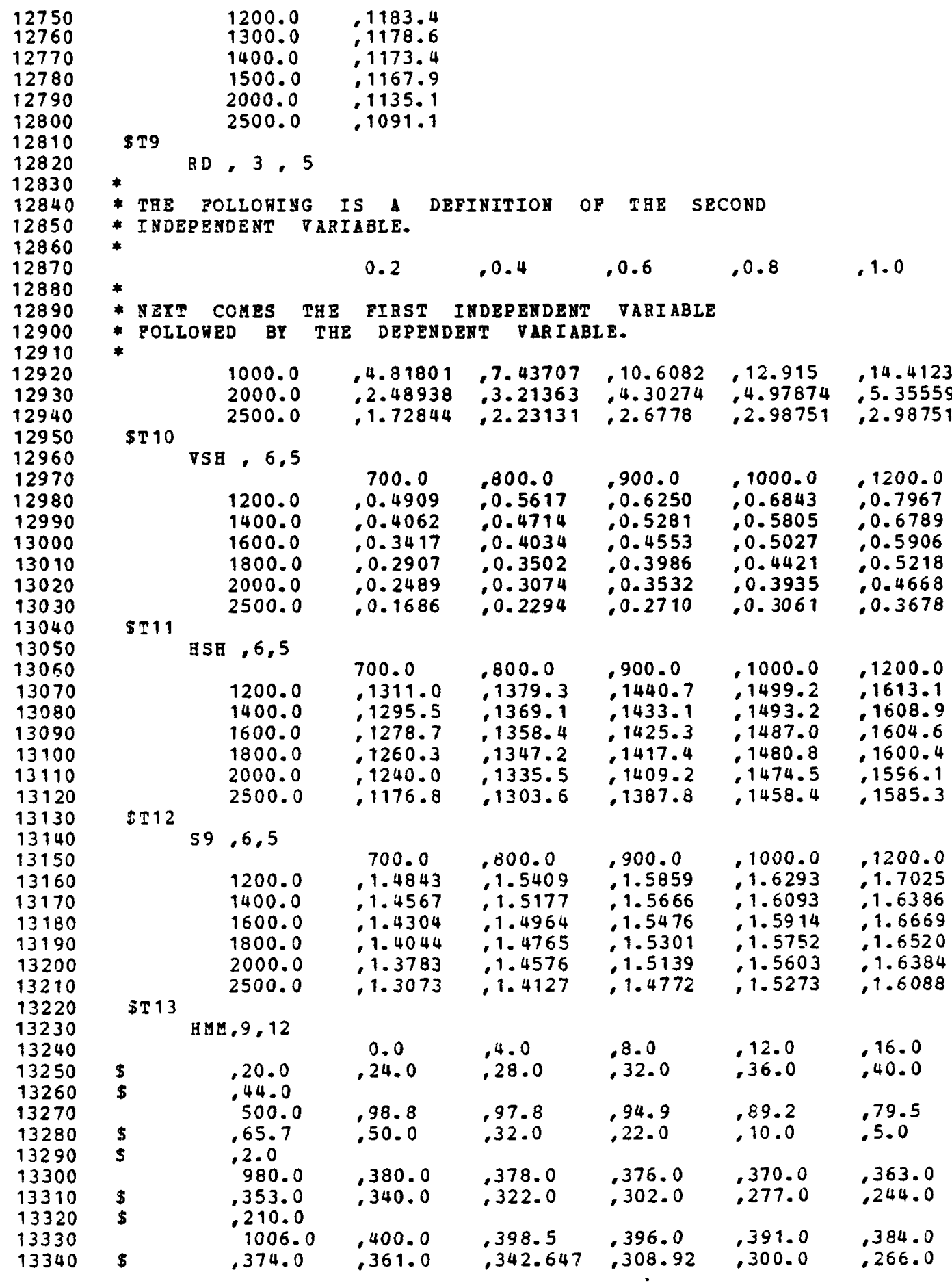




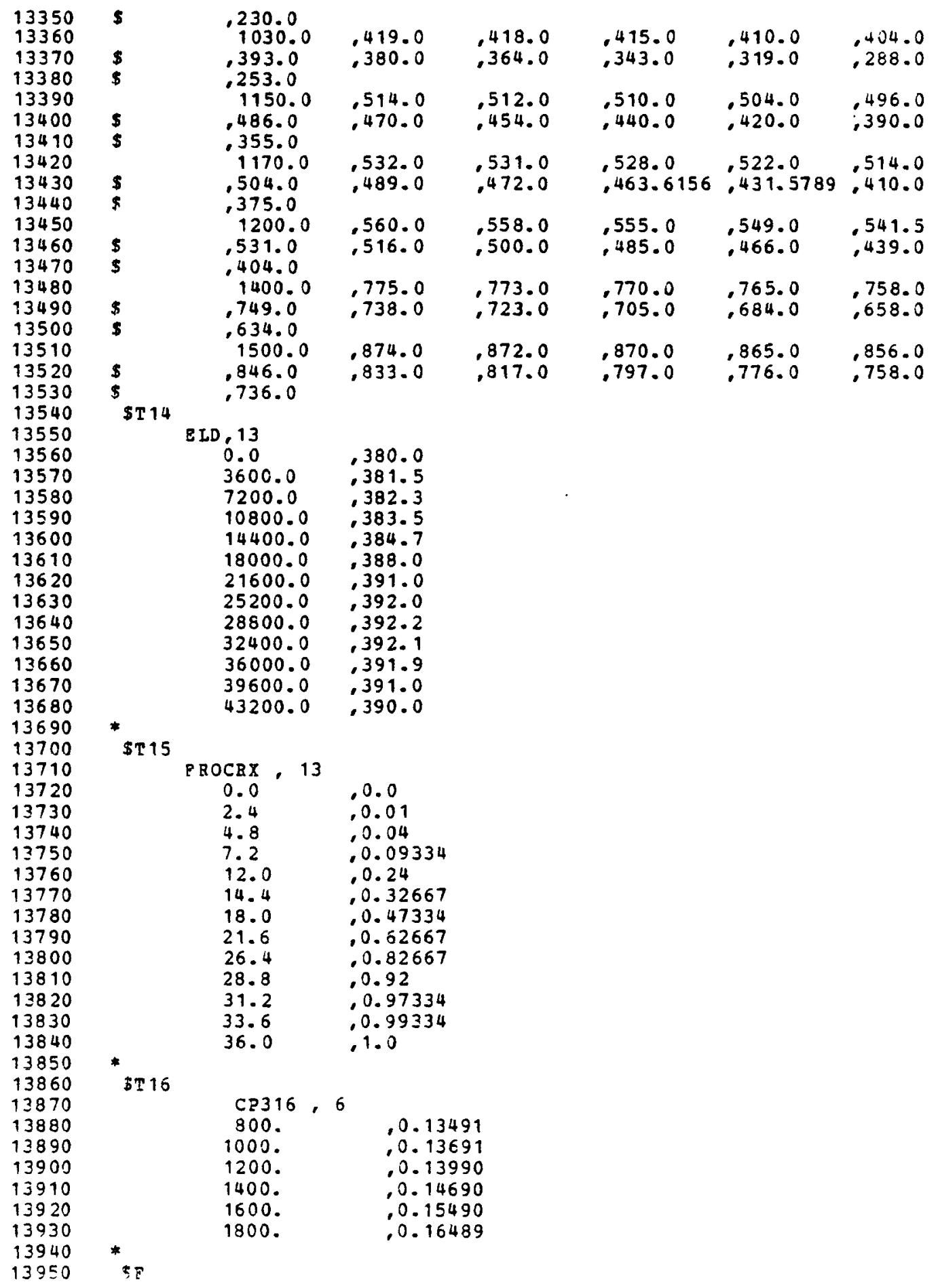




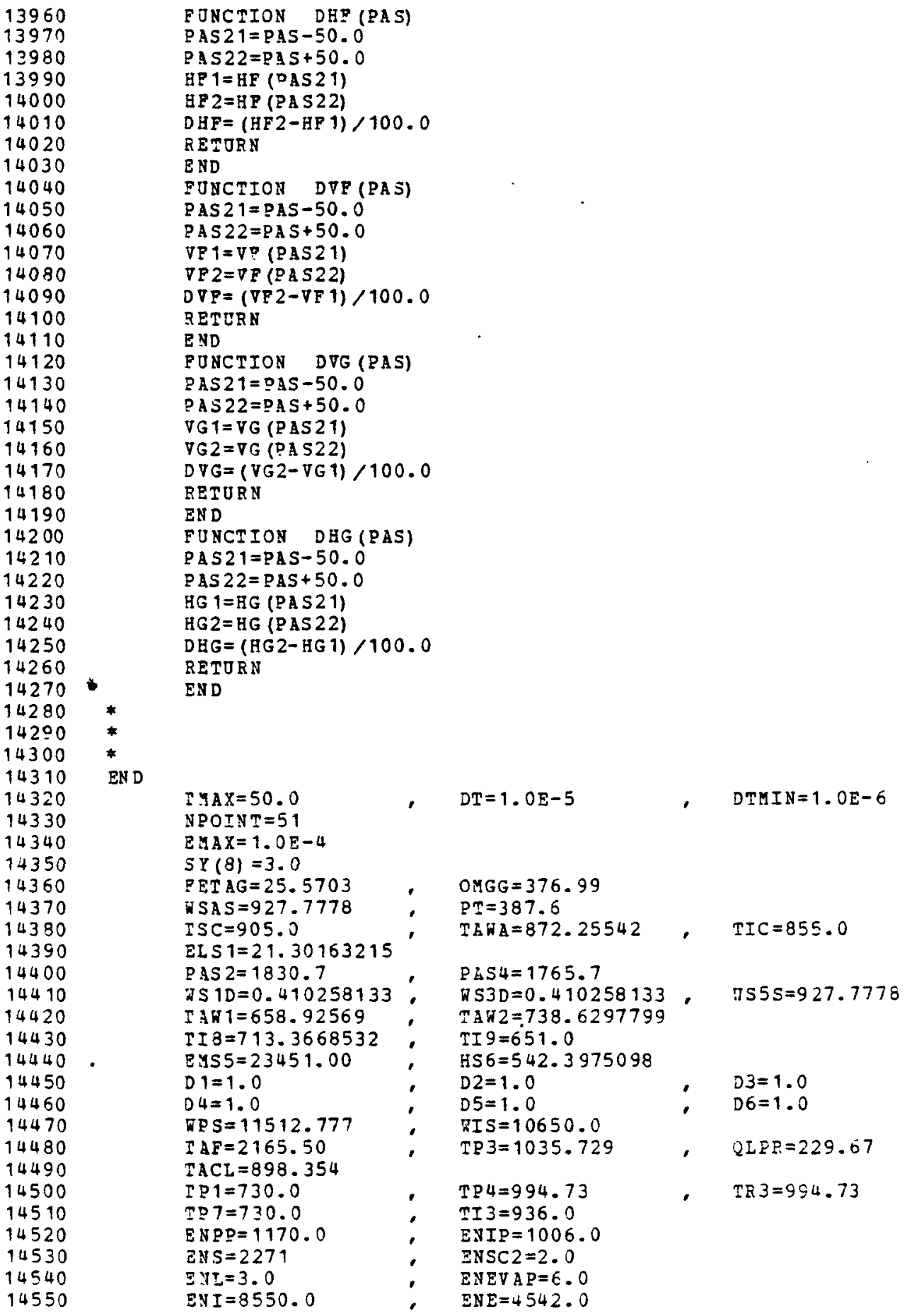




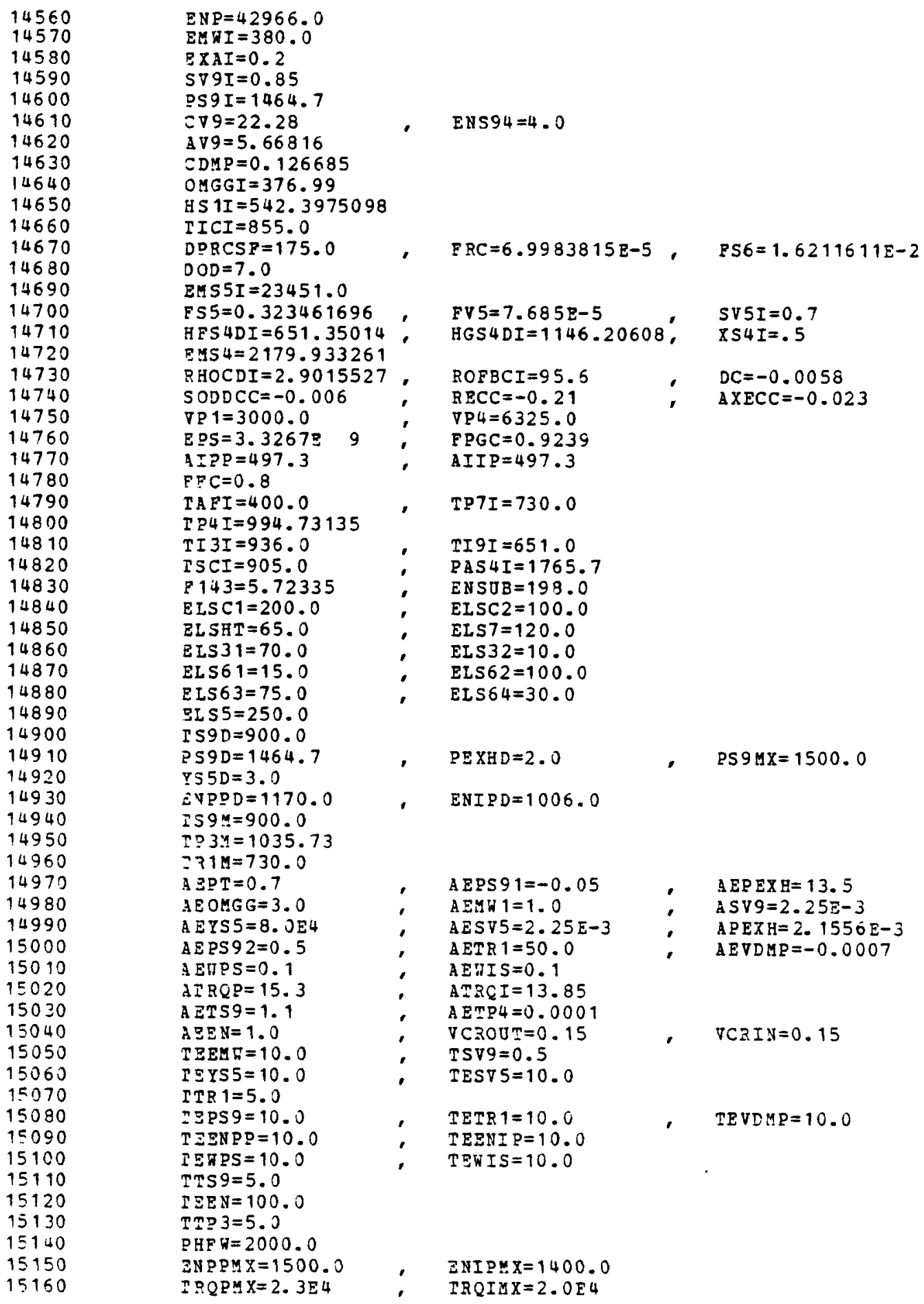




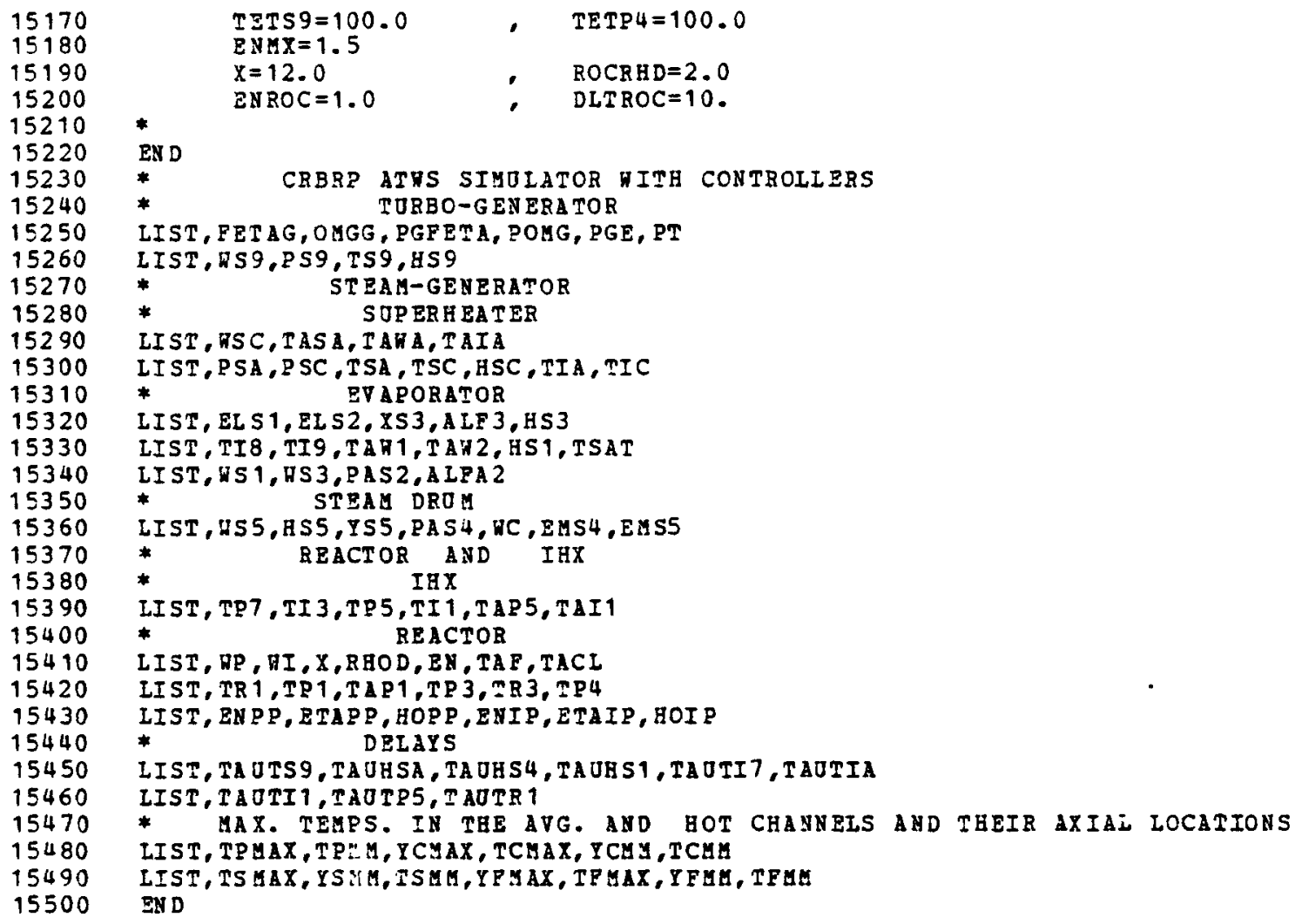




\section{APPENDIX C}

\section{Some Comments on Computer Graphics}

In addition to the capability of generating output listings such as the samples in the report, DARE $P$ contains two graphics packages (1ine printer plots and CALCOMP graphs). At our local installation, we have augmented these by interactive graphics using a Tektronix Model 4010-1 terminal in conjunction with a DEC-10 computer. Samples are shown in Figs. 2-5.

These graphs are generated on the Tektronix screen using interactive FORTRAN programs that call the Tektronix Advanced Graphics subroutines. The DARE $P$ output listings are sent over a computer link from the CYBER-175 to the DEC-10, where they appear as disk files. These files are read by the interactive graphics programs, and graphs are plotted in response to keyboard commands.

Options include: number and size of graphs, line style, number of grid lines, placement of labels, and the ability to select a portion of any graph for plotting on an expanded scale. This capability is very useful in the interpretation of large system simulations. 
POSTSCRIPT

Since this manual was prepared, an error was discovered in the reactor thermodynamic equations.

The error is in the heat transfer coefficients for heat transfer from fuel to cladding and cladding to coolant. To correct this, the following lines should be changed.

$$
\begin{array}{ll}
230-270 & \text { Replace } 2.4215565 \text { with } 2.4524460 \text { wherever it appears. } \\
7800 & \text { HR1 }=9.4247778 * \mathrm{ENP} /(0.125 / \mathrm{AKF}+60.00024 / \mathrm{HDG} \pm .0027891) \\
7810 & \mathrm{HR} 2=9.4247778 * \mathrm{ENP} /(52.173914 / \mathrm{HC}+.0024258) \\
14480 & \text { TAF }=\underline{2202.67}, \mathrm{TP} 3=1035.729, \text { OLPR }=229.67 \\
14490 & \text { TACL }=\underline{916.8293}
\end{array}
$$

The underlines indicate the portions that have alterations or additions. The resulting changes in the output are very small. The peak value of TAF changes from 2237.44 to 2275.56 ; the increase in TAF above its initial value changes from 71.94 to 72.89 . The peak value of TACL changes from 902.387 to 921.603 ; the increase in TACL above its initial value changes from 4.033 to 4.774 . The peaks are not shifted in time. The reactivity RHOD changes sign at the right time, and typical values are, at $t=50,-8.23877 E-4$ (old) and $-8.17164 E-4$ (new). Most of the other variables are affected only in the sixth significant figure (if at a11).

The BRENDA models are under continuing review and evalution. A file of changes is being maintained, and is available from the authors on request. 